DOE/ET/20424-T7

\title{
RESEARCH EXPERIMENTS DETAILED DESIGN REVIEW
}




\section{DISCLAIMER}

This report was prepared as an account of work sponsored by an agency of the United States Government. Neither the United States Government nor any agency Thereof, nor any of their employees, makes any warranty, express or implied, or assumes any legal liability or responsibility for the accuracy, completeness, or usefulness of any information, apparatus, product, or process disclosed, or represents that its use would not infringe privately owned rights. Reference herein to any specific commercial product, process, or service by trade name, trademark, manufacturer, or otherwise does not necessarily constitute or imply its endorsement, recommendation, or favoring by the United States Government or any agency thereof. The views and opinions of authors expressed herein do not necessarily state or reflect those of the United States Government or any agency thereof. 


\section{DISCLAIMER}

Portions of this document may be illegible in electronic image products. Images are produced from the best available original document. 


\section{The following pages are an exact representation of what is in the original document folder.}




\section{Collector Subsystem Project Task Logic Summary}

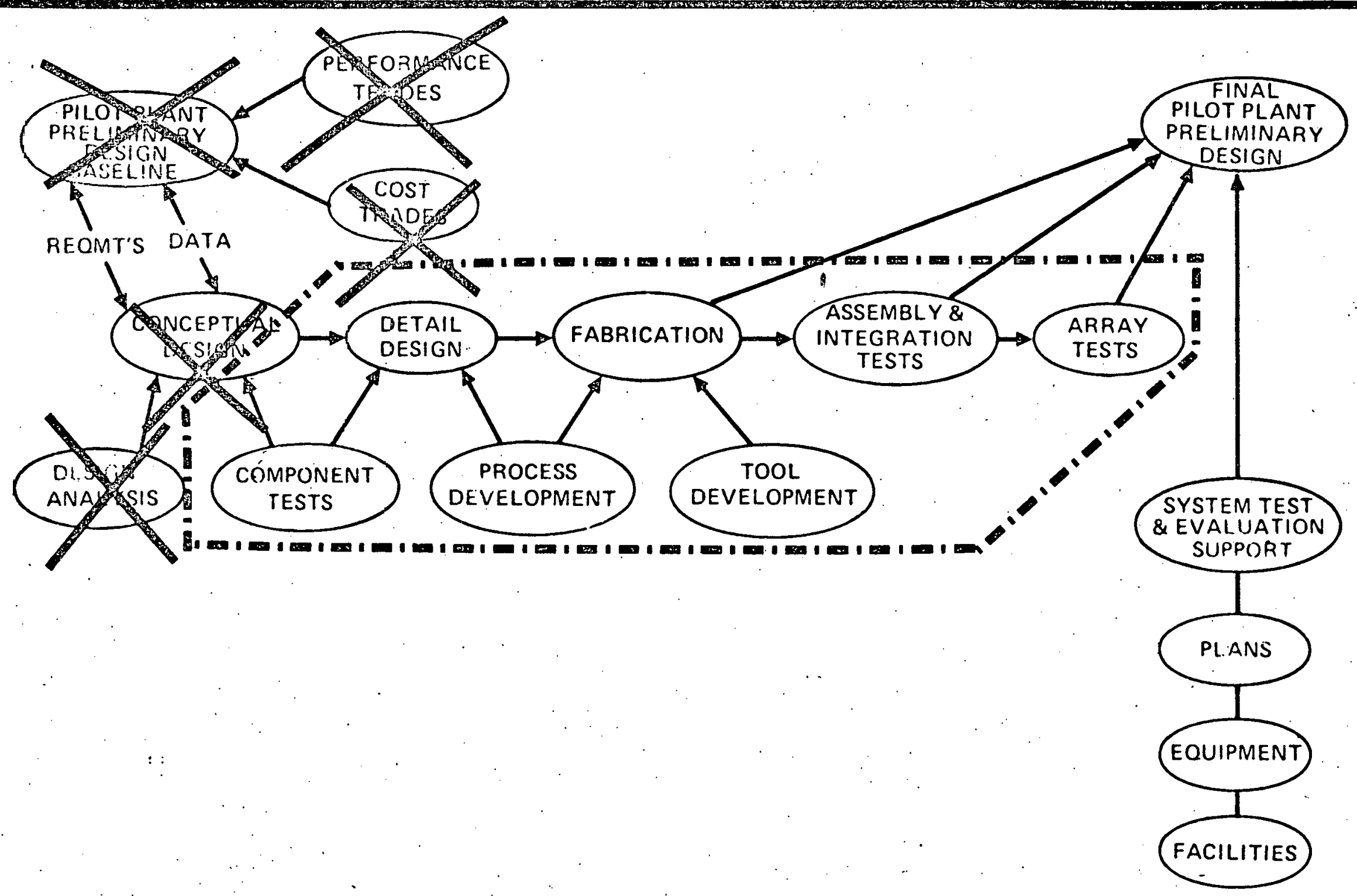




\section{AGENDA FOR DDR PRESENTATION}

February 18, 1976

9:00 AM

$9: 30$

$10: 00$

$11: 45$

12:45 PM

$1: 45$

$2: 45$

$3: 20$

$4: 31]$
PROJECT REVIEW

TRANSPARENT ENCLOSURE ASSEMBLY DESIGN

Requirements

Configuration

Materials

COFFEE BREAK

Air Supply

Safety

Structural Design

LUNCH

LAB TOUR - FOCUSSING REFLECTOR

REFLECTIVE ASSEMBLY DESIGN

Requirements

Configuration

Materials

Safety

Structural Design

TRANSPARENT ENCLOSURE \& REFLECTIVE ASSEMBLY MANUFACTURING

ADJOURN 


\section{AGENDA FOR DDR PRESENTATION}

February 19, 1976

9:00 AM

$10: 00$

$10: 30$

$10: 45$

$11: 55$

1:00 PM

$1: 45$

$1: 55$

4:00
DRIVE \& CONTROL ASSEMBLY DESIGN

Requirements

Configuration

Components

Safety

Software

COFFEE BREAK

RESEARCH EXPERIMENT TEST RESULTS

LUNCH

ASSEMBLY, INTEGRATION \& ARRAY TEST PLANS

PLANS FOR NEXT QUARTER

REVIEW OF ACTION ITEMS \& SUMMARY OF DISCUSSION

ADJOURN 
Solar C ctor Subsystem

Total Program
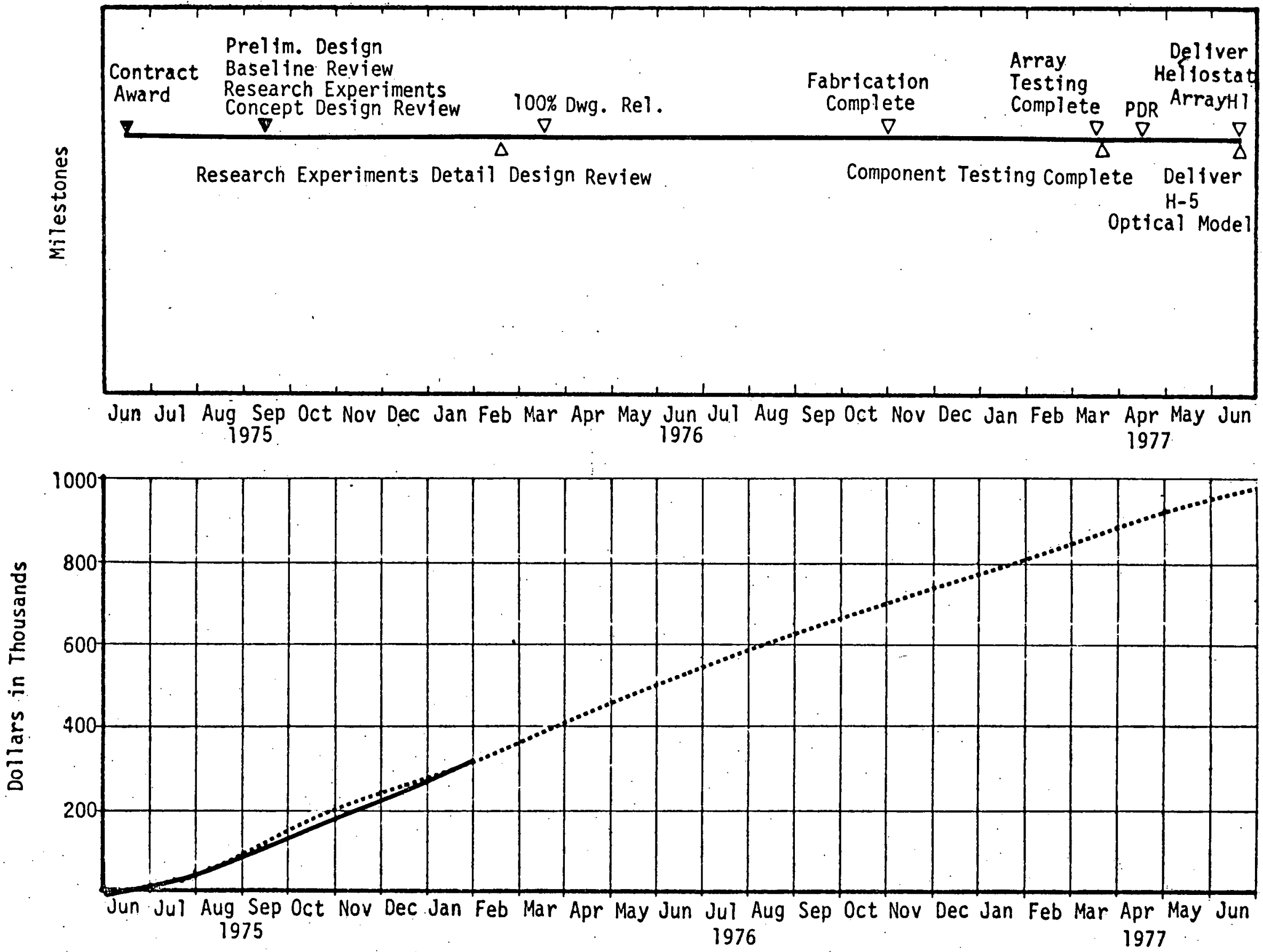
Solar Collector Subsystem

Total Program

\begin{tabular}{|c|c|c|c|c|c|c|c|c|c|c|c|c|c|c|c|c|c|c|c|c|c|c|c|c|c|}
\hline Budget $\$$ & 12 & 36 & 49 & 66 & 48 & 39 & 29 & 42 & 42 & 57 & 47 & 43 & 50 & 39 & 37 & 36 & 37 & 38 & 37 & 33 & 34 & 45 & 43 & 29 & 10 \\
\hline Actual $\$$ & 12 & 38 & 47 & 50 & 45 & 33 & 44 & 59 & & & & & & & & & & & & & & & & & \\
\hline Cum $\$$ & 12 & 50 & 97 & 147 & 192 & 225 & 269 & 328 & & & & & & & & & & & & & & & & & \\
\hline Eudget $M$ & 1.8 & 11.8 & 14.7 & 13.6 & $7 i .5$ & 10.7 & 9.0 & 8.2 & 7.5 & 8.2 & 7.8 & 7.6 & 7.6 & 9.1 & 8.4 & 7.8 & 8.6 & 8.8 & 8.3 & 7.1 & 7.2 & 7.8 & 8.3 & 7.3 & 2.0 \\
\hline Actual M & 1.8 & 11.6 & 13.1 & 11.2 & 12.1 & 8.7 & 12.91 & 12.6 & & & & & & & & & & & & & & & & & \\
\hline
\end{tabular}
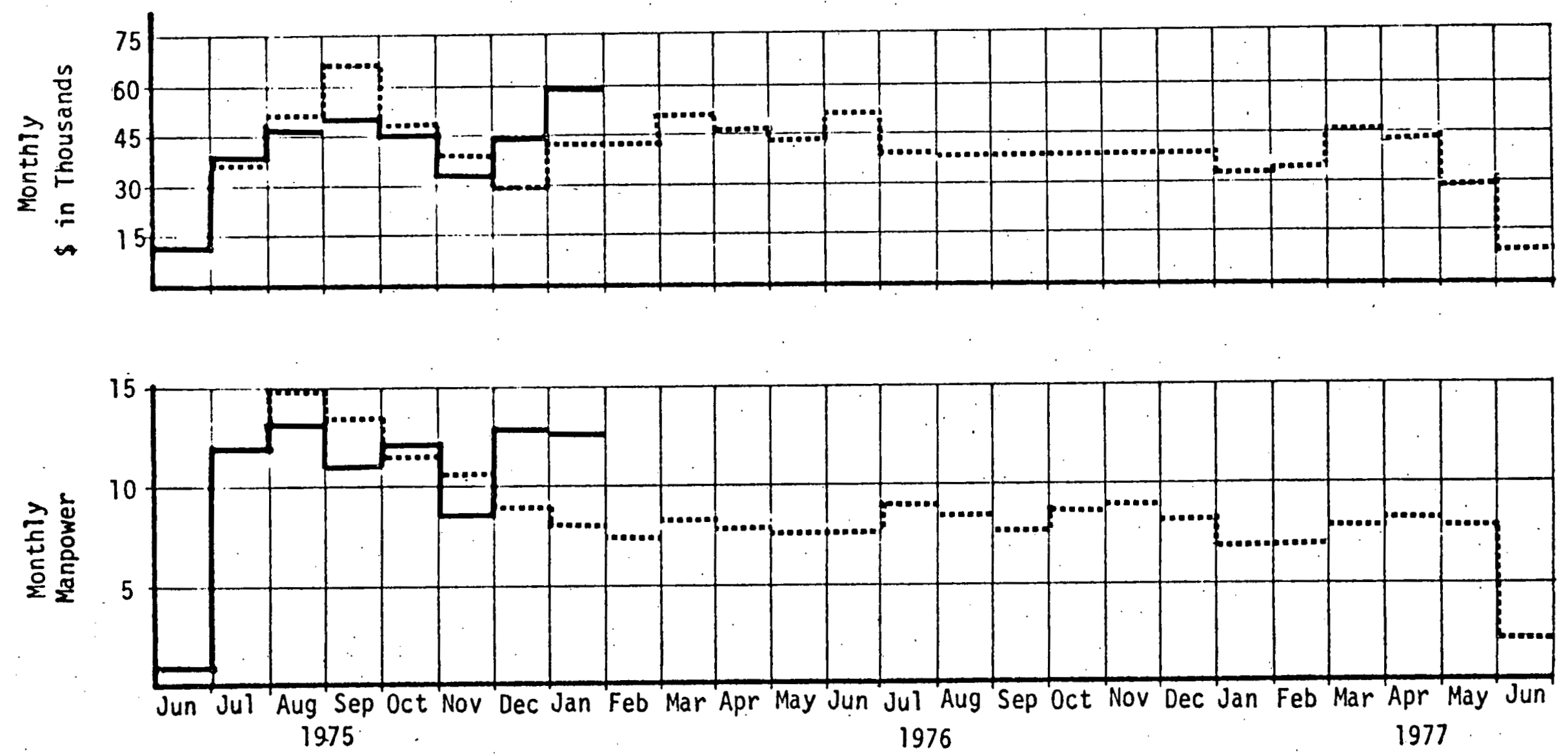

1. 
Solar C ctor Subsystem

SC 210 Collector Subsystem Baseline
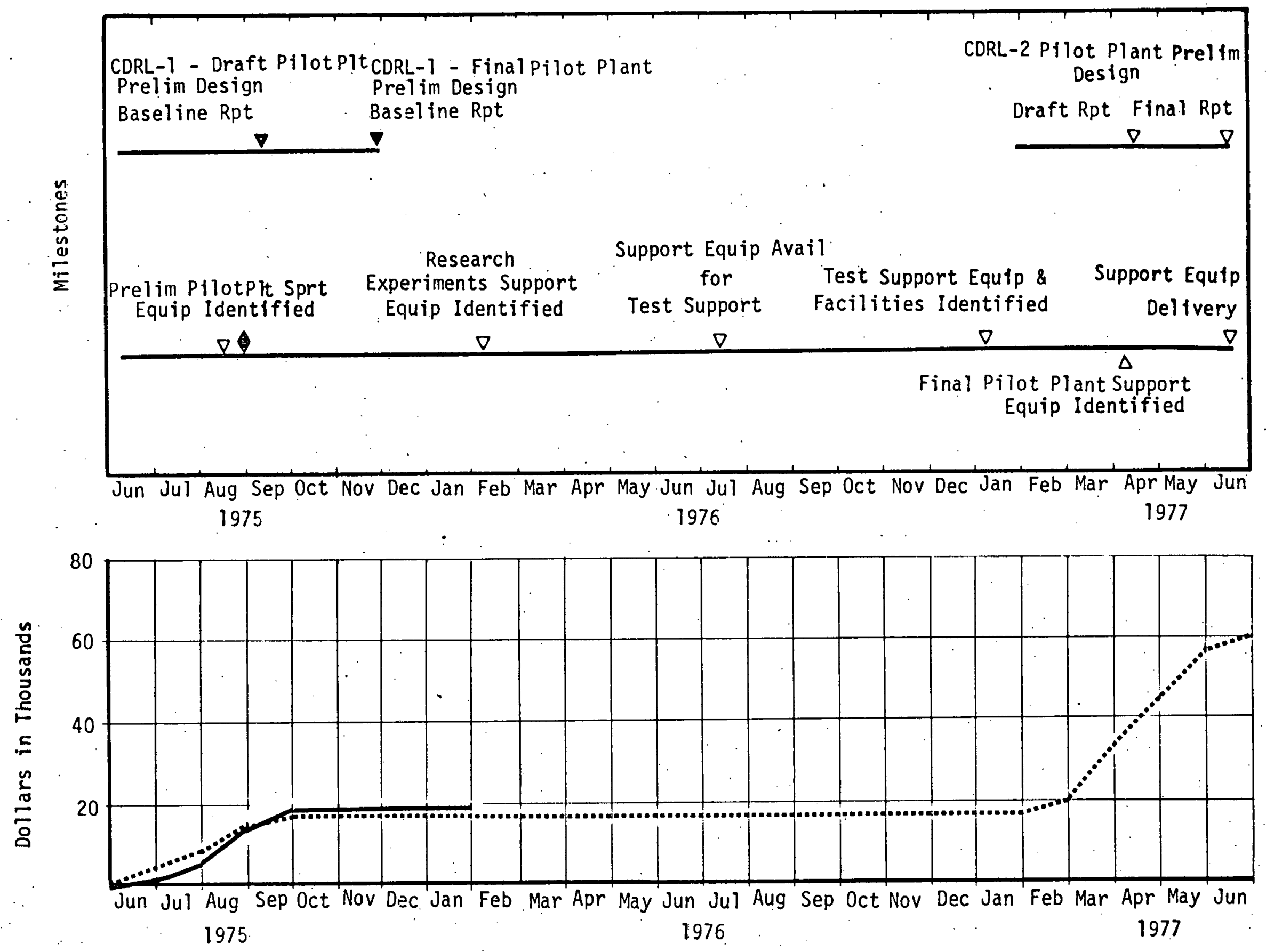
Soiar iollector Subsystem

SC 220 Collector Subsystem Research Experiments

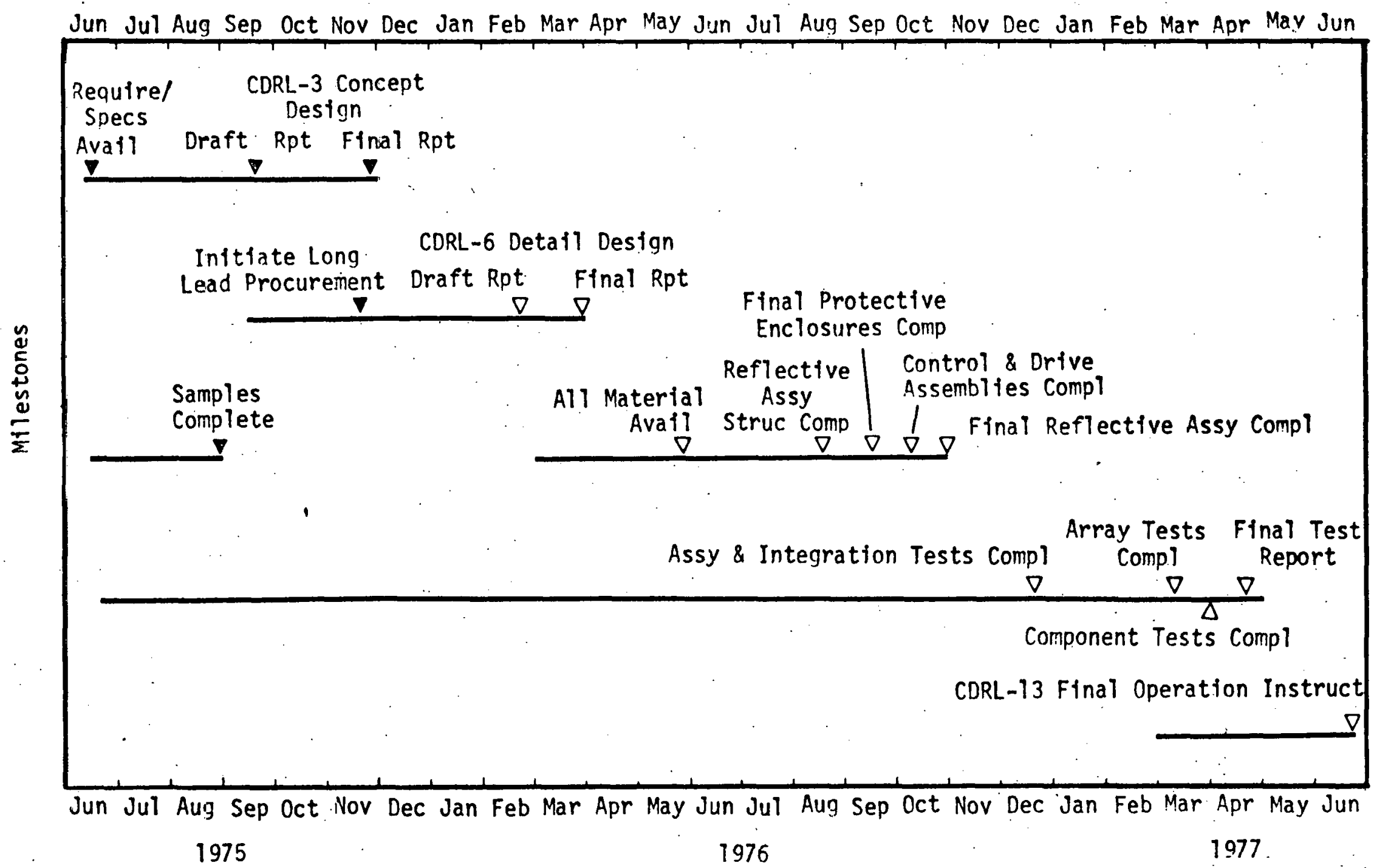


Solar Collector

SC 220 Collector Subsystem Research Experiments

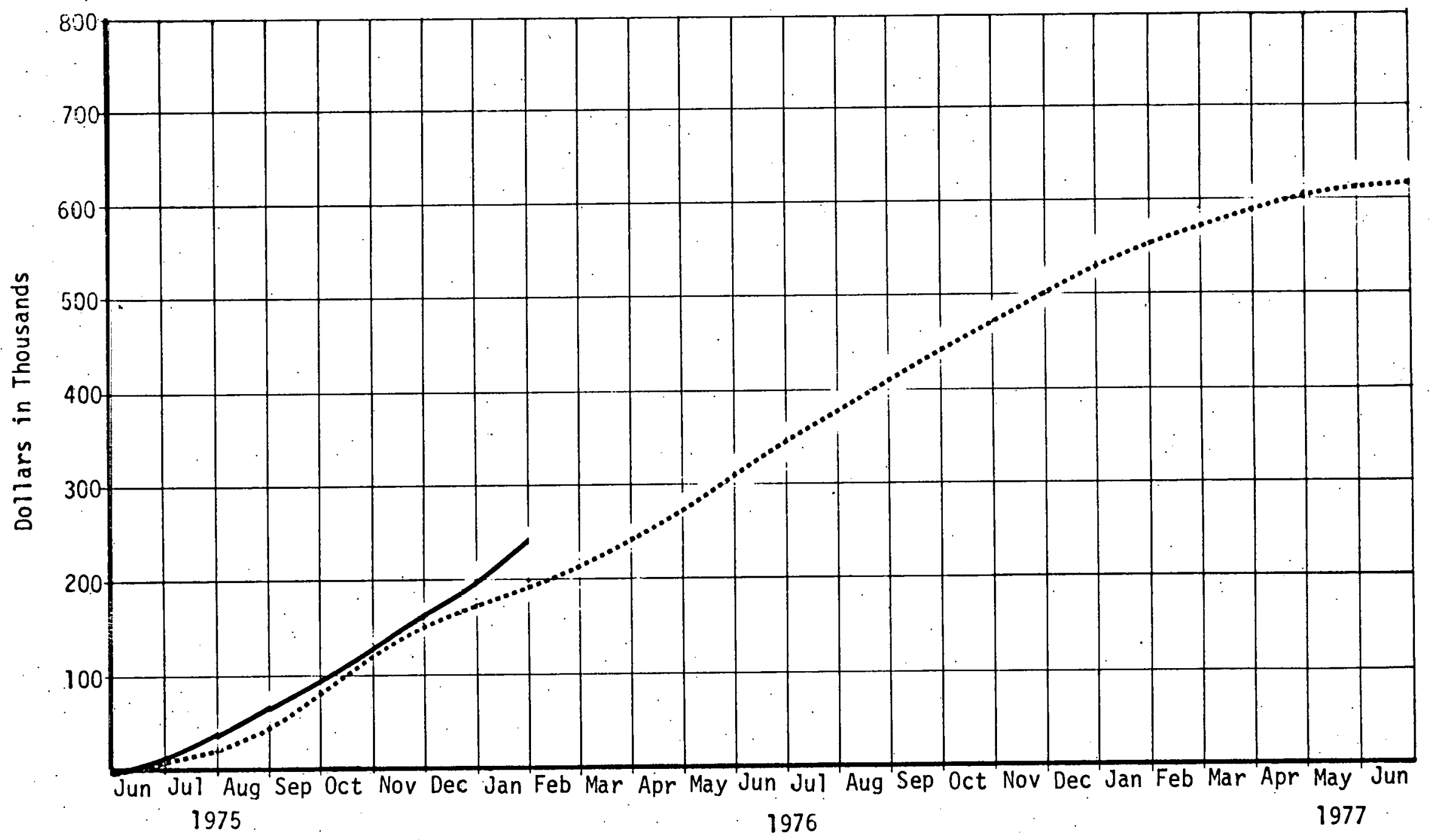


Solar Collector Subsystem

SC 220 Collector Subsystem Research Experiments
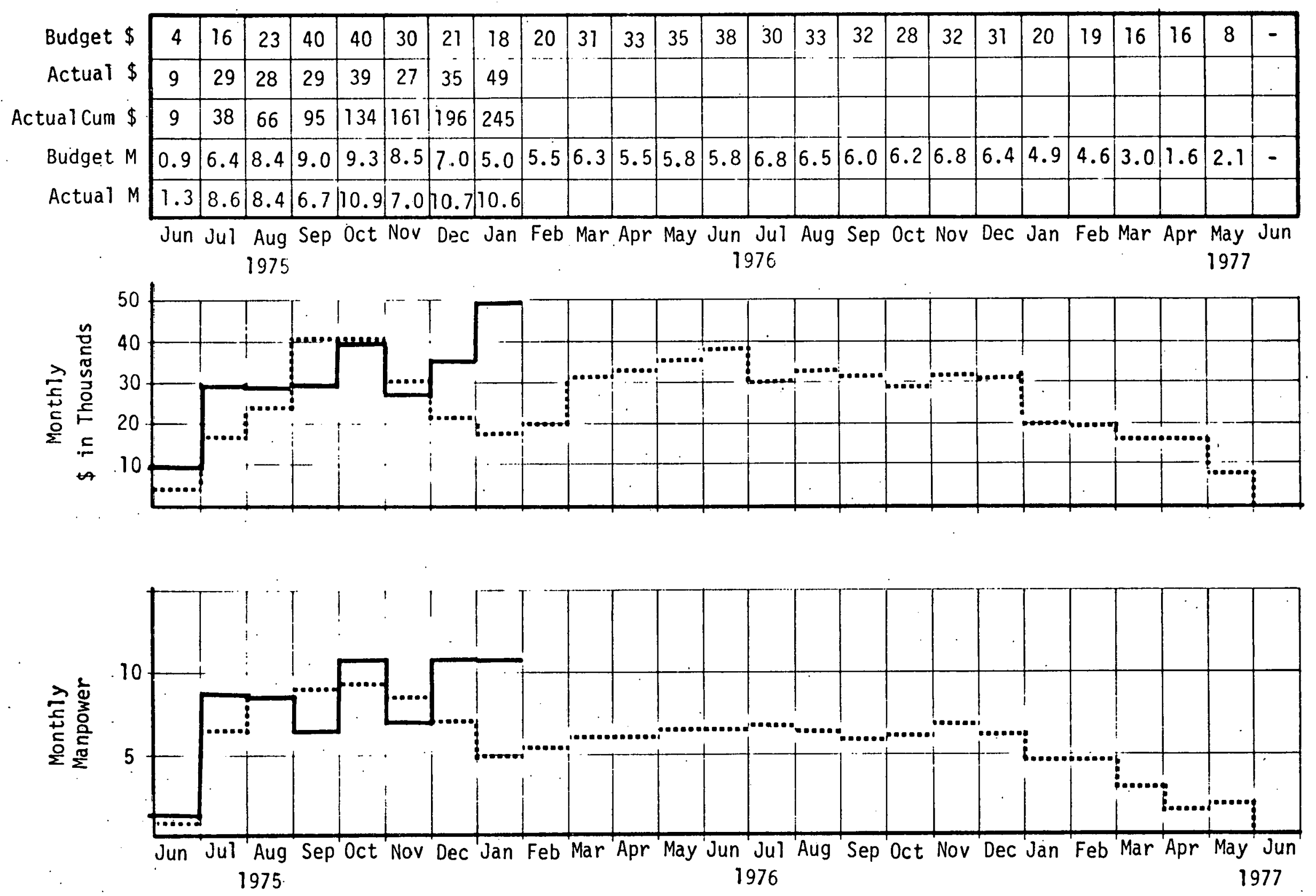
Solar C . . ctor Subsystem

SC. 060 System/Project Mgmê
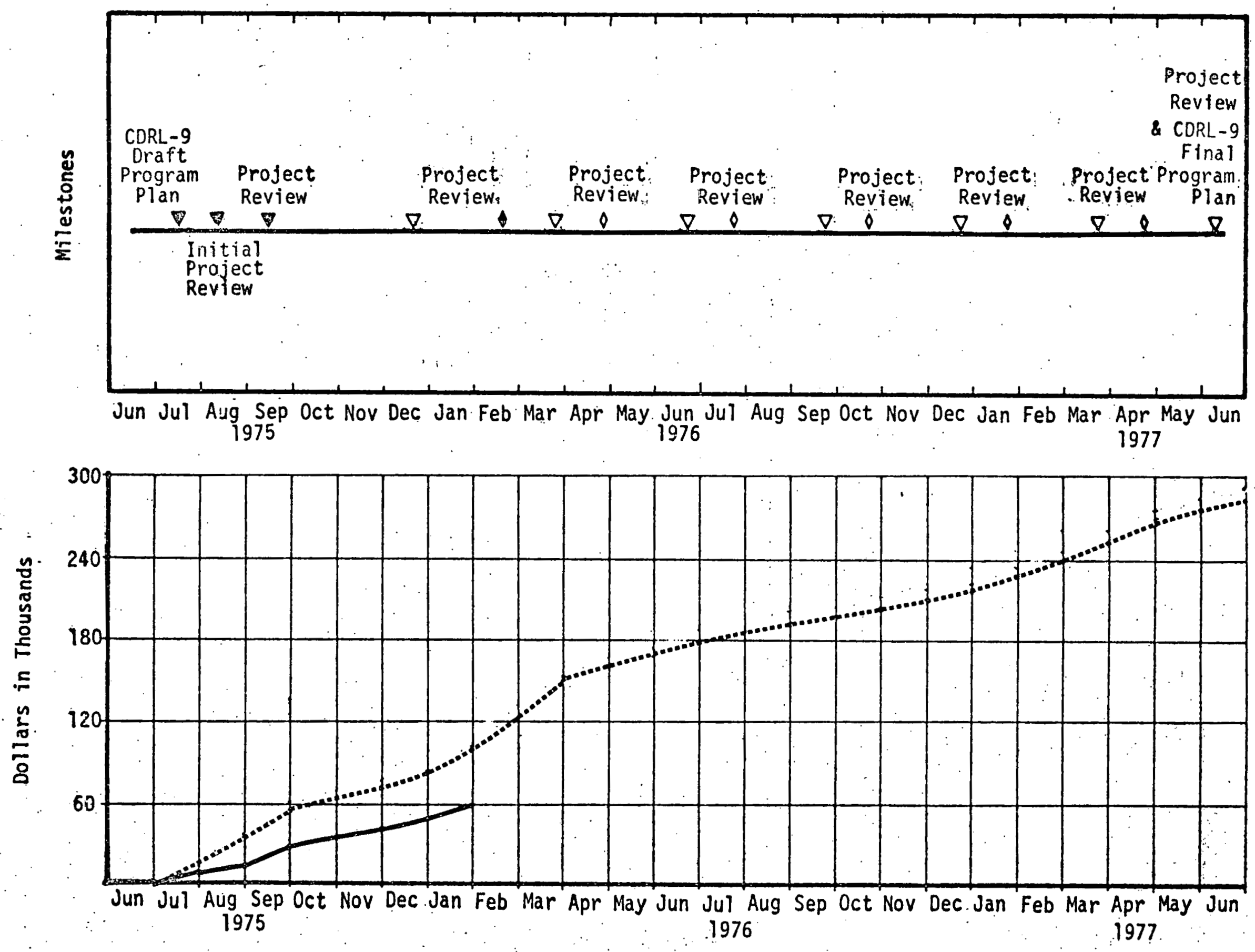

I. 
Solar C: ctor Subsystem

SC 070 Data
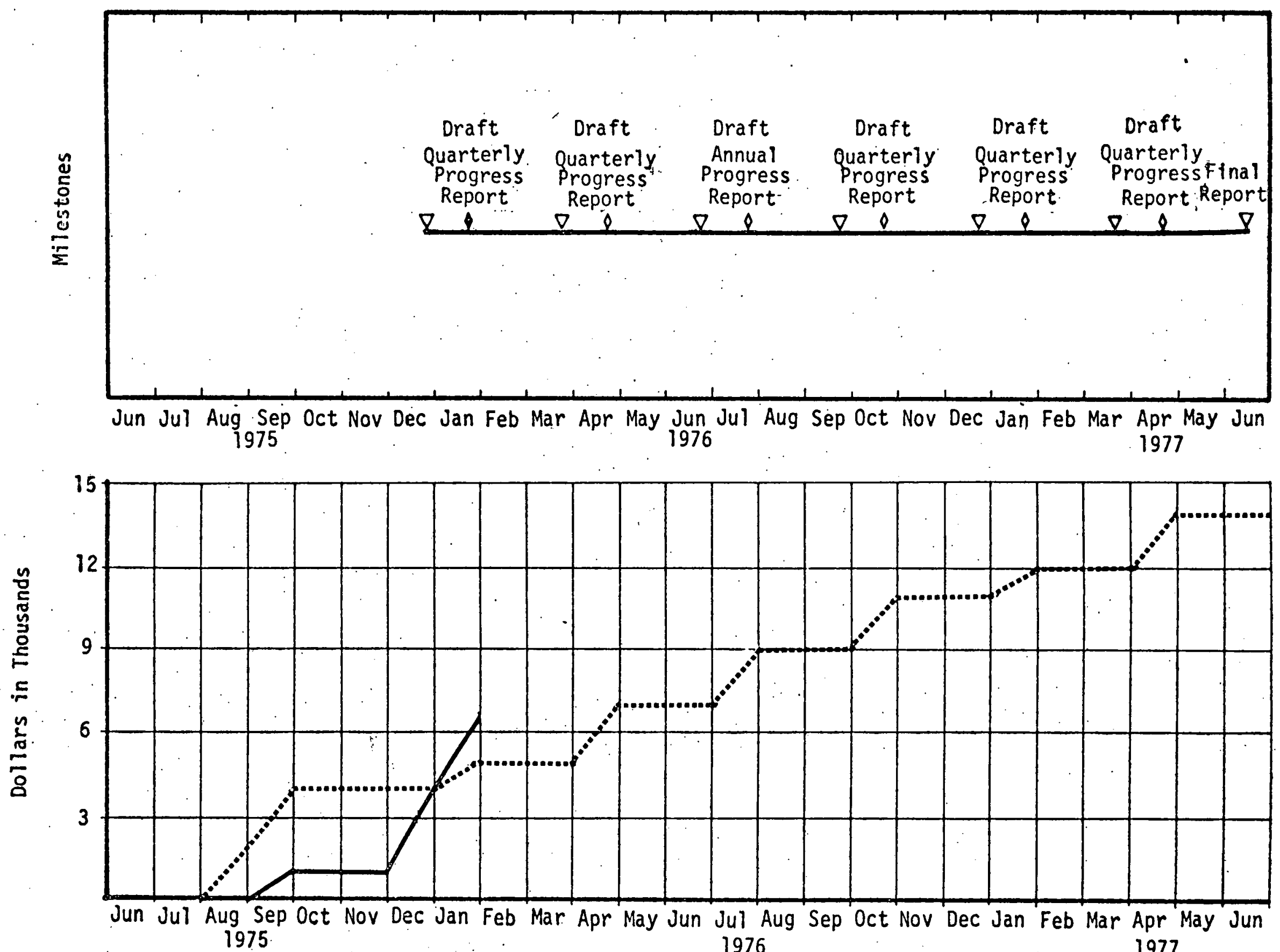


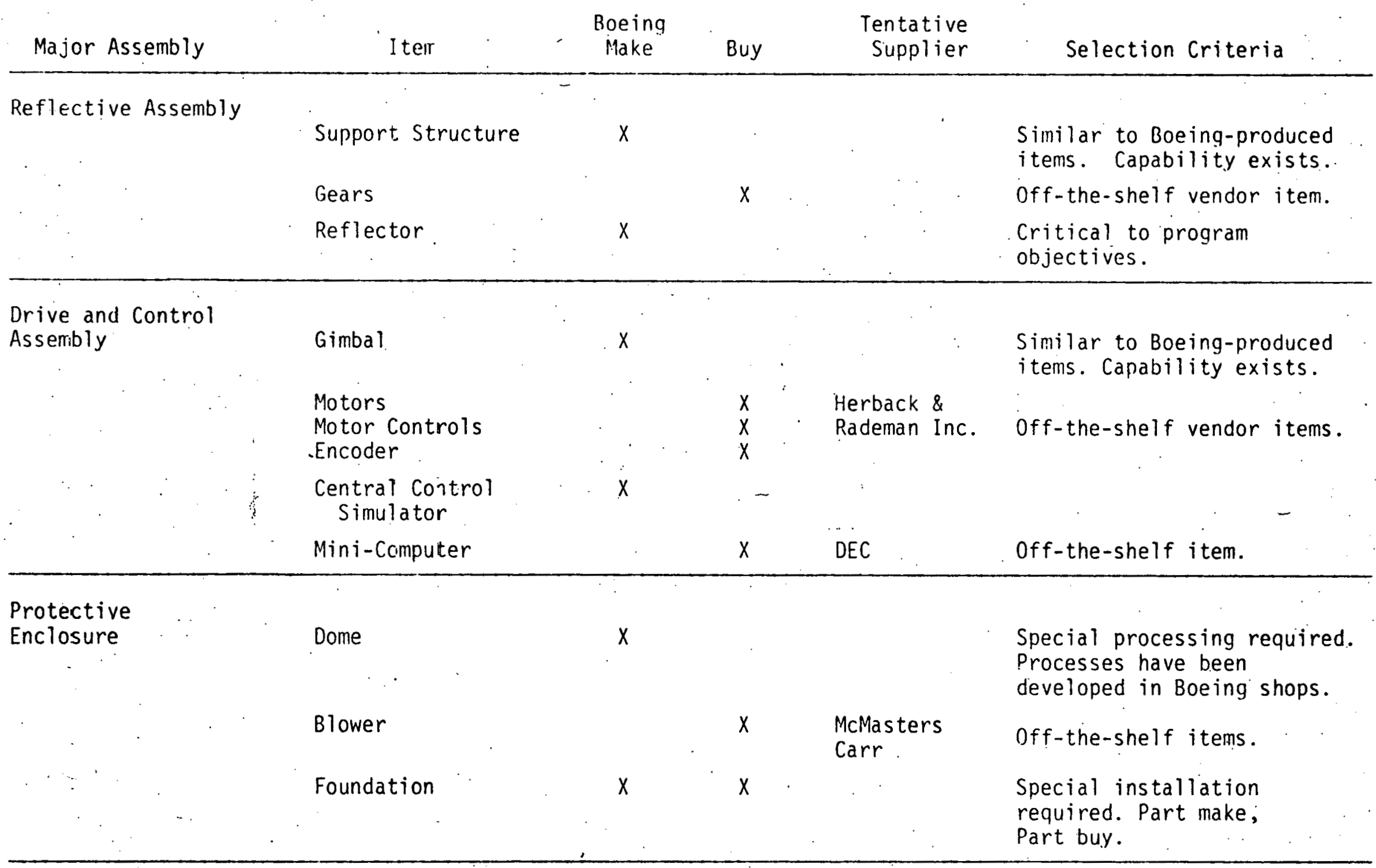

Table 4-1 - Make/Buy Equipment List 
MAJOR ITEM F UREMENT SCHEDULE

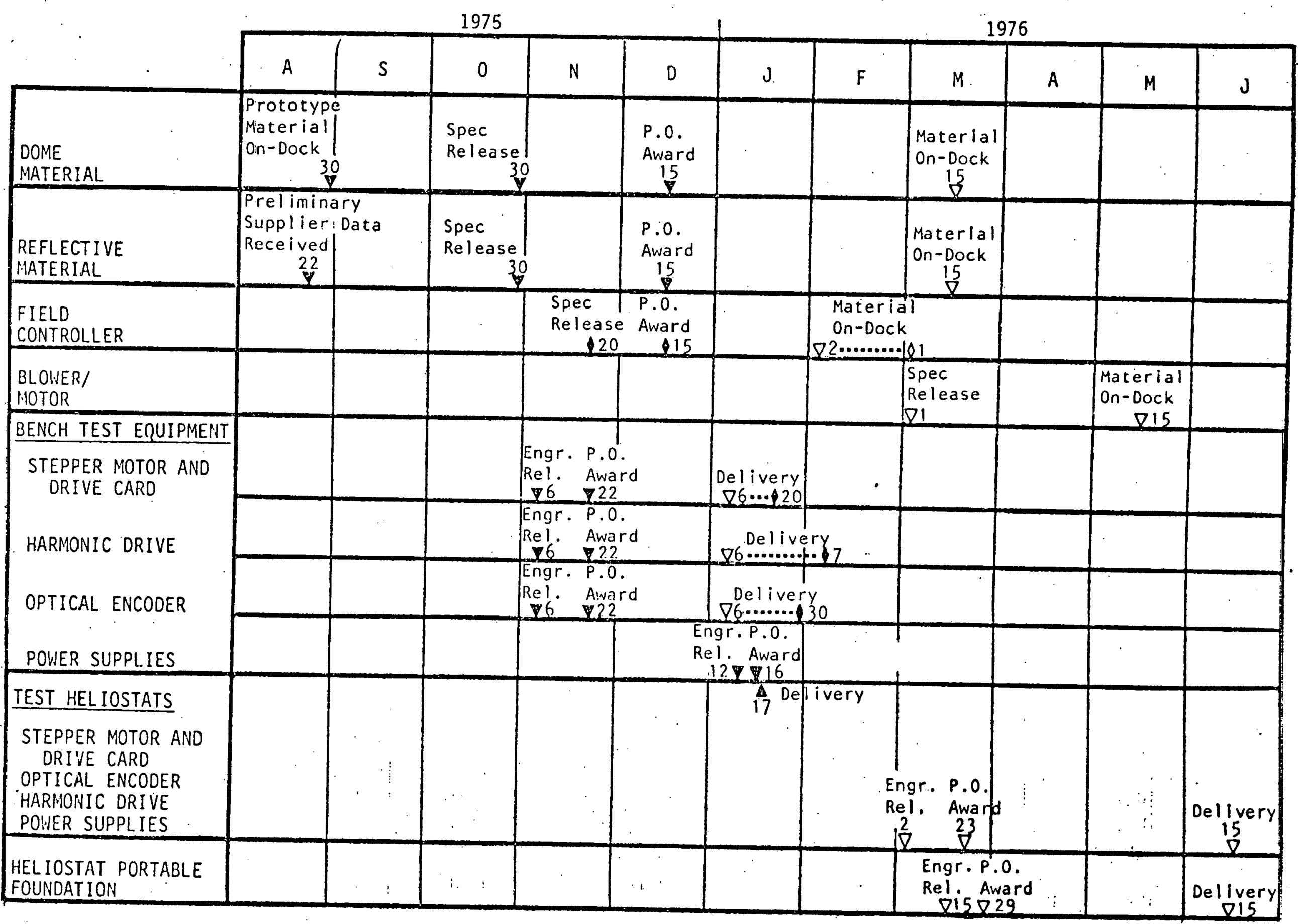


test schedule for the least contract cost are shown in Table 4-2. The spares selection was based on an analysis of the potential need, cost and refurbishlent recycle times of the array equipments.

\section{Spares List}

Spare Qty. Justification

\begin{tabular}{|c|c|c|}
\hline Spare & Qty. & Justification \\
\hline Transparent Enclosure & 2 & $\begin{array}{l}\text { Replacements for test to } \\
\text { avoid potential schedule } \\
\text { slippage }\end{array}$ \\
\hline Reflective-Membanes & $z$ & $\begin{array}{l}\text { Replacements for test-to } \\
\text { aroid-ontial-sehedute } \\
\text { stippage }\end{array}$ \\
\hline Drive Assembly & 1 & $\begin{array}{l}\text { Motor, Harmonic Dri } \\
\text { Replacement } t_{\lambda} \text { for test to avoid } \\
\text { potential schedule slippage }\end{array}$ \\
\hline Reftector-strueture & -7 & $\begin{array}{l}\text { Replacement-fop-test-to-avoid } \\
\text { potential schedule-stippage }\end{array}$ \\
\hline Reflective Assembly & 1 & $\begin{array}{l}\text { Replacement for test to avoid } \\
\text { potential schedule slippage }\end{array}$ \\
\hline
\end{tabular}

Table 4-2 
Collector Subsystem Critical Performance Parameters

\begin{tabular}{|l|c|c|c|}
\hline \multicolumn{1}{|c|}{ Key Parameters } & Spec. & Goal & $\begin{array}{l}\text { Test (T) or } \\
\text { analysis (A) status }\end{array}$ \\
\hline - Specular reflectance & $85 \%$ & $87 \%$ & $85-89(\mathrm{~T})$ \\
- Specular transmittance of dome & $86 \%$ & $90 \%$ & $85.89(\mathrm{~T})$ \\
- Orientation accuracy & $\begin{array}{c} \pm .05^{\circ} \\
\text { (RSS) }\end{array}$ & $\begin{array}{c} \pm .05^{\circ} \\
\text { (RSS) }\end{array}$ & $\begin{array}{c} \pm .05^{\circ}-.16^{\circ}(\mathrm{A}) \\
\text { (RSS) }\end{array}$ \\
\hline
\end{tabular}


Collector Subsystem

\section{Heliostat Assembly for Research Experiments}

HELIOSTAT FEATURES

- TEDLAR DOME

5.18 METER DIAMETER

$0.10 \mathrm{MM}$ THICK

- ALUMINIZED MYLAR REFLECTOR

4.57 METER DIAMETER

$0.05 \mathrm{MM}$ THICK

- OPEN-LOOP AUTOMATED CONTROL MINIPROCESSOR CONTROL.

SEMI-AUTOMATED CALIBRATION

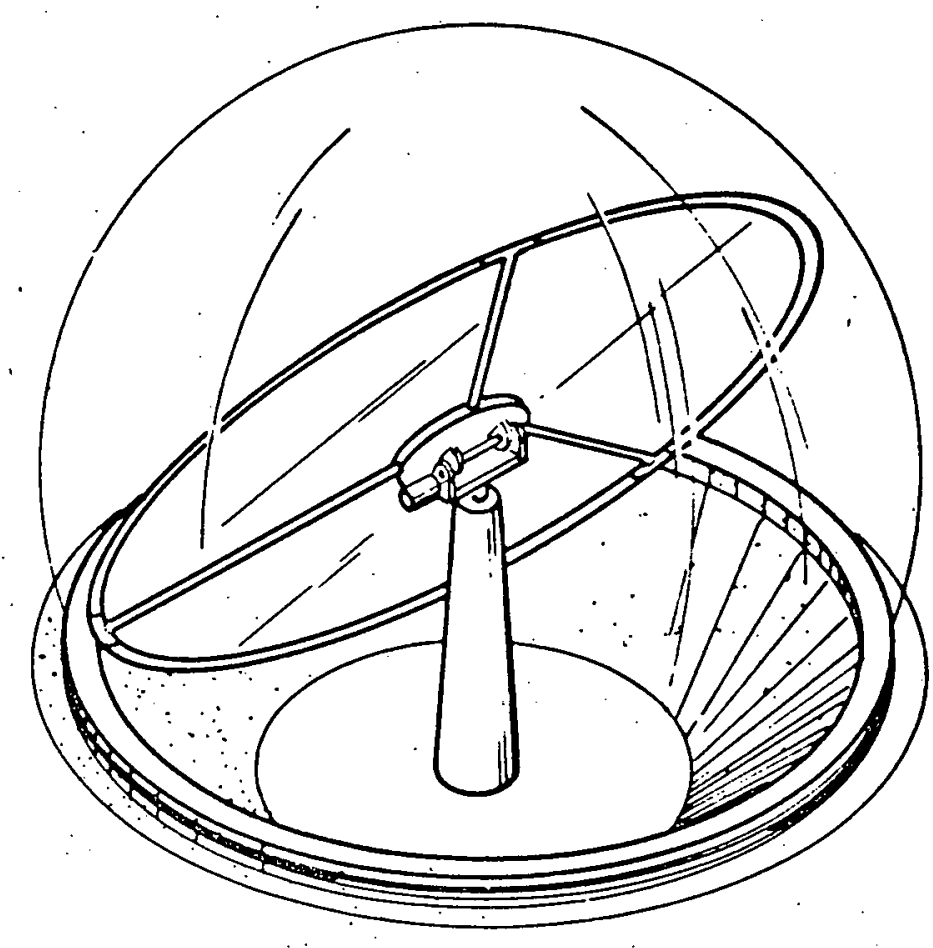




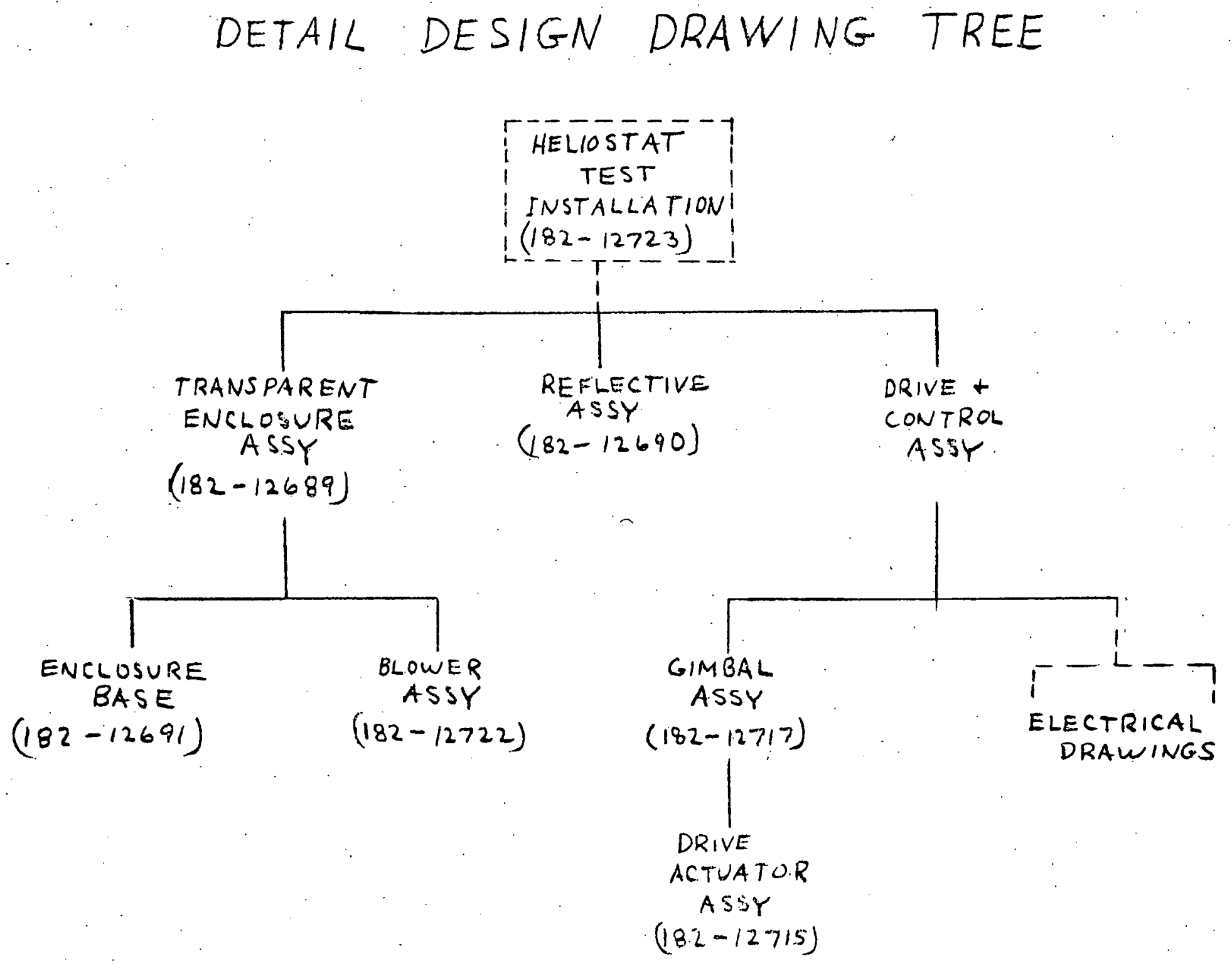




\begin{tabular}{|c|c|}
\hline INSTALLATION & $\begin{array}{l}\text { NORMAL SAFETY PROCEDURE FOR EXCAVATION \& CONCRETE HANDLING WITH } \\
\text { HEAVY EQUIPMENT. PREVENTION OF PUBLIC ACCESS. NORMAL HARD-HAT } \\
\text { SAFETY PROCEDURES AND EQUIPMENT FOR OVERHEAD CRANE. INSTALLATION OF } \\
\text { EQUIPMENT. }\end{array}$ \\
\hline ELECTRICAL & $\begin{array}{l}\text { MANUAL CONTROL STATION AT EACH HELIOSTAT WITH CAPABILITY TO BLOCK } \\
\text { CONTROL COMMANDS FROM CENTRAL. AND TO SHUT OFF ALL POWER TO HELIO- } \\
\text { STRT. PROV ISIONS IN CENTRAL CONTROL TO SHUT OFF POWER TO INDIVID- } \\
\text { UAL HELIOSTATS. }\end{array}$ \\
\hline LIGHTING & $\begin{array}{l}\text { GROUND CENTER POST. } \\
\text { GROUND ENCLOSURE HOLD DOWN SEGMENTS. } \\
\text { GROUND REFLECTOR STRUCTURE DIRECTLY TO CENTER POST }\end{array}$ \\
\hline FLOOD. & $\begin{array}{l}\text { DO NOT SITUATE FIELD IN A FLASH FLOOD AREA. } \\
\text { IF SOIL CONDITION IS CONDUCIVE TO FORMATION OF SURFACE WATER IN } \\
\text { CLOUDBURST CONDITION PROVIDE DRAINAGE IN PATHWAYS BETWEEN DOMES. }\end{array}$ \\
\hline FIRE & $\begin{array}{l}\text { PROVIDE ADEQUATE CO }{ }_{2} \text { EXTINGUISHER WITH INSTALLATION AND } \\
\text { MAINTENANCE CREWS. EACH PERSON ENTERING. AN ENCLOSURE MUST } \\
\text { HAVE PERSONAL EQUIPMENT TO CUT OUT THROUGH ENCLOSURE. }\end{array}$ \\
\hline WIND & $\begin{array}{l}\text { EACH PERSON ENTERING AN EIICLOSURE DURING HIGH WIND CONDITIONS } \\
\text { SHOULD HAVE A HARD HAT AND PERSONAL. EQUIPMENT TO CUT THROUGH } \\
\text { ENCLOSURE. }\end{array}$ \\
\hline $\begin{array}{l}\text { SOLVENT, VAPORS, } \\
\text { OXYGEN CONSUMING } \\
\text { EQUIPMENT }\end{array}$ & $\begin{array}{l}\text { THE DOME AIR PRESSURE SYSTEM !IAY NOT PROVIDE ADEQUATE AIR FOR } \\
\text { PERSONNEL. AUDITIONAL. VENT ILATION IS MANDATORY FOR PROLONGED STAYS } \\
\text { OR IF OXYGEN CONSUMING EQUIPMANT, SOLVENT OR VAPORS ARE PRESENT IN } \\
\text { ENCLOSURE. }\end{array}$ \\
\hline
\end{tabular}




\section{DESIGN REQUIREMENTS - TRANSPARENT ENCLOSURE}

SPECULAR SOLAR TRANSMITTANCE

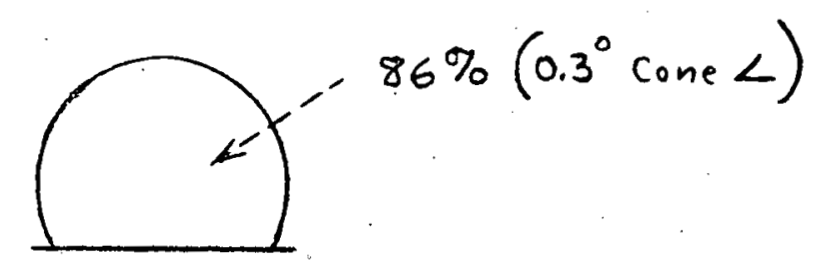

STRUCTURAL INTEGRITY

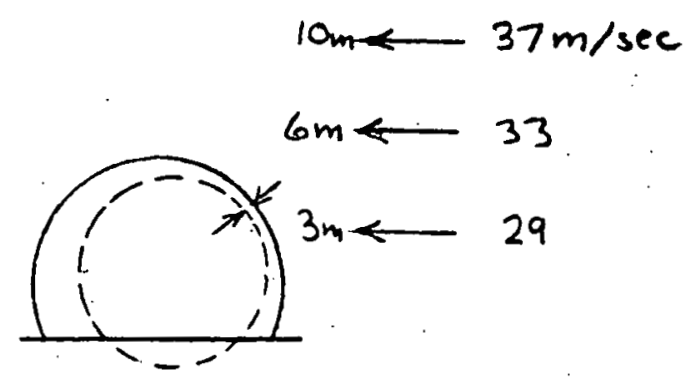

LIFE TIME

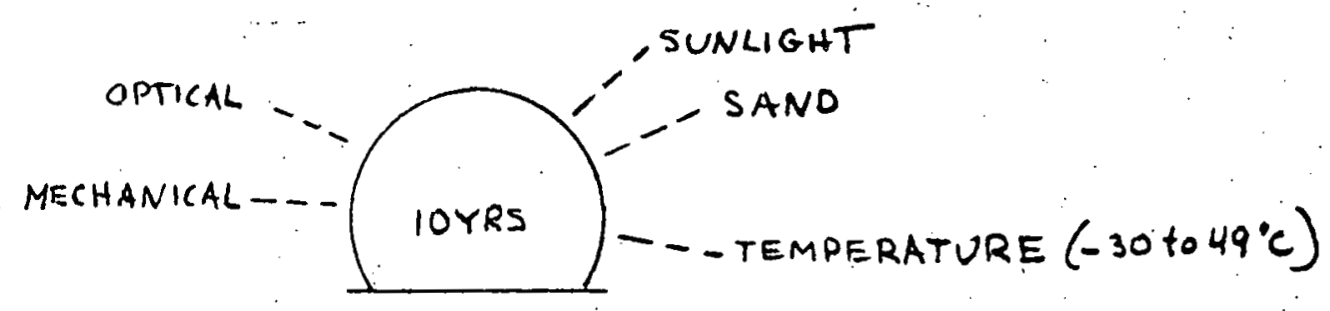

$\cos T$

$\$$ 


\section{Enclosure and Base Design for Research Experiments}

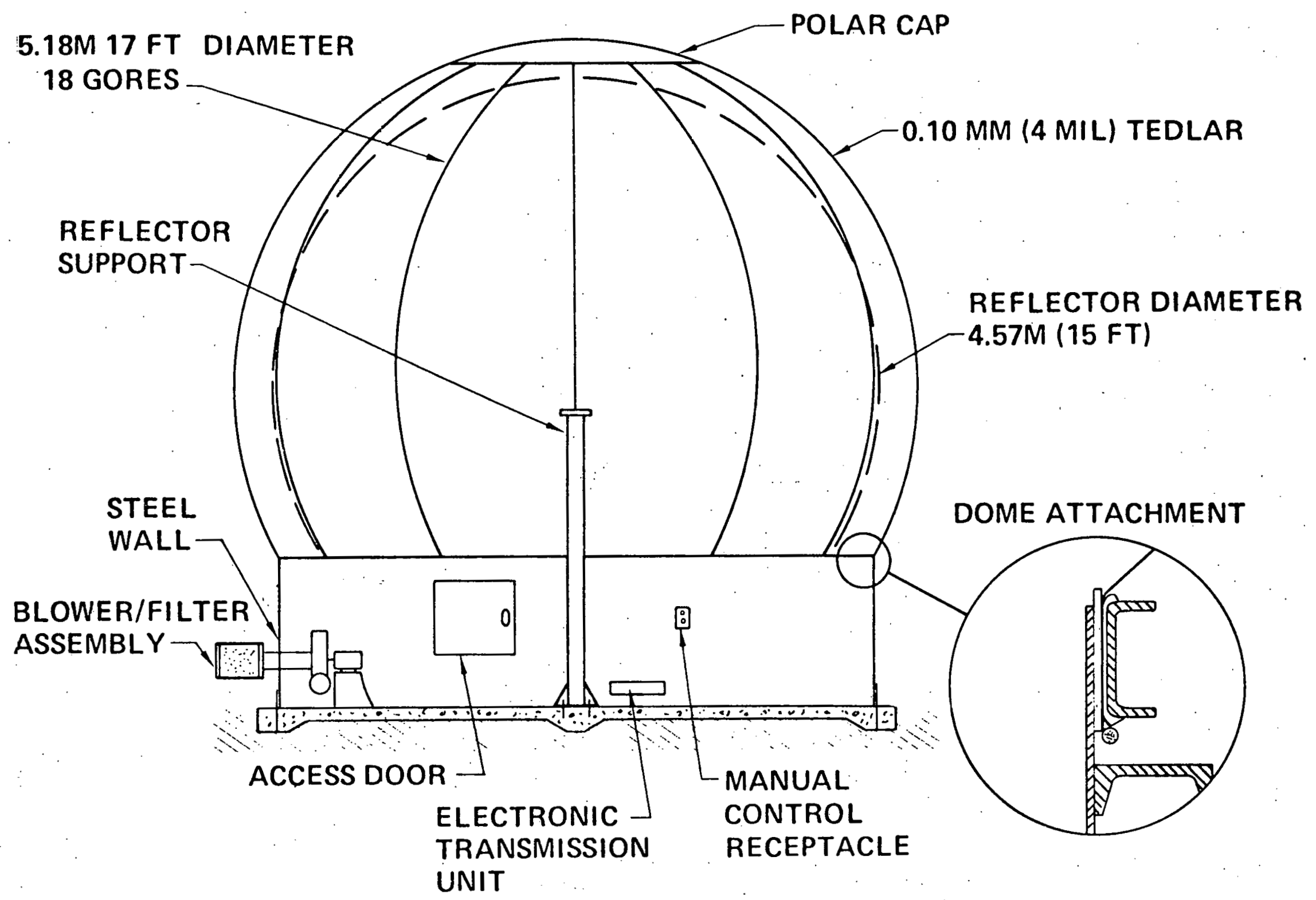




\section{Transparent Enclosure Air Supply Design Data}

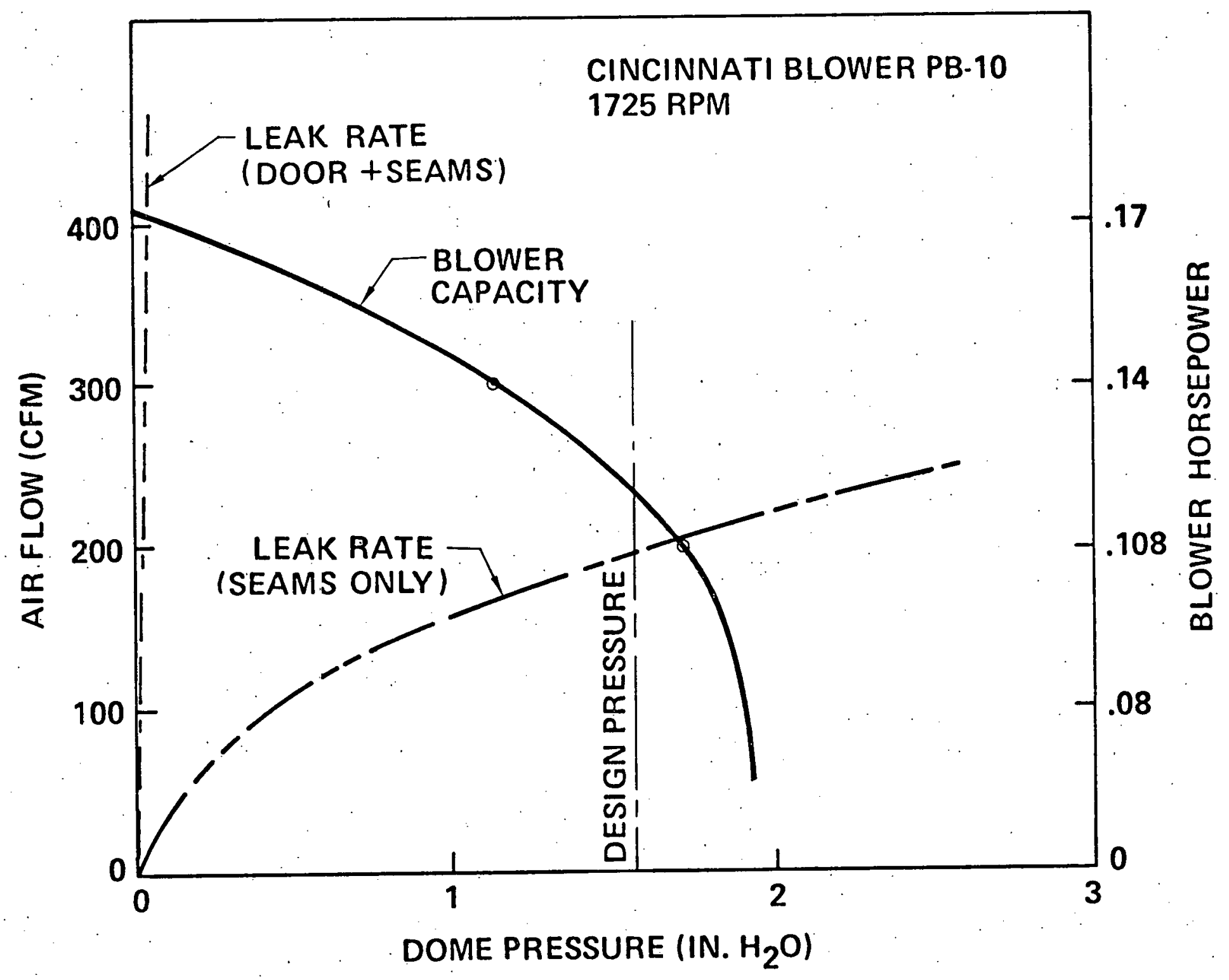




\section{DOME PRESSURE REQUIREMENTS}

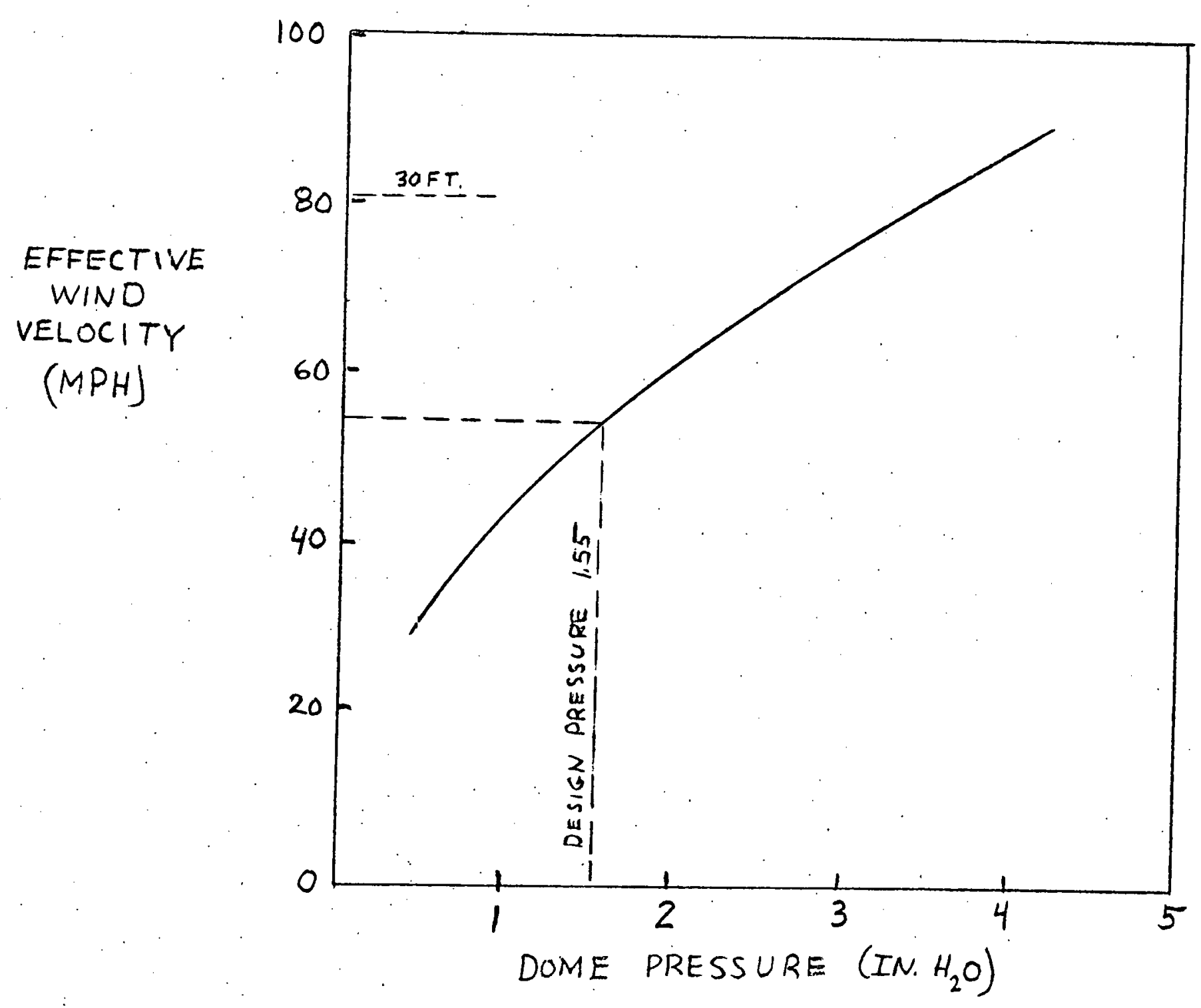

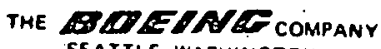


POWER FOR RESEARCH EXPERIMENT BLOWERS

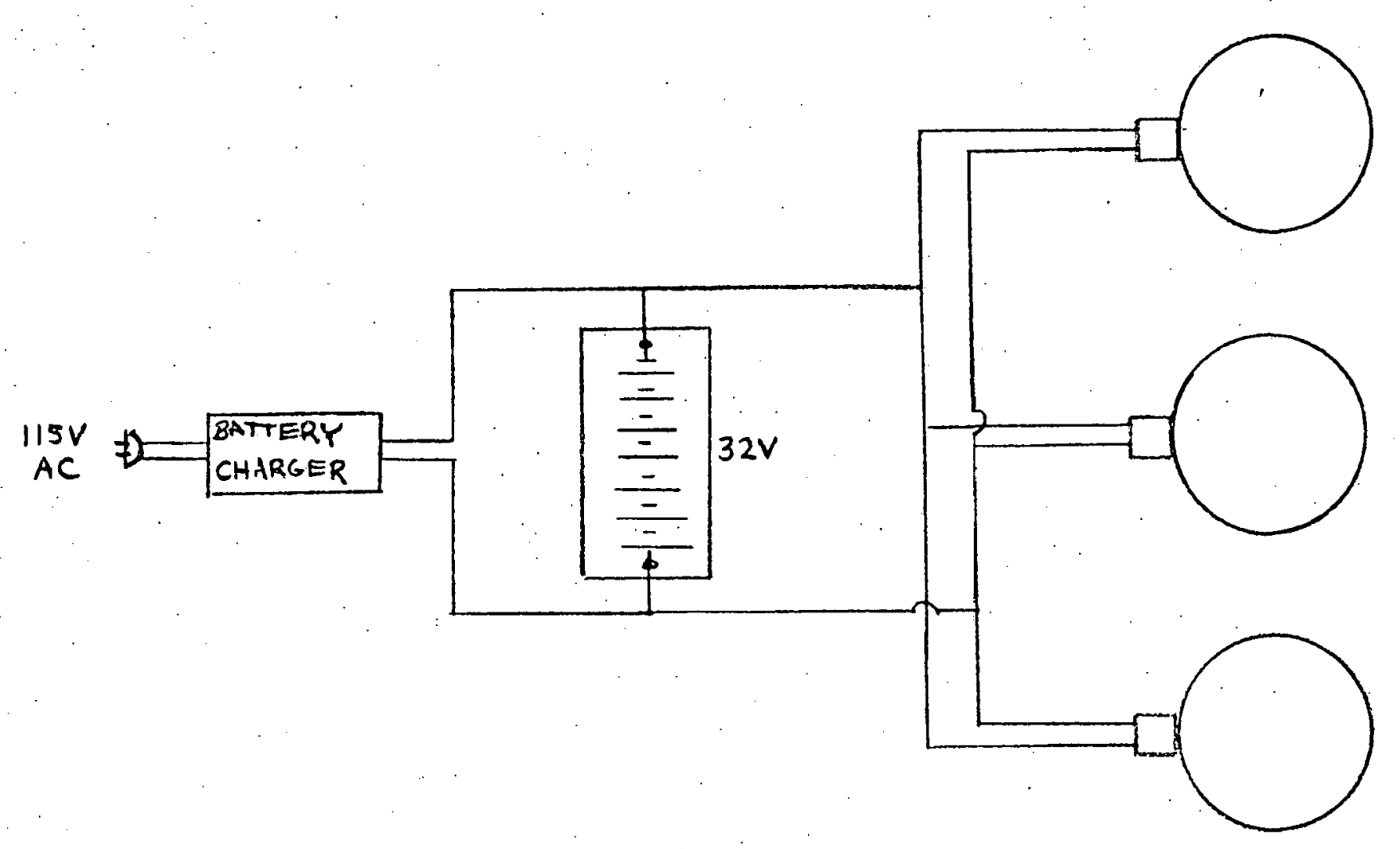




\section{Summary of Dome Material Properties*}

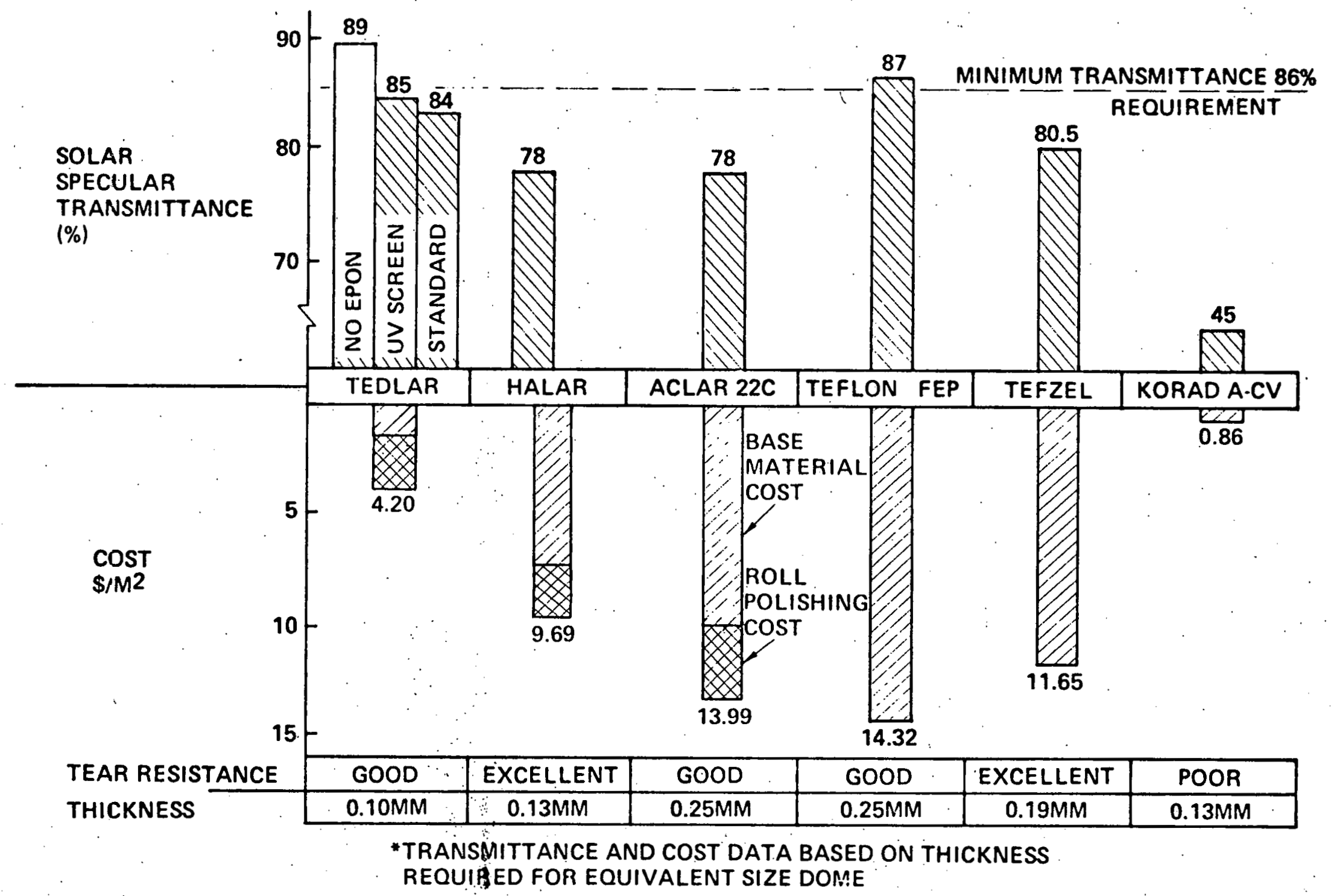




\section{ULTRAVIOLET EFFECTS ON TEDLAR}

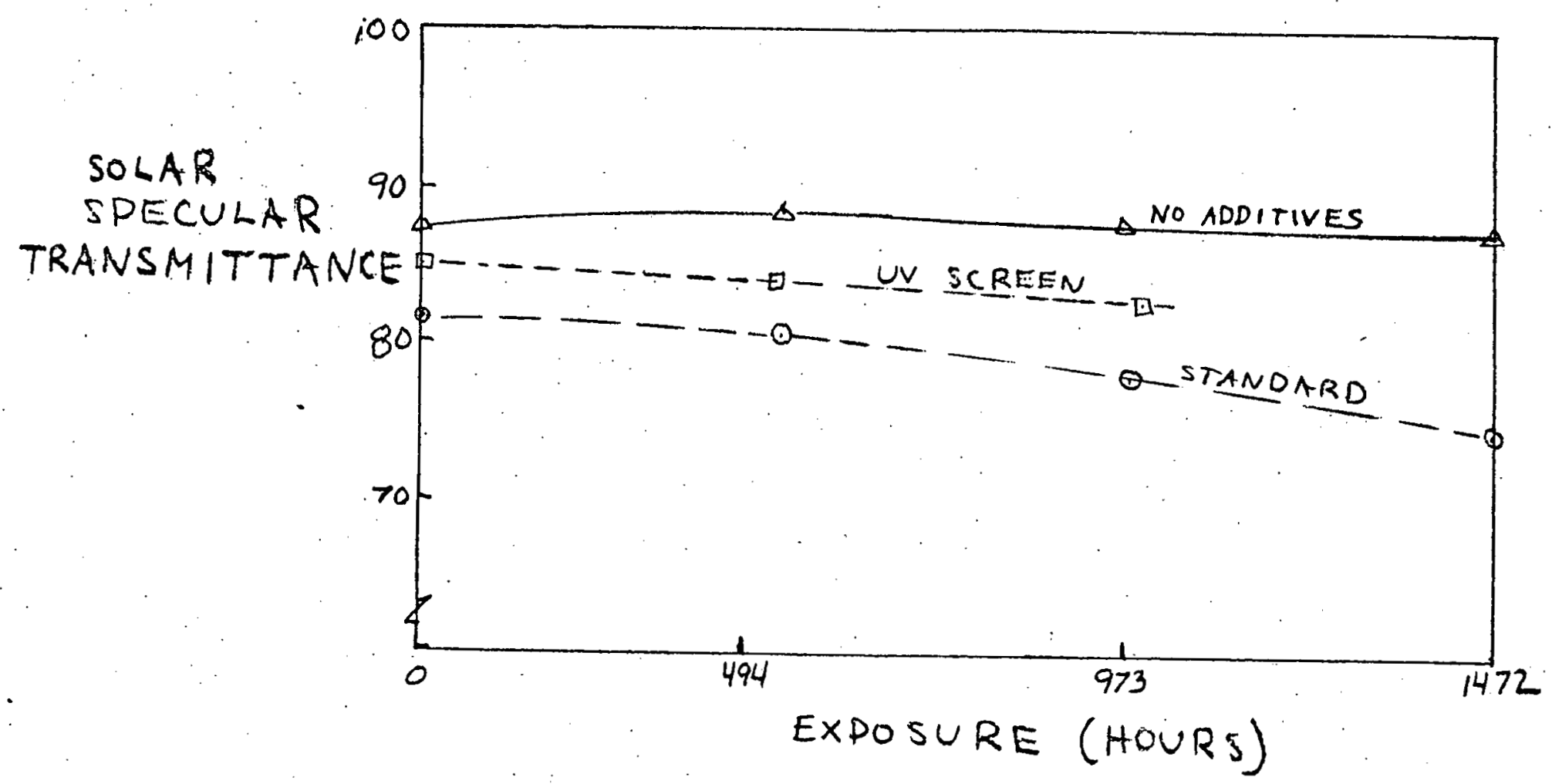




\section{Effect of Incidence Angle on Tedlar Transmittance}

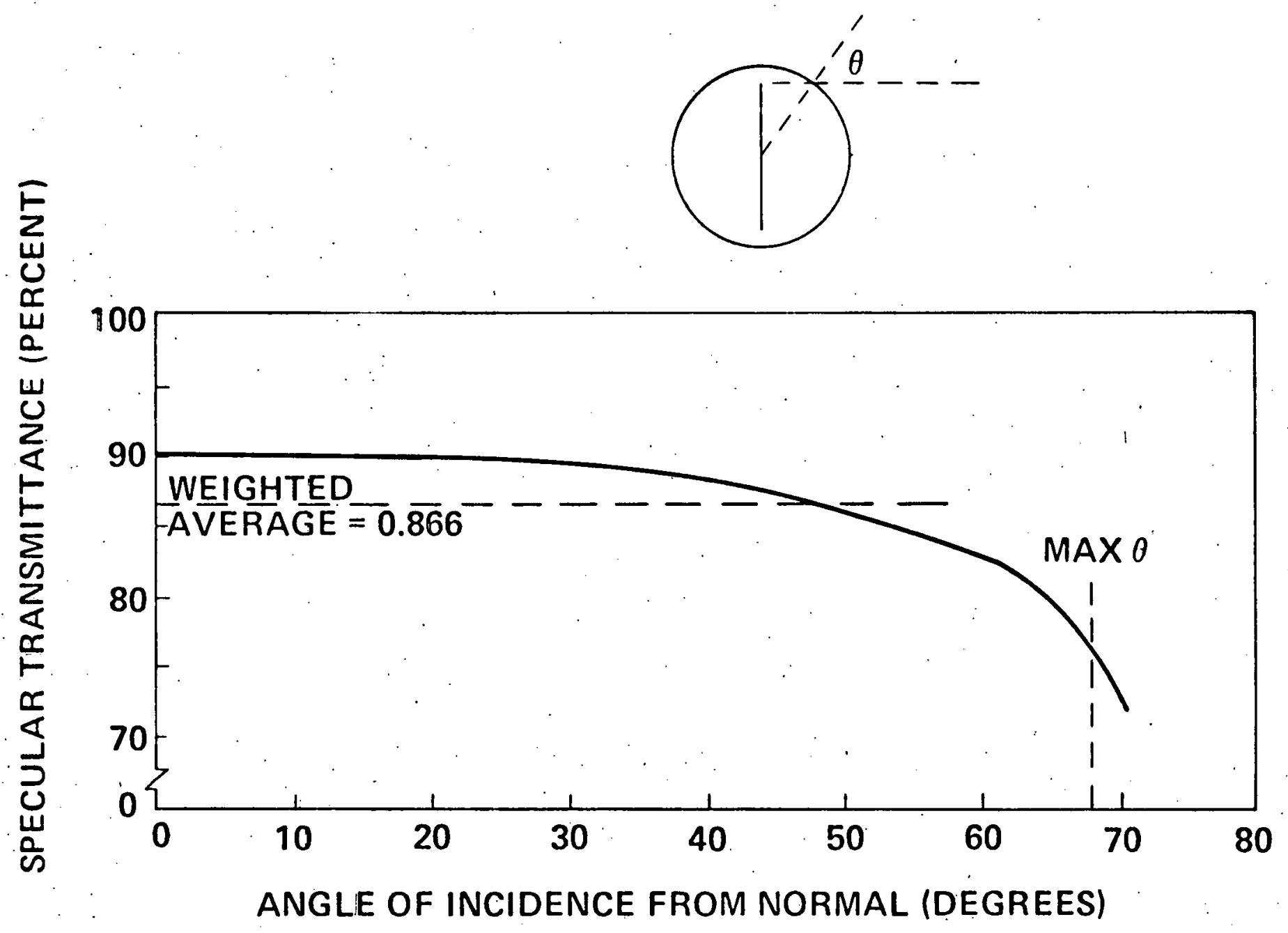


TRANSPARENT ENCLOSURE DESIGN

- Design Environment

- WIND

- Snow/Ice

- Earthquake

- Structural Analyses

- Shape

- SIZE

- Thickness

THE COEOCOCOMPANY 


\section{Collector Subsystem}

\section{Calculation of Effective Wind Velocity for}

Design of Transparent Enclosure

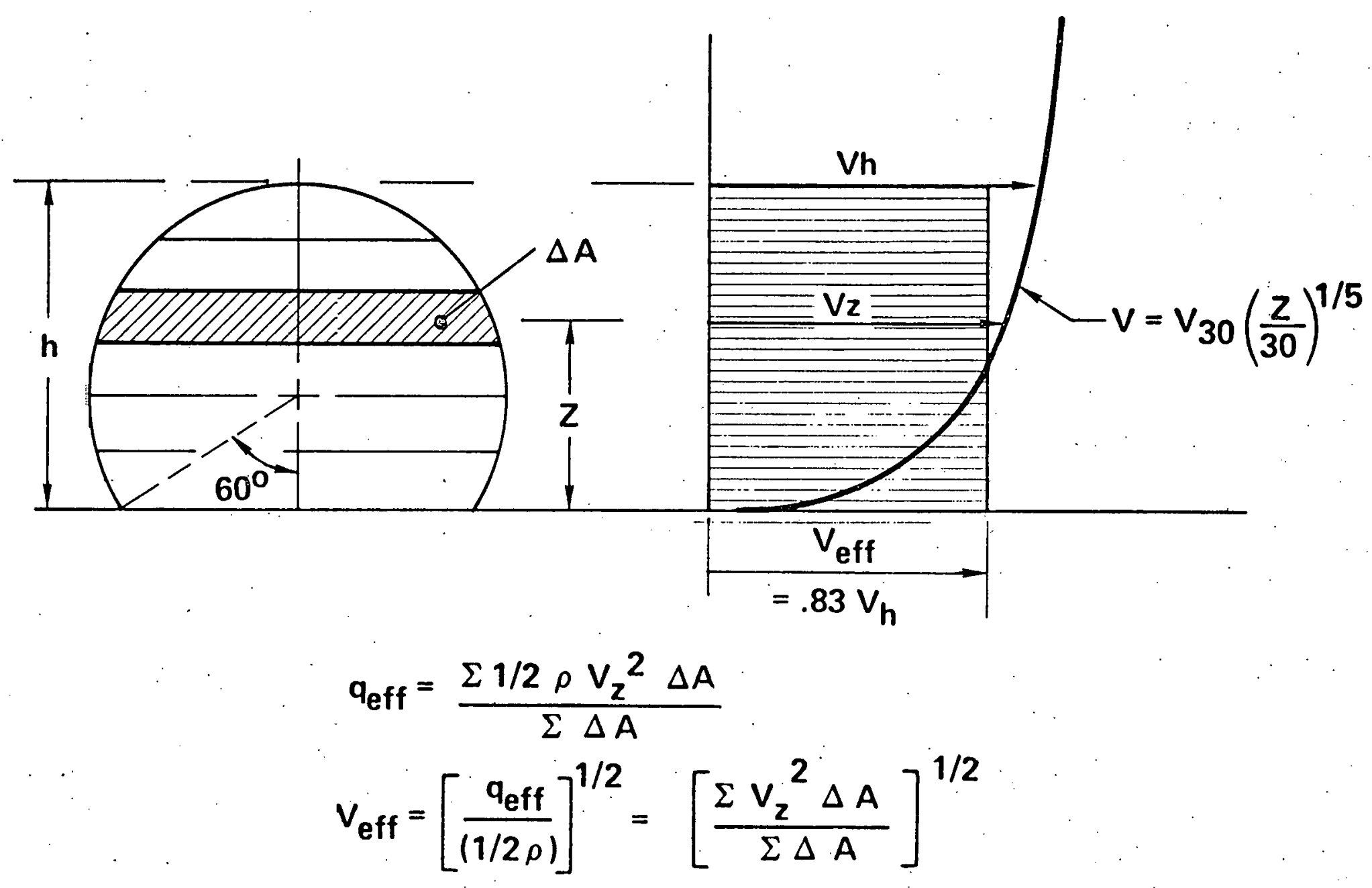




\section{Enclosure Diameter vs Effective Wind Velocity for 4-Mil Tedlar at Various Locations}

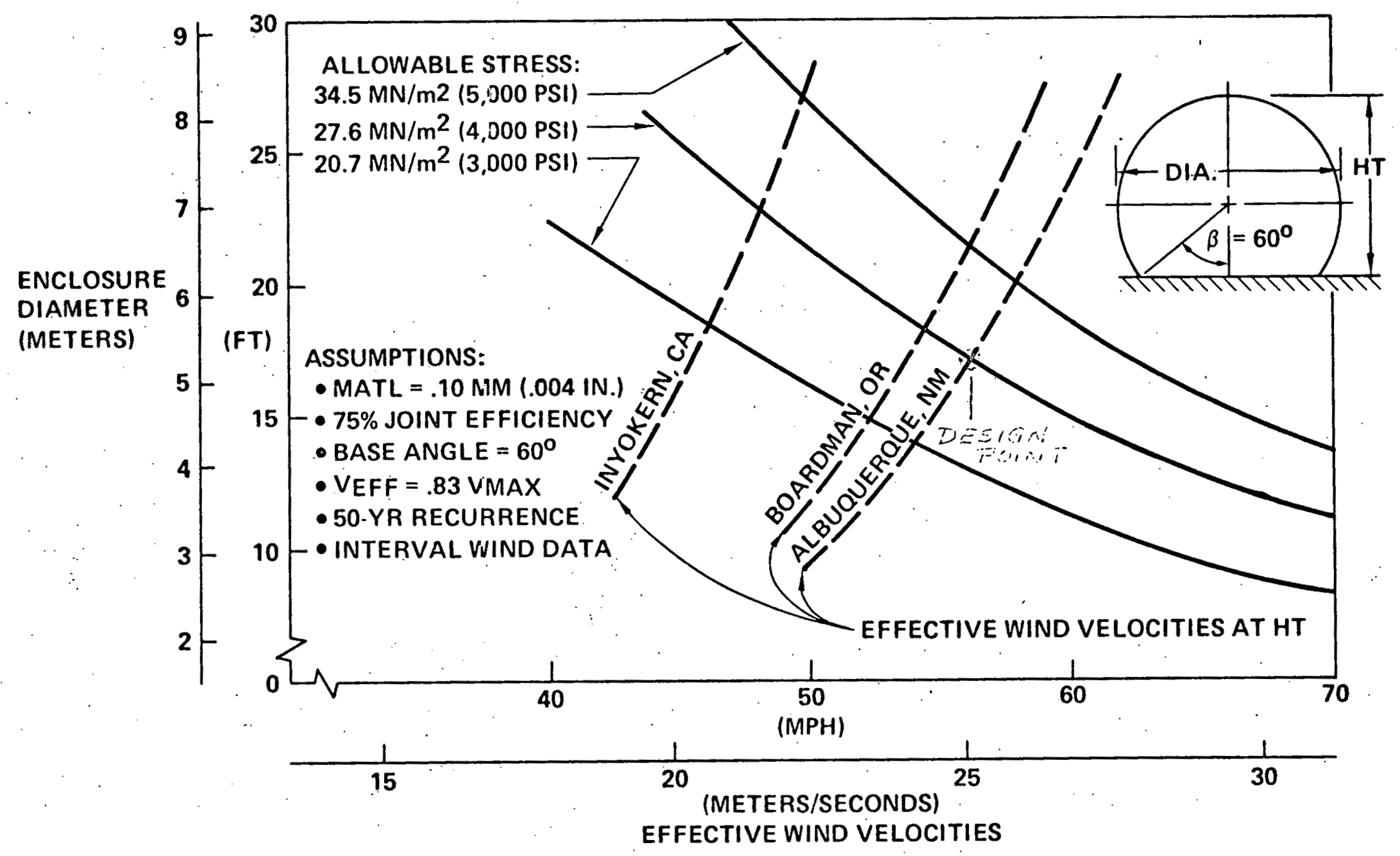


Enclosure material tensile test data

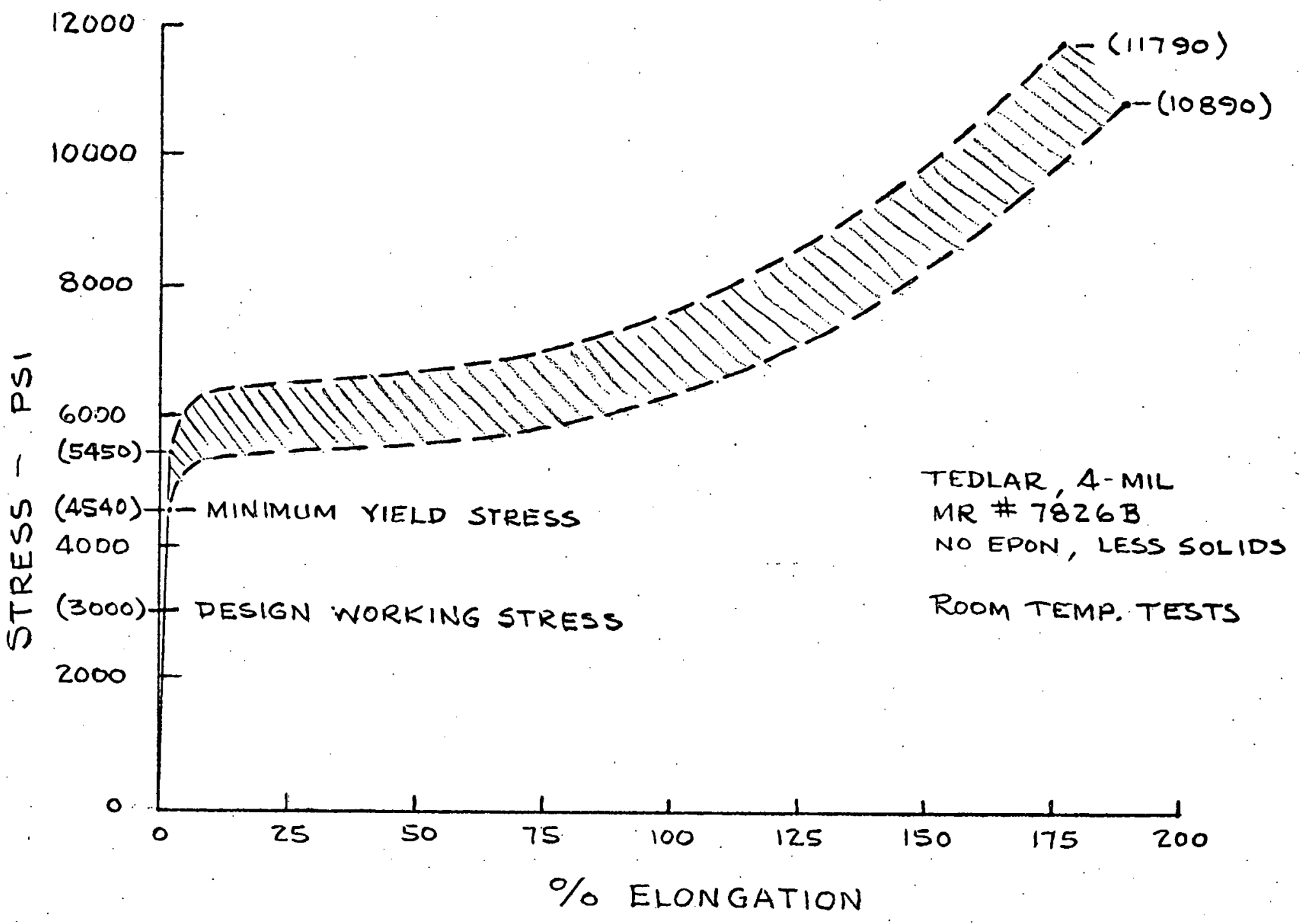

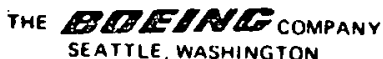


CREEP TEST DATA FOR PEDLAR

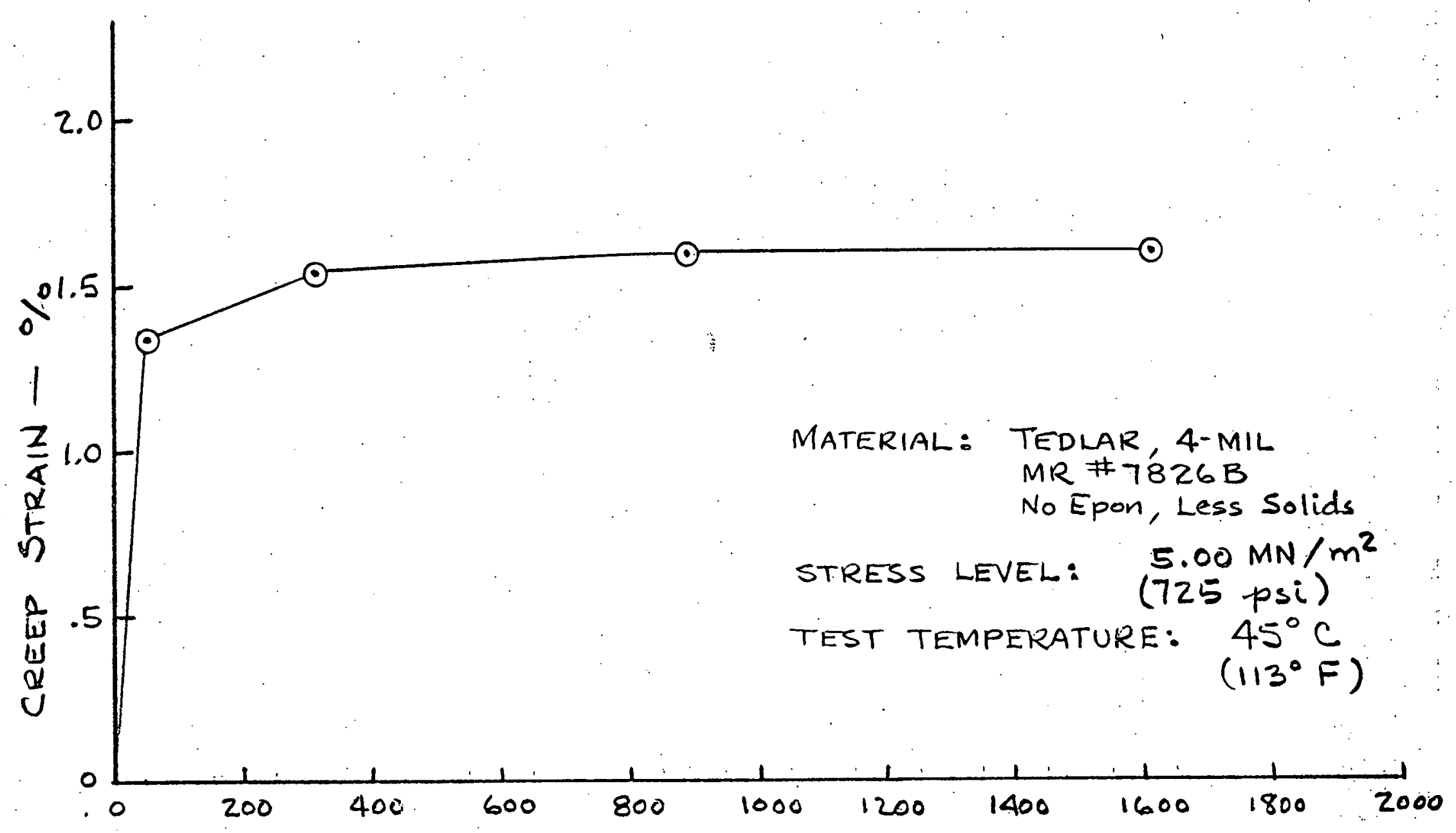

EXPOSURE TIME - HRS

The 20 SEATtLE, WASHINGTON 


\section{ENCLOSURE MATERIAL YIELD STRESS VS TEMPERATURE}

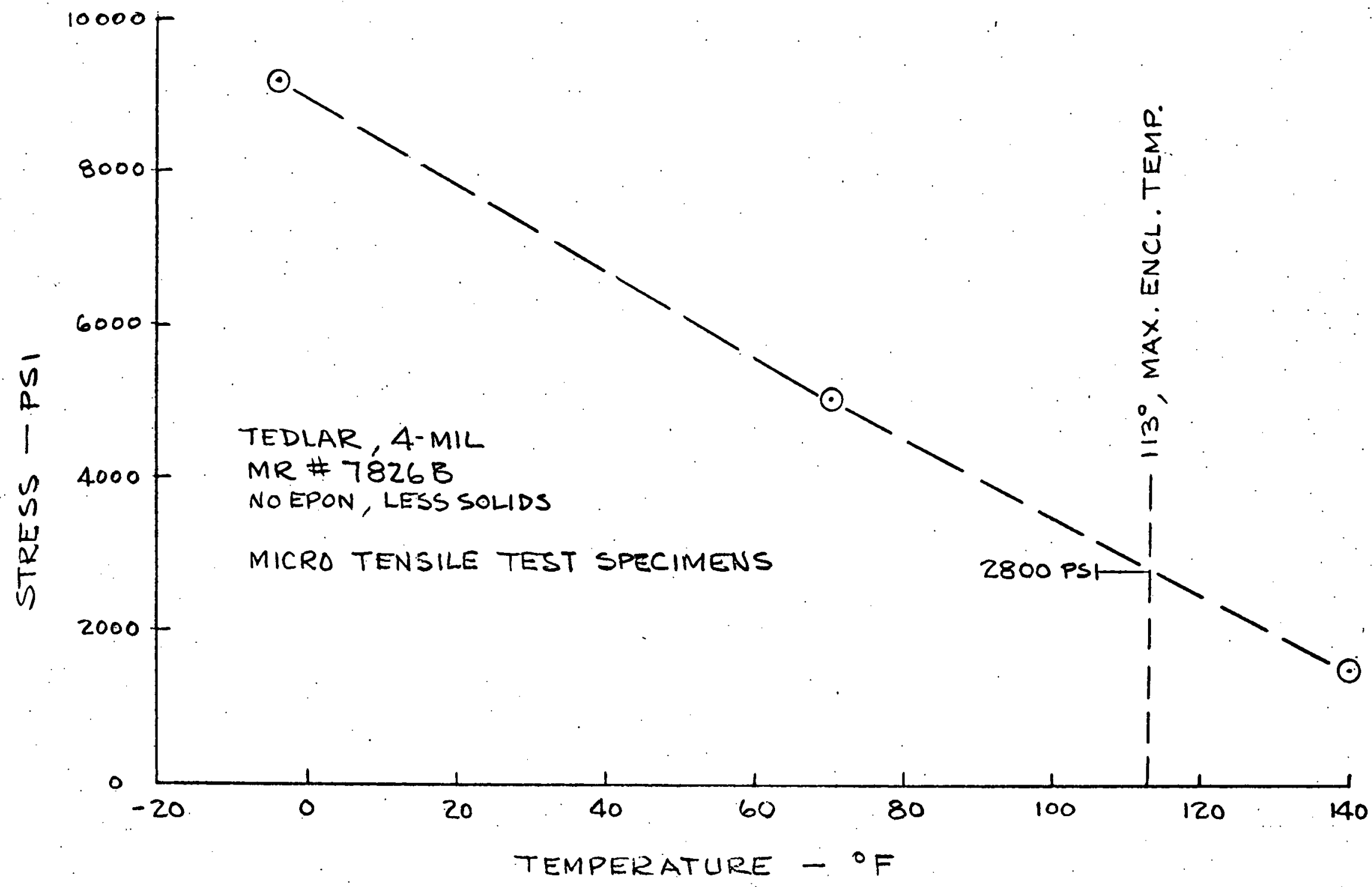


ACCELERATED UV DEGRADATION OF TENSILE PROPERTIES

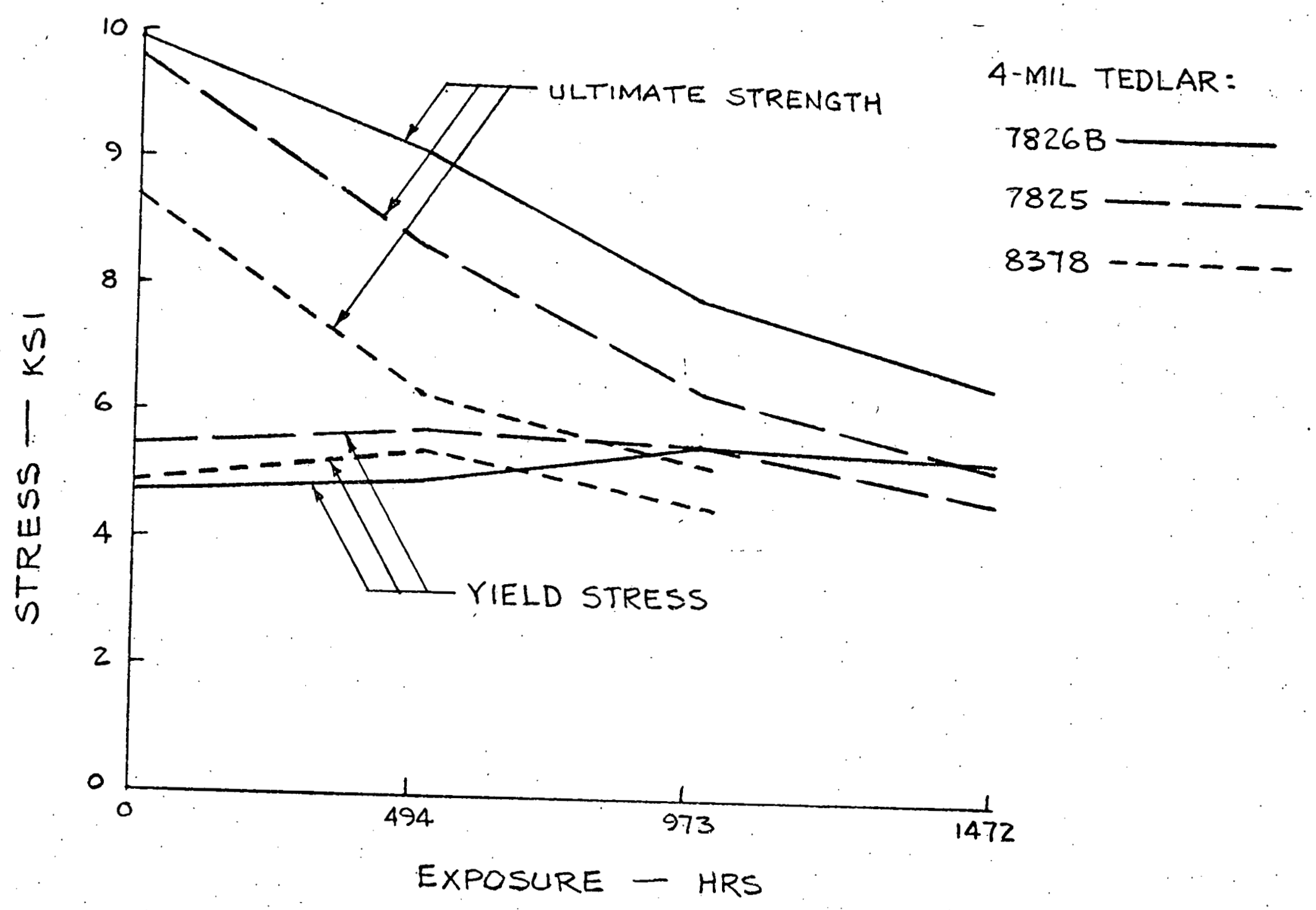


DESIGN REQUIREMENTS - REFLECTIVE ASSEMBLY

SPECULAR SOLAR REFLECTANCE

STRUCTURAL. SUPPORT

LIFETIME

$\operatorname{cosT}$
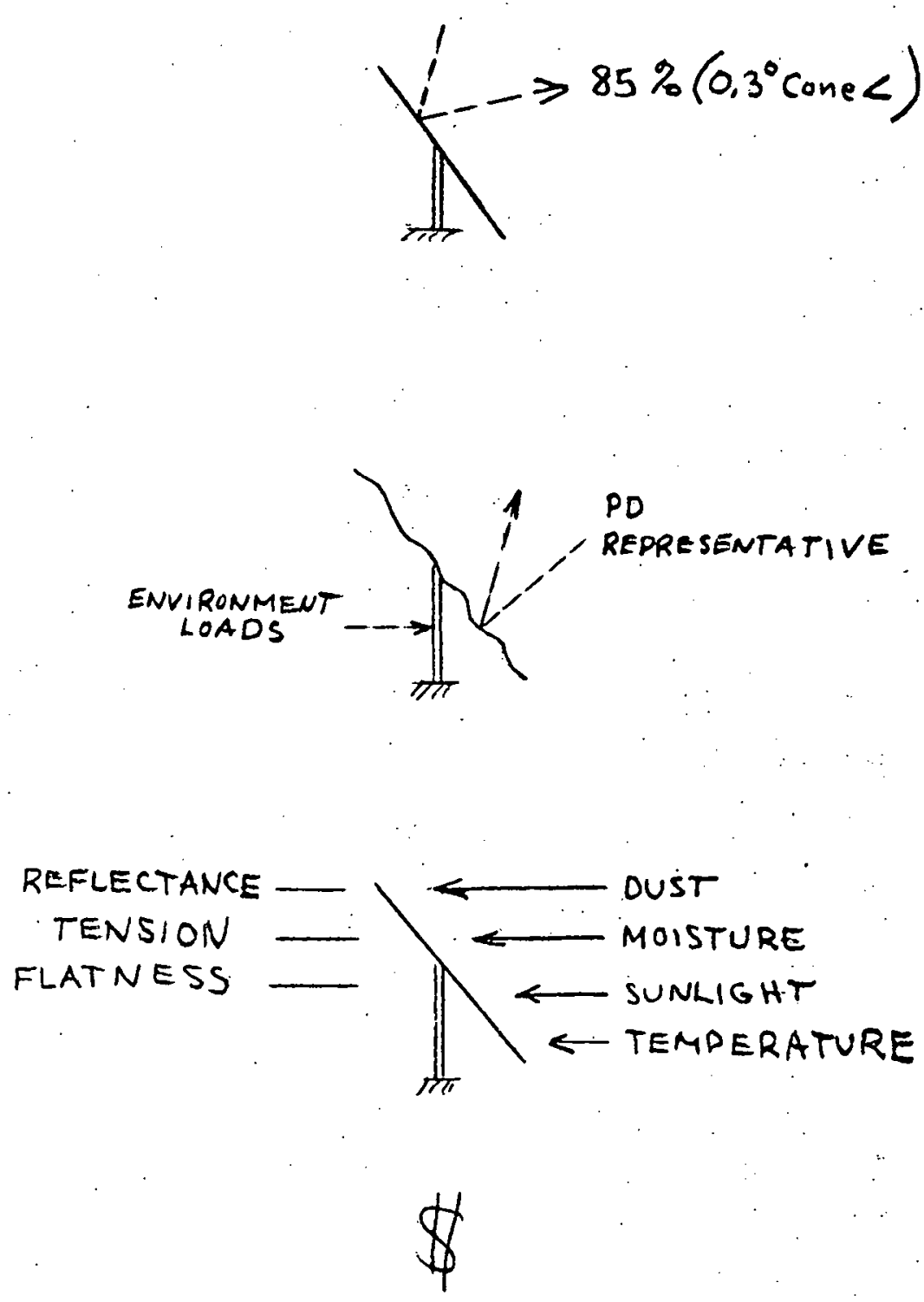


\section{Reflective Assembly Design for Research Experiments}

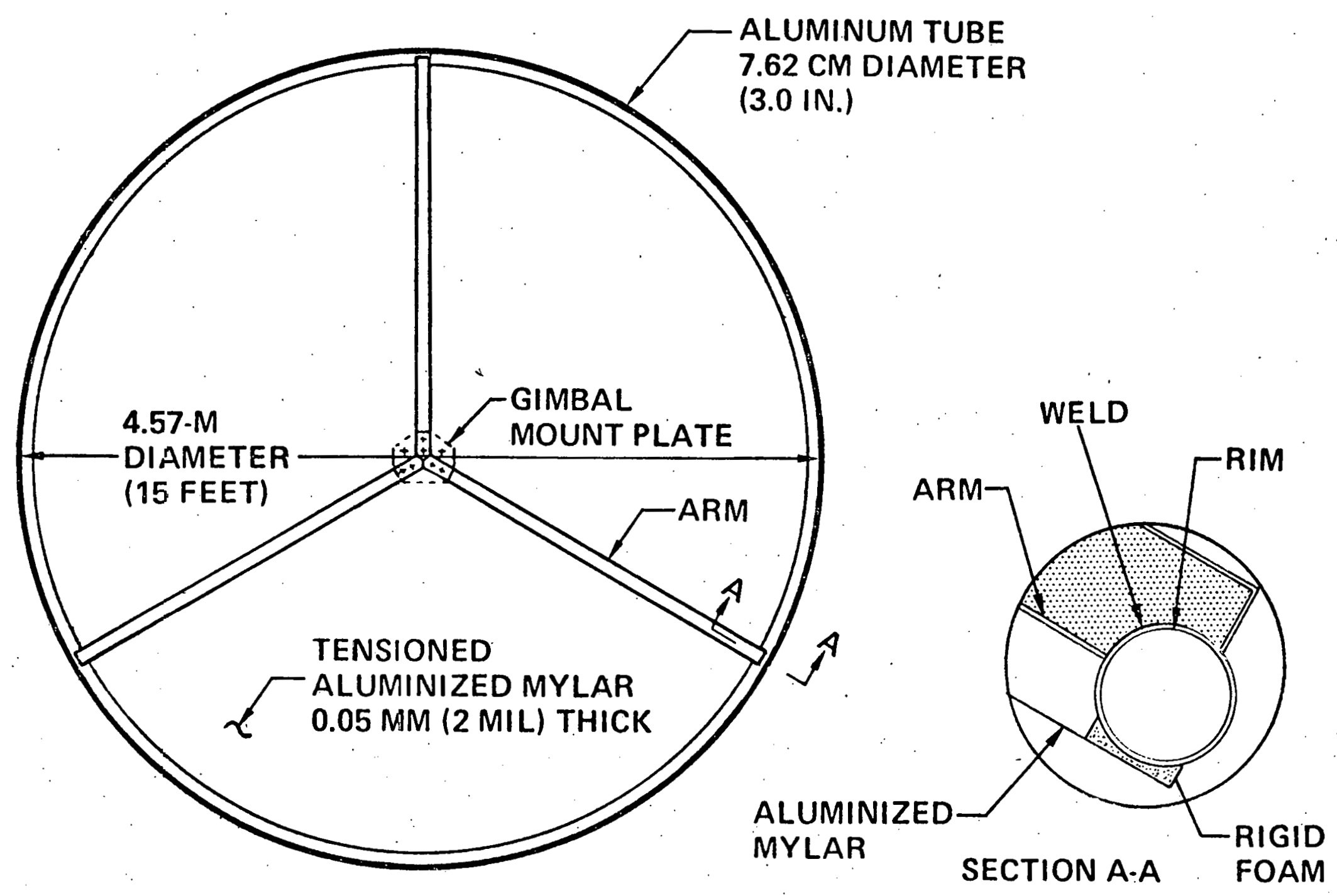

16.24 $M^{2}$ AREA 


\section{Reflector Material Properties}

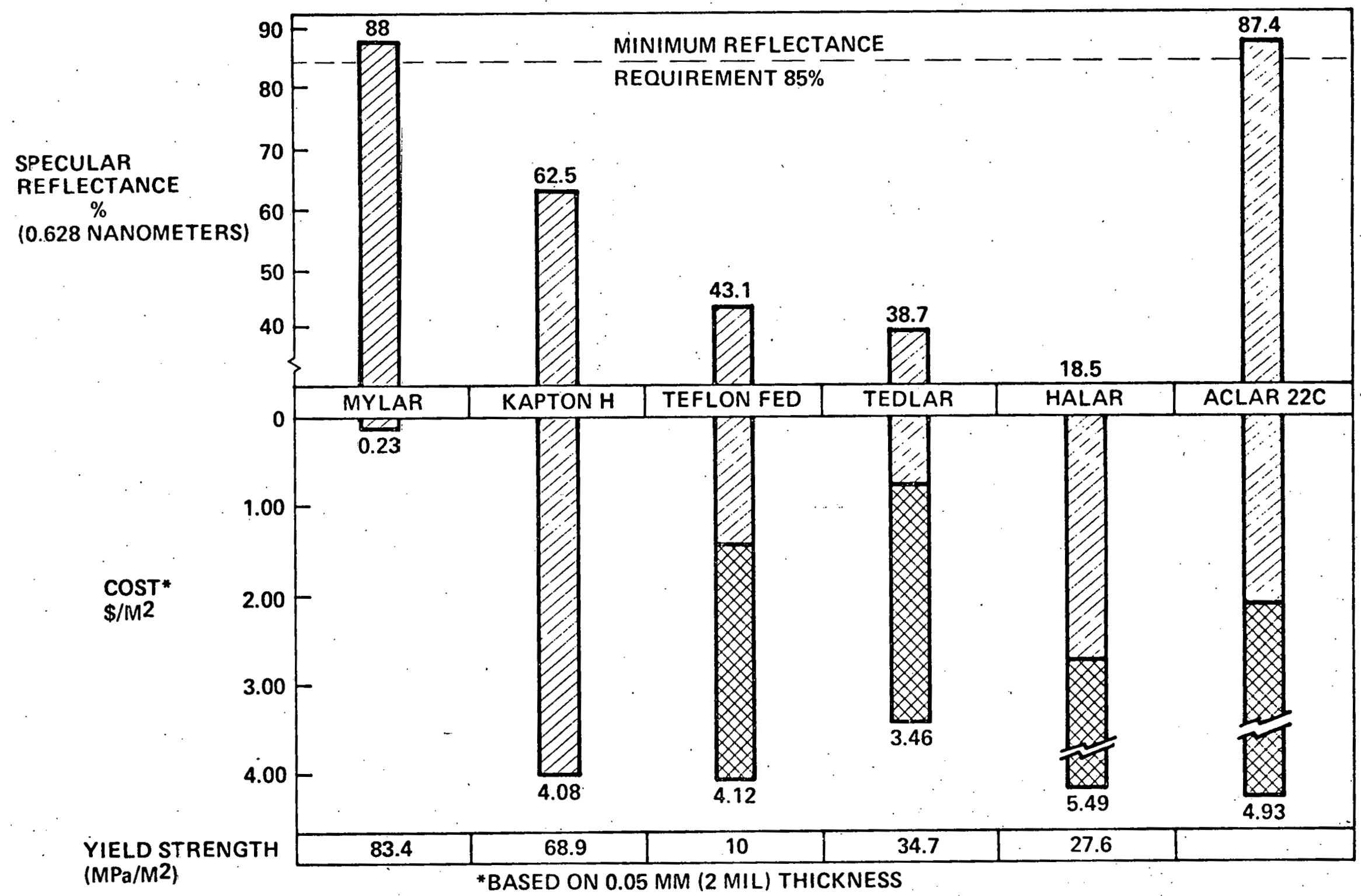


STRUCTURAL DESIGN OF REFLECTIVE ASSEMBLY

gravity deflection of membrane

variation in stress due to charges in environment

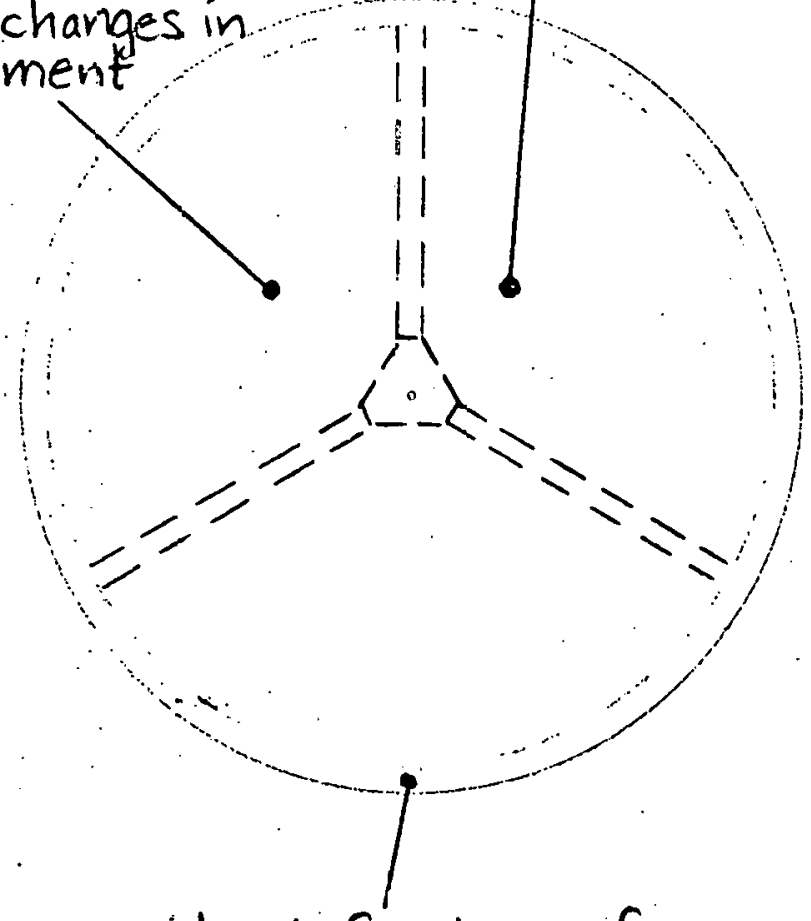

gravity deflection of support ring relative deflection for wind and earth quake loads

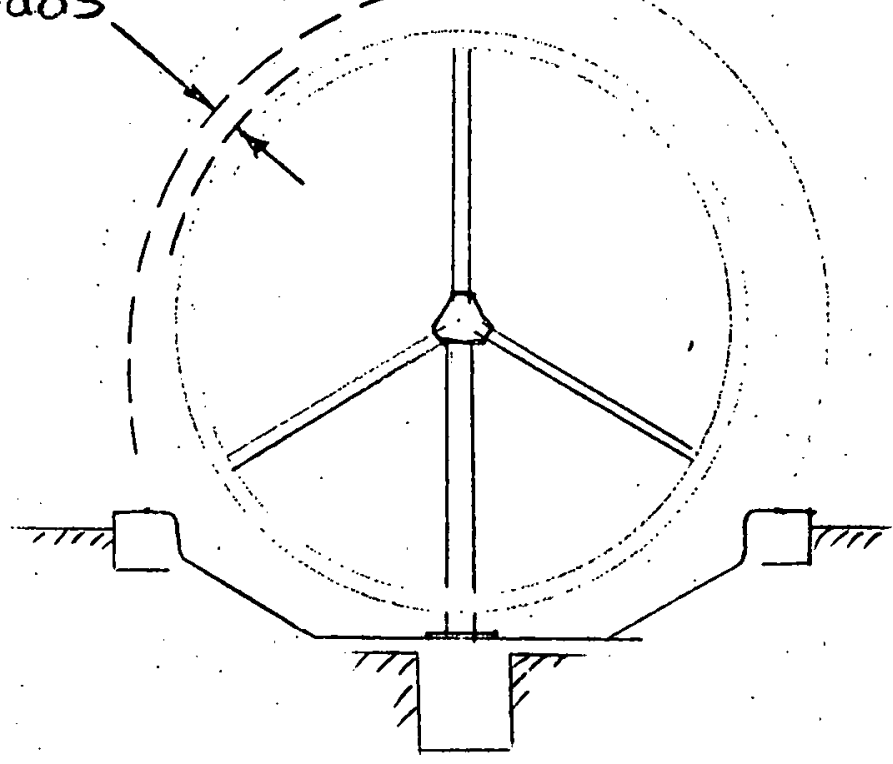

THE GETTER WASHINGTOMPANY 
ENVIRONMENTAL EFFECTS ON MEMBRANE TENSION

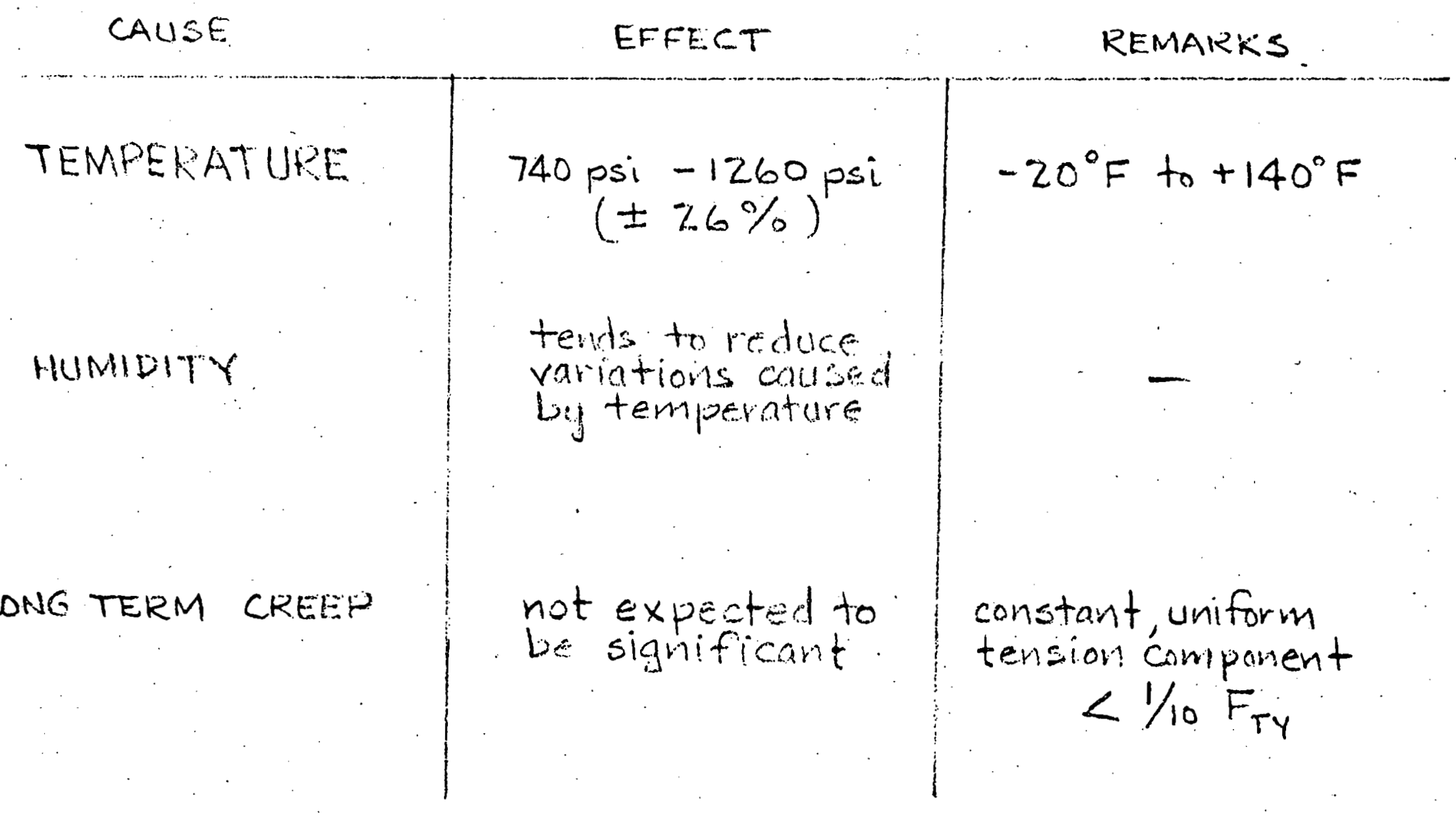

LONG TERM CREEP

not expected to

constant, uniform tension component $<1 / 10 F_{\text {TY }}$

THE 200 SEATtLE, WASHINGTON 


\section{CREEP TEST DATA FOR MYLAR}

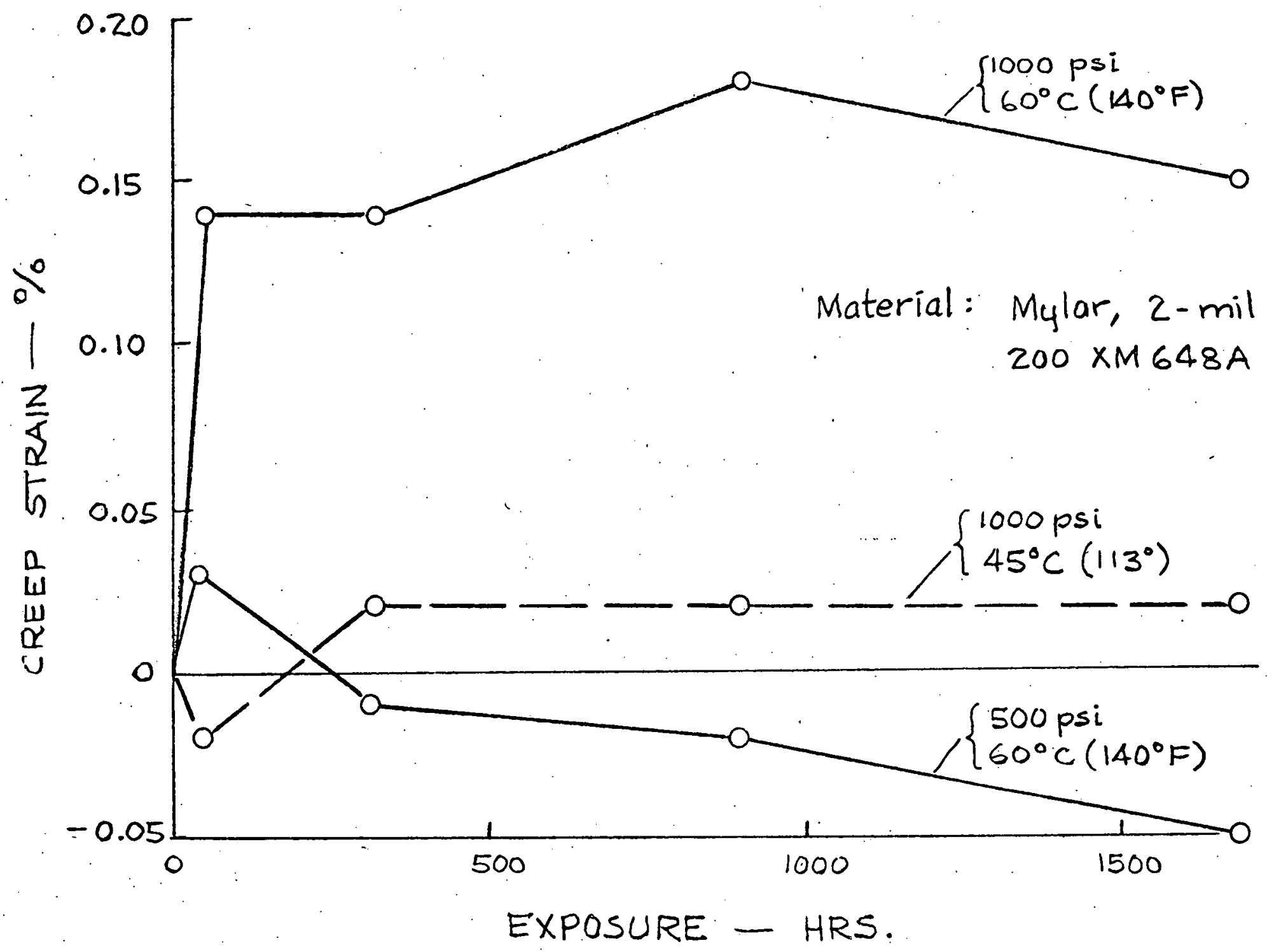




\section{Membrane Deflection Due to Gravity- Circular Reflector}

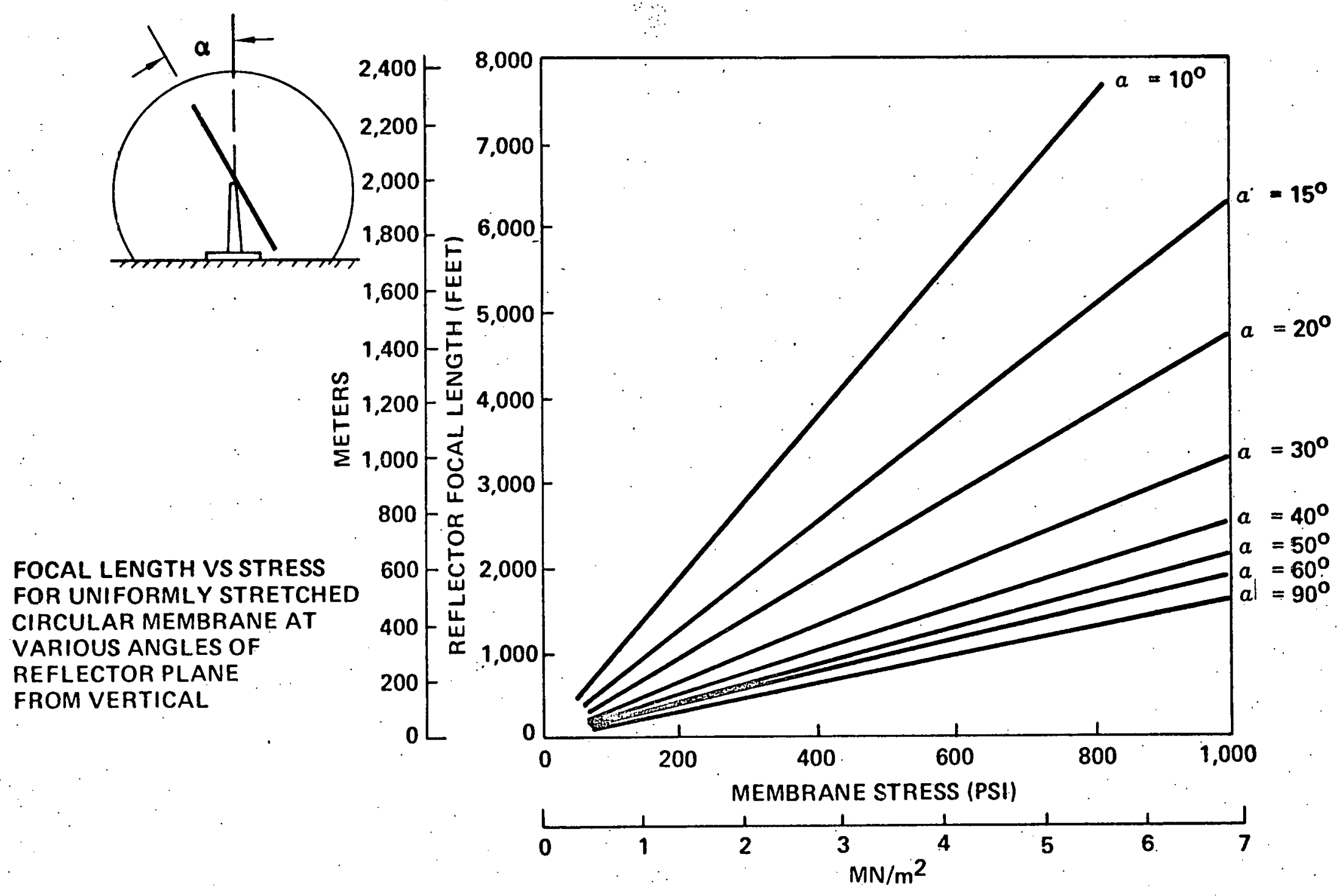


Reflector Response to Single Step Showing Alternative Phasings for Beginning Subsequent Step

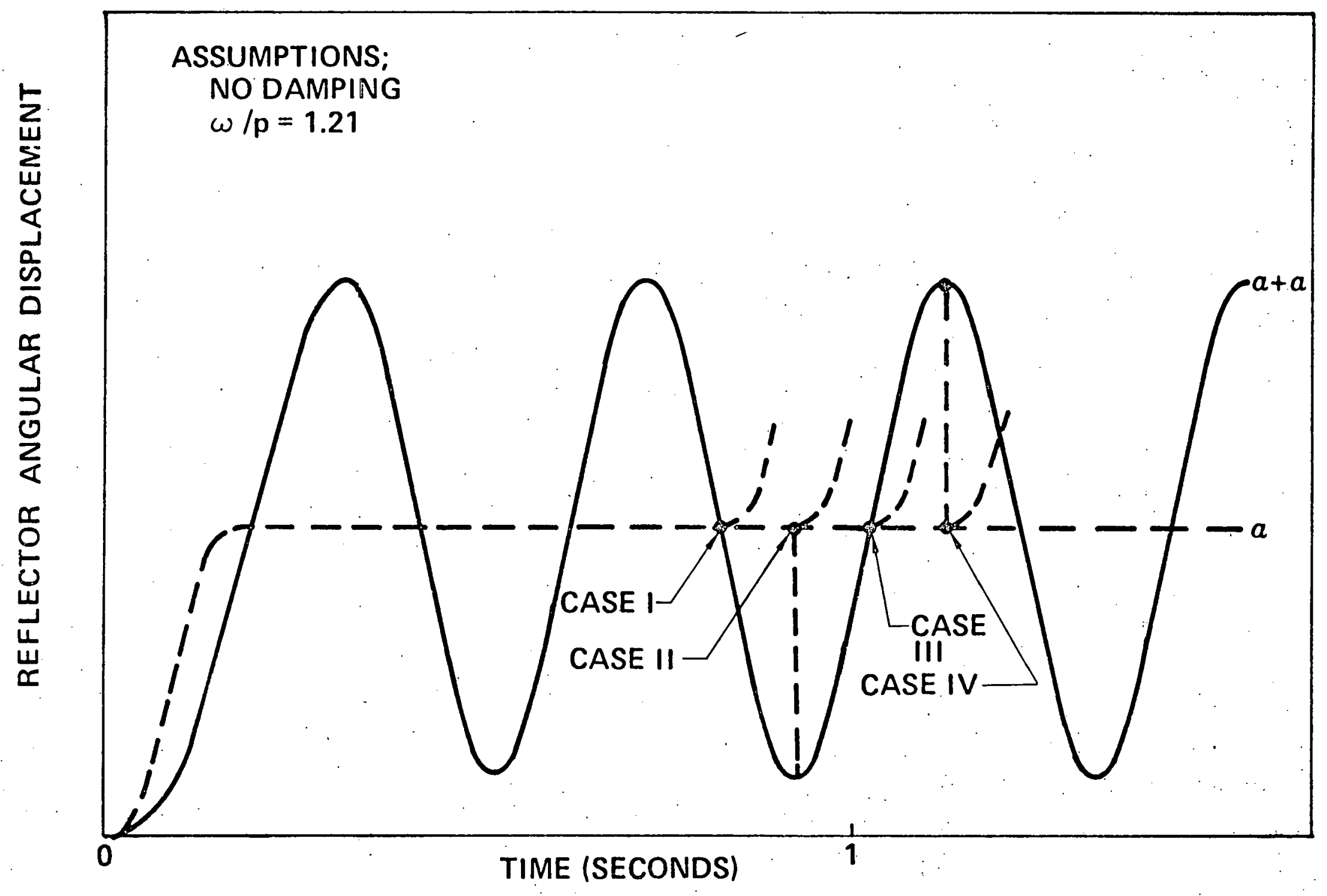




\section{Reflector Response versus Number of Consecutive}

Steps for Different Phasings

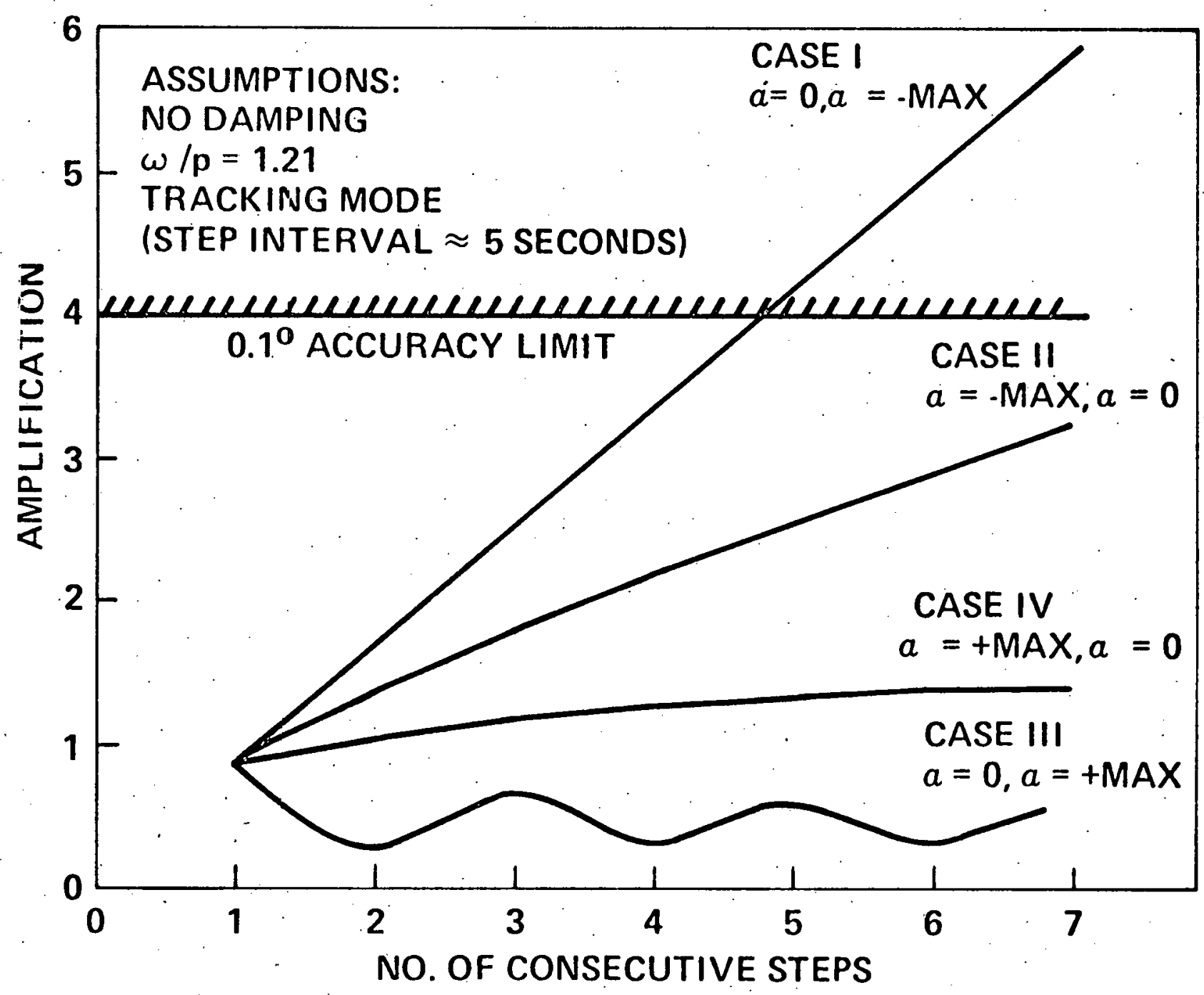


LRIVE \& CONTROL ASSEMBLY RENUIRENENTS

- Provide MODES of OPERATION

- TRACK

- STANDBY

- ALIGMENT

- SHUTDOWN

- MANUAL

- PROVIDE $\pm 0.05^{\circ}$ ORIENTATION ACCURACY

- FROVIDE $0.125^{\circ} / \mathrm{SEC}$ SLE!! RATE CAPABILITY 
RESEARCH EXPERIMENTS

DRIVE \& CONTROL SYSTEM SCHEMATIC

i

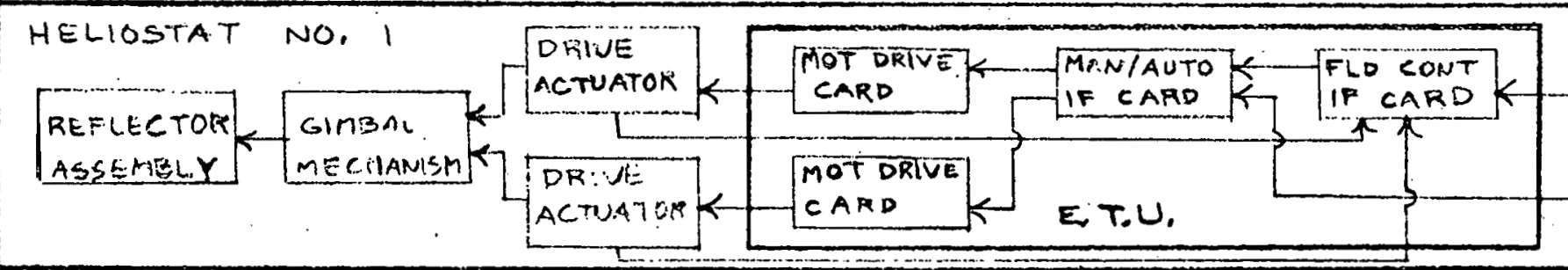

MAN CONT

UNIT
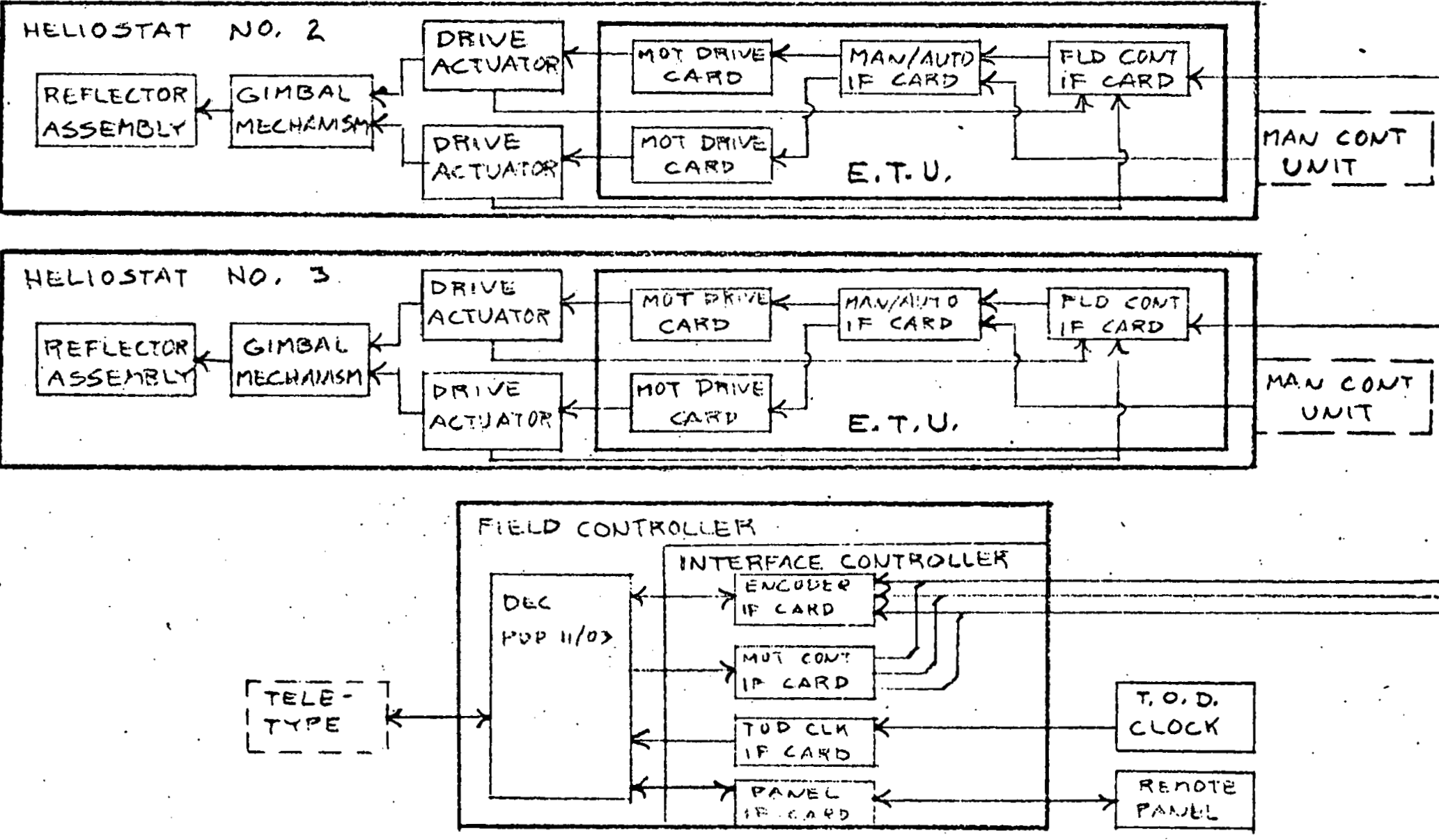

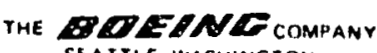

SEATILE. Wasmington 


\section{REFLECTOR STEP TRACKING}

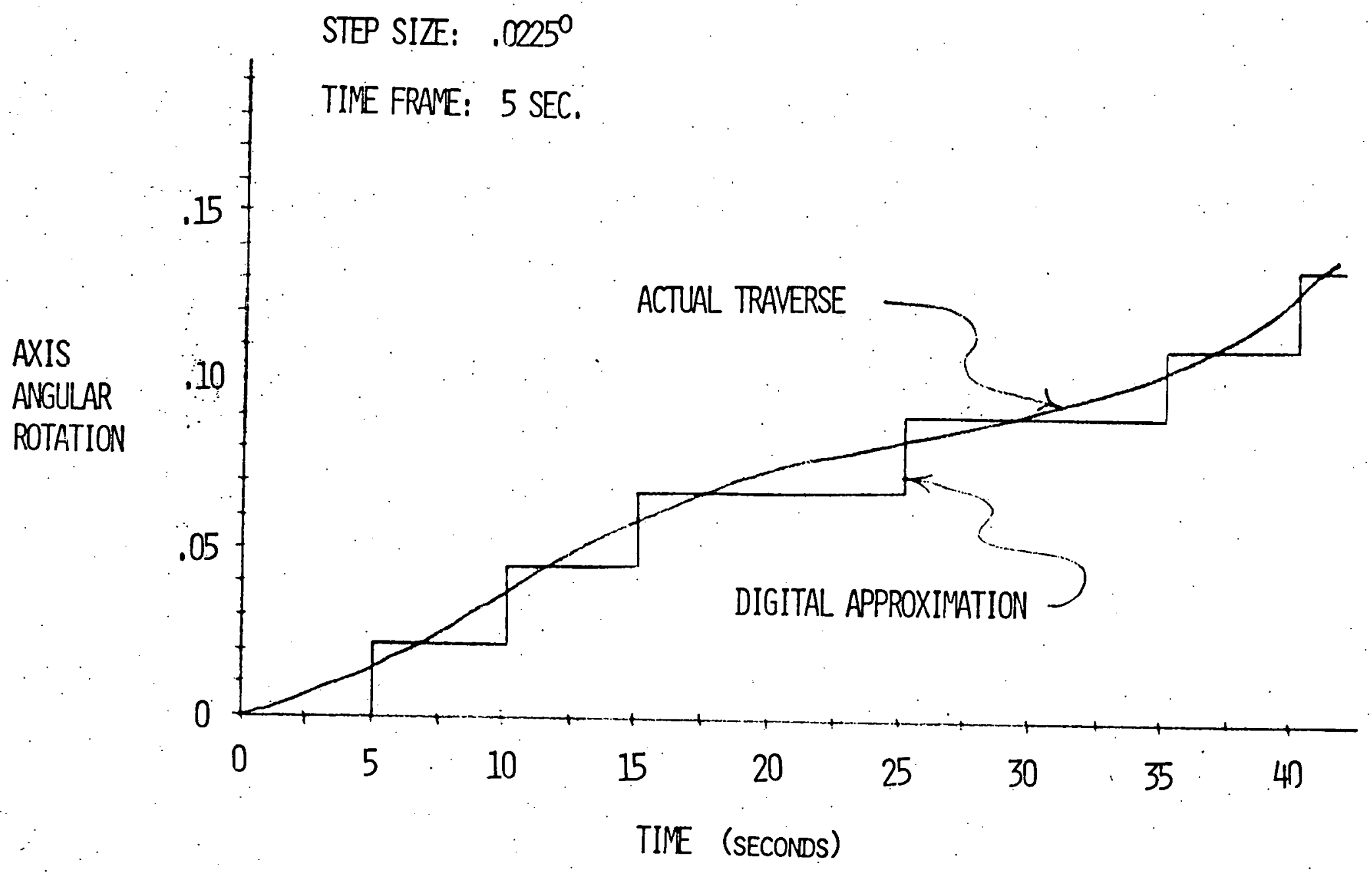

the Materevecompant 
CONTROLS ASSEMBLY

Drawing Tree

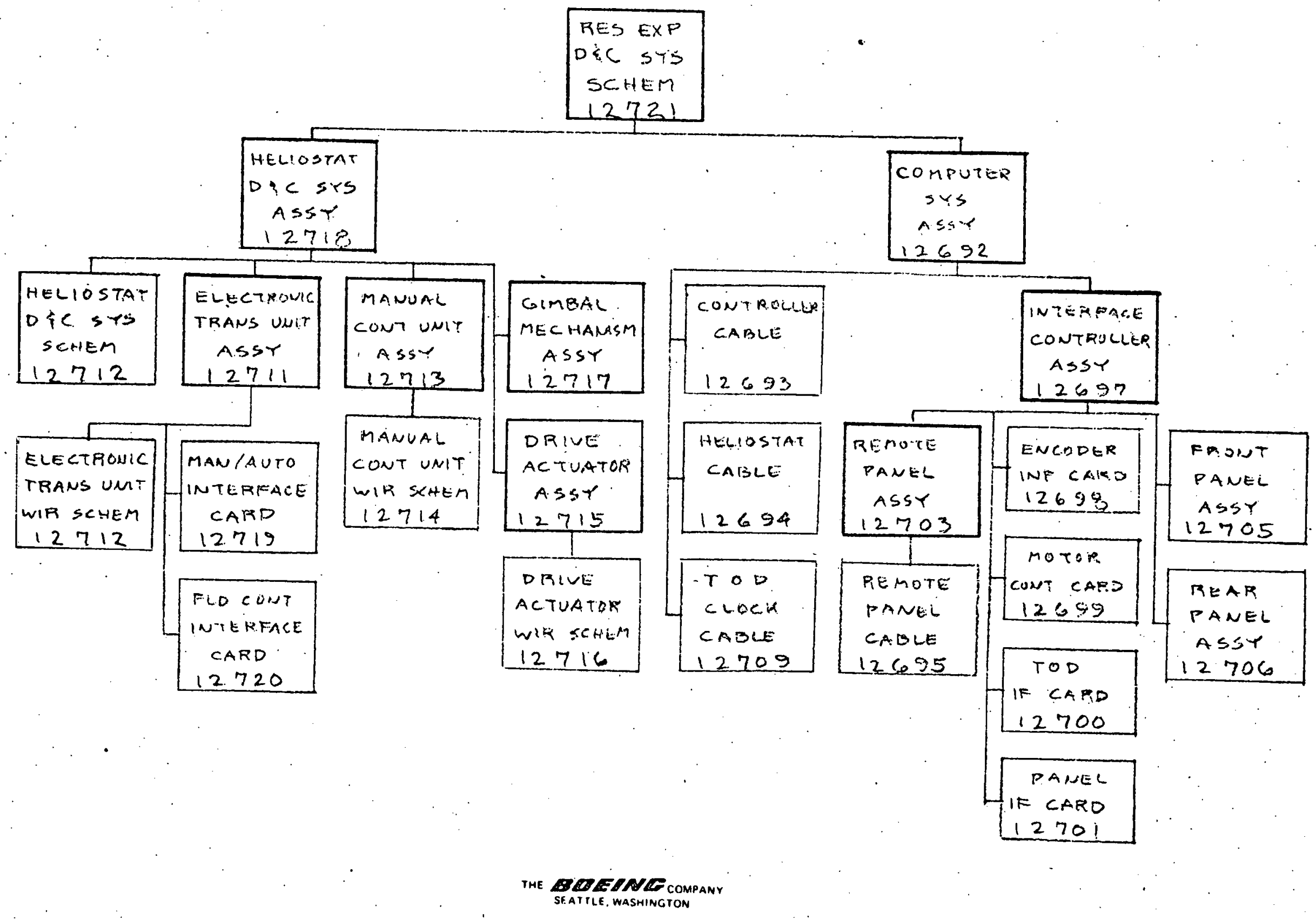




\section{Drive and Control Assembly}

1. Drive actuator (2/heliostat)
A. Stepper motor
B. Harmonic drive actuator
C. Drive bearings
D. Optical encoder

2. Drive structural support (1/heliostat)

A. 2-axis gimbal mechanism

B. rounterweight

3. Heliostat control transmission (1/heliostat)

A. Electronic control box

1) Motor driye cards

2) Manual/automatic interface card

3) Computer decoder card

4) Power supply modules

B. Manual remote control box

C. Limit switches

4. Central controls interface

A. Field microprocessor controller

1) Electronic chips: CPU, PROM, RAM, etc.

2) Power supply modules

3) Ephemeris data

4) Processor equations and programming

B. Cabling and wiring

5. Alignment 


\section{Gimbal Configuration}

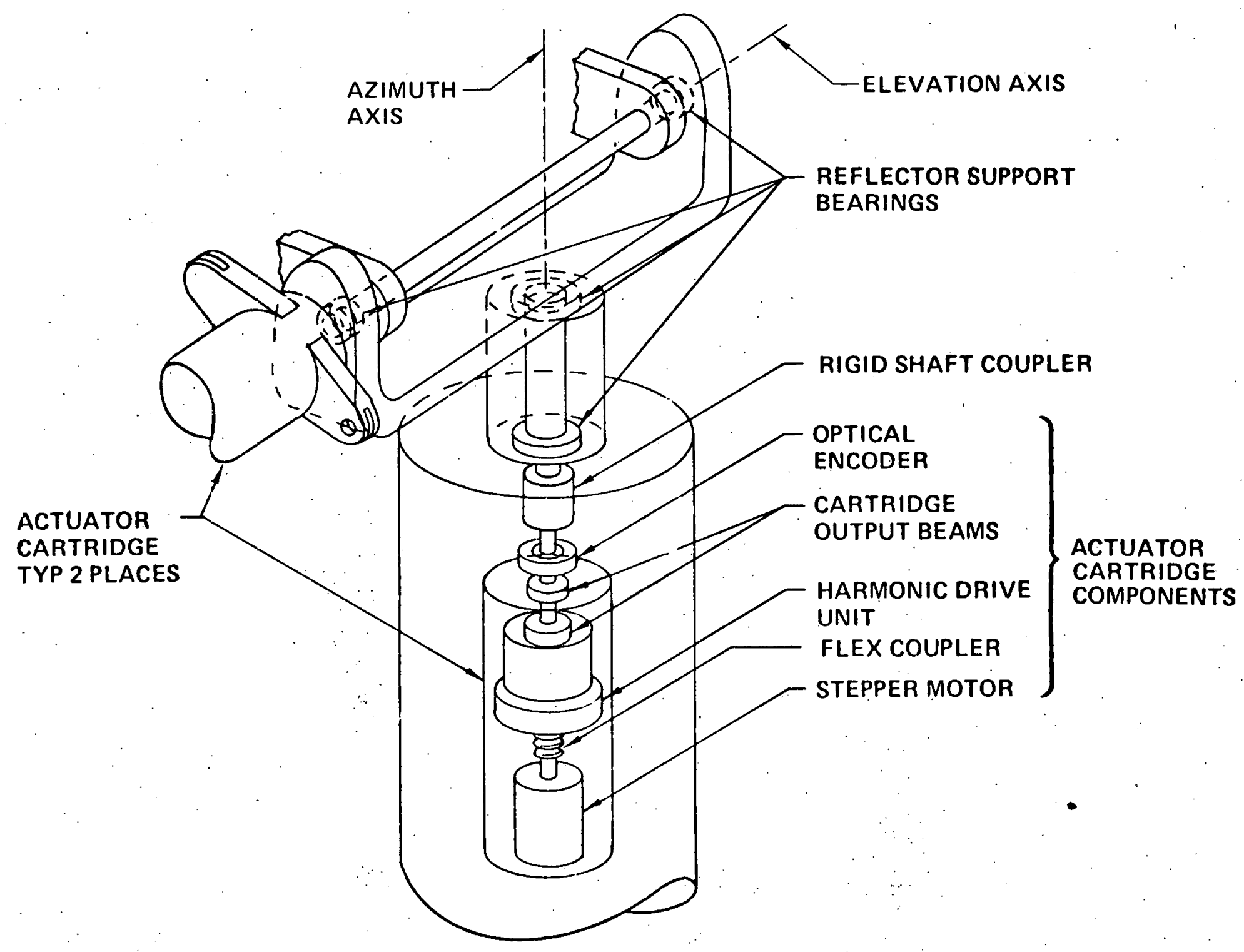




\title{
DRIVE ACTUATOR EEATURES
}

- STEPPER MDTOR

\author{
$1.3^{\circ} /$ STEP \\ 4.) OZ-IN OUTPUT TOROUE \\ DETENT TORRUE \\ BI-DIRECTIOHAL
}

- hARMONIC DRIVE ACTIATOR

8O/1 DRIVE ACTUATOR

209. IIHLR OUTPIT TORQUE

- optical encoDer

1000 CYCLES/REVOLUTIONN RESOLUTION

$T$ T L COPPATIBILITY

SELF-CONTAINED FLECTRONICS

- .025\%/SteP ON OITPIJT SHAFT

- INTERCHANGEABLE CARTRIDGE ASSEMBLY 


\section{Heliostat Drive/Control Block Diagram}

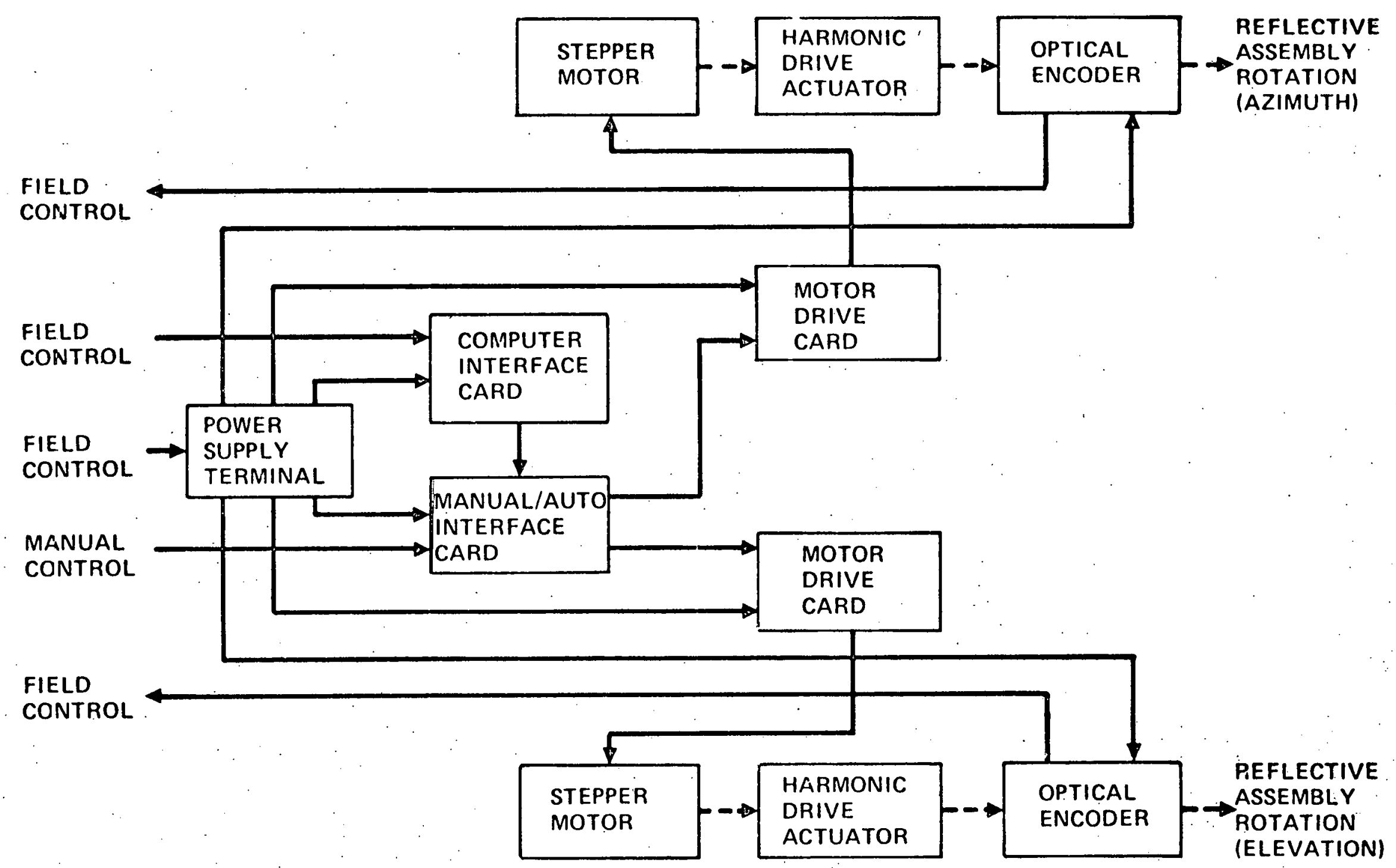




\section{E! ECTRONIC TRANSMISSION UNII FEATURES}

- PORTABLE, LIGHT:EIGHT ENCLOSURE

- EASILY REPLACEABLE CIRCUIT CARDS

- CONNECTORS FOR HELIOSTAT \& POMER CABLES

- LONERS FOR PONER SUPPLY VENTILATION

- terMinal boards to facilitate viring

- OVERVOLTAGE PROTECTION ON POMER SUPPLIES 


\section{MANUAL CONTROL UNIT FEATURES}

- PORTABLE, RUGGED, LIGHTYEIGHT S:IITCHBOX

- 10 FT. CABLE WITH CONNECTOR

- 3 POSITION MODE SEECTOR SWITCH

AUTO, MAN, OFF.

- SPRING RETURN TO CENTER CONTROL SMITCHES

UP/DOWN

LEFT/RIGHT 


\section{FIELN CONTROLLER FEATIRES}

- DEC FDP 11/03 DIGITAL MINI-COMPIJTER

- intepface controller

$$
\begin{aligned}
& \text { MODE SELECT SUITCH } \\
& \text { MONITOR IIDICATORS } \\
& \text { MA://AACHIIE INTERFACE }
\end{aligned}
$$

- TIME OF DAY CLOCK

- REPDTE UNIT (ALIGNMENT)

- CABLING

- SOF WARE PROGRAM 


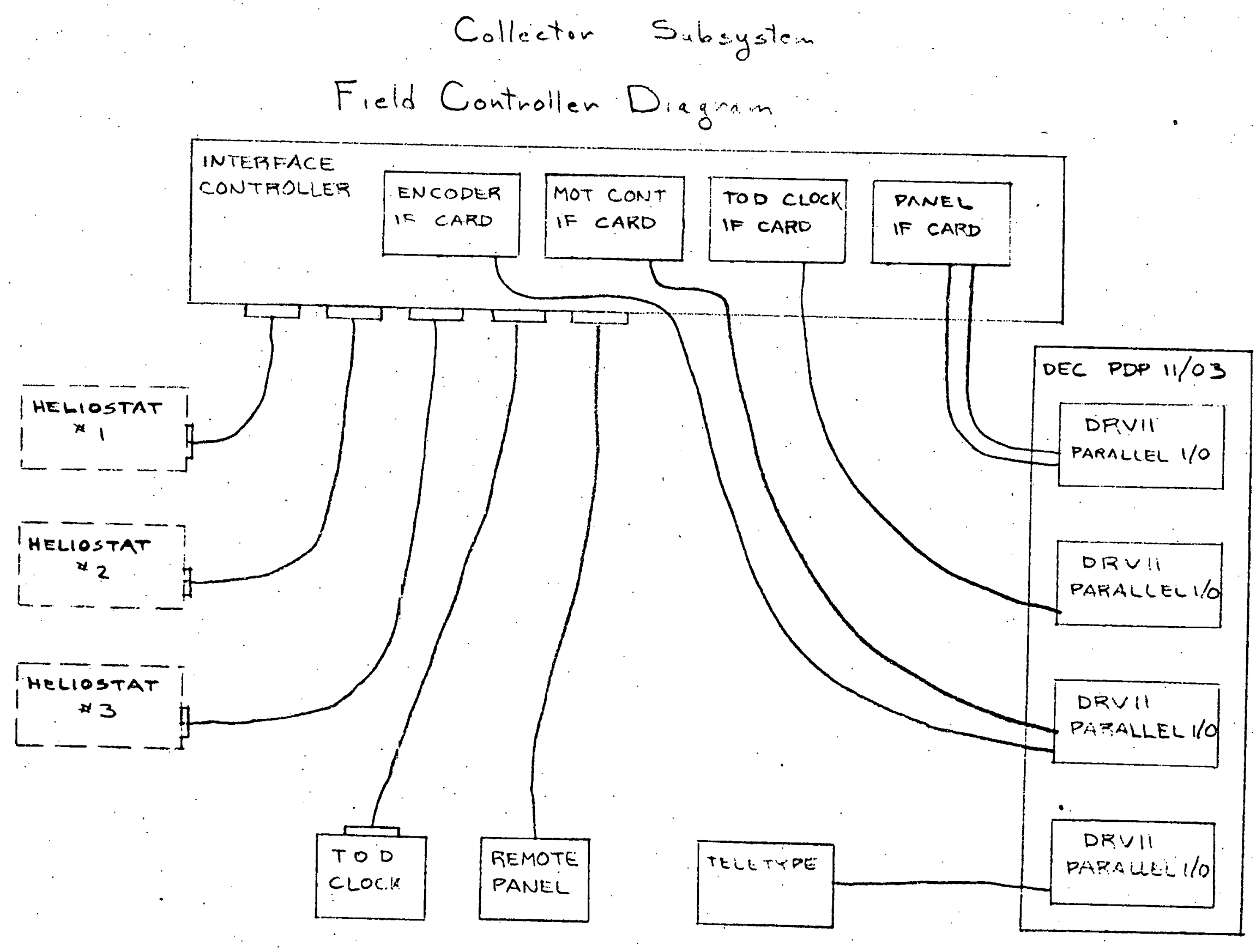




\section{Collector Subsystem}

\section{Drive and Control Assembly Error Budget}

\begin{tabular}{|l|l|l|l|l|}
\cline { 2 - 5 } \multicolumn{1}{c|}{} & $3 \sigma(99.73 \%)$ & $2 \sigma(95.4 \%)$ & $1 \sigma \quad(68.27 \%)$ & $0.67 \sigma \quad(50 \%)$ \\
\hline Reflector step tracking & $.01125^{\circ}$ & $.0107^{\circ}$ & $.0077^{\circ}$ & $.0056^{\circ}$ \\
Reflector step synchronization & $.01125^{\circ}$ & $.0107^{\circ}$ & $.0077^{\circ}$ & $.0056^{\circ}$ \\
Step response & $.0225^{\circ}$ & $.022^{\circ}$ & $.015^{\circ}$ & $.0112^{\circ}$ \\
Encoder & $.0178^{\circ}$ & $.0118^{\circ}$ & $.0059^{\circ}$ & $.004^{\circ}$ \\
Ephemeris data & $.009^{\circ}$ & $.006^{\circ}$ & $.003^{\circ}$ & $.002^{\circ}$ \\
Gimbal placement & $.045^{\circ}$ & $.030^{\circ}$ & $.015^{\circ}$ & $.010^{\circ}$ \\
Computer calculations, 16 bit & $.008^{\circ}$ & $.004^{\circ}$ & $.002^{\circ}$ & $.0014^{\circ}$ \\
Alignment (total, estimate) & $.1119^{\circ}$ & $.0746^{\circ}$ & $.0373^{\circ}$ & $.025^{\circ}$ \\
Drive actuation & $.108^{\circ}$ & $.072^{\circ}$ & $.036^{\circ}$ & $.024^{\circ}$ \\
Total (RSS) & $.165^{\circ}$ & $.112^{\circ}$ & $.057^{\circ}$ & $.038^{\circ}$ \\
\hline
\end{tabular}


SOFTWARE DEVELOPMENT CYCLE
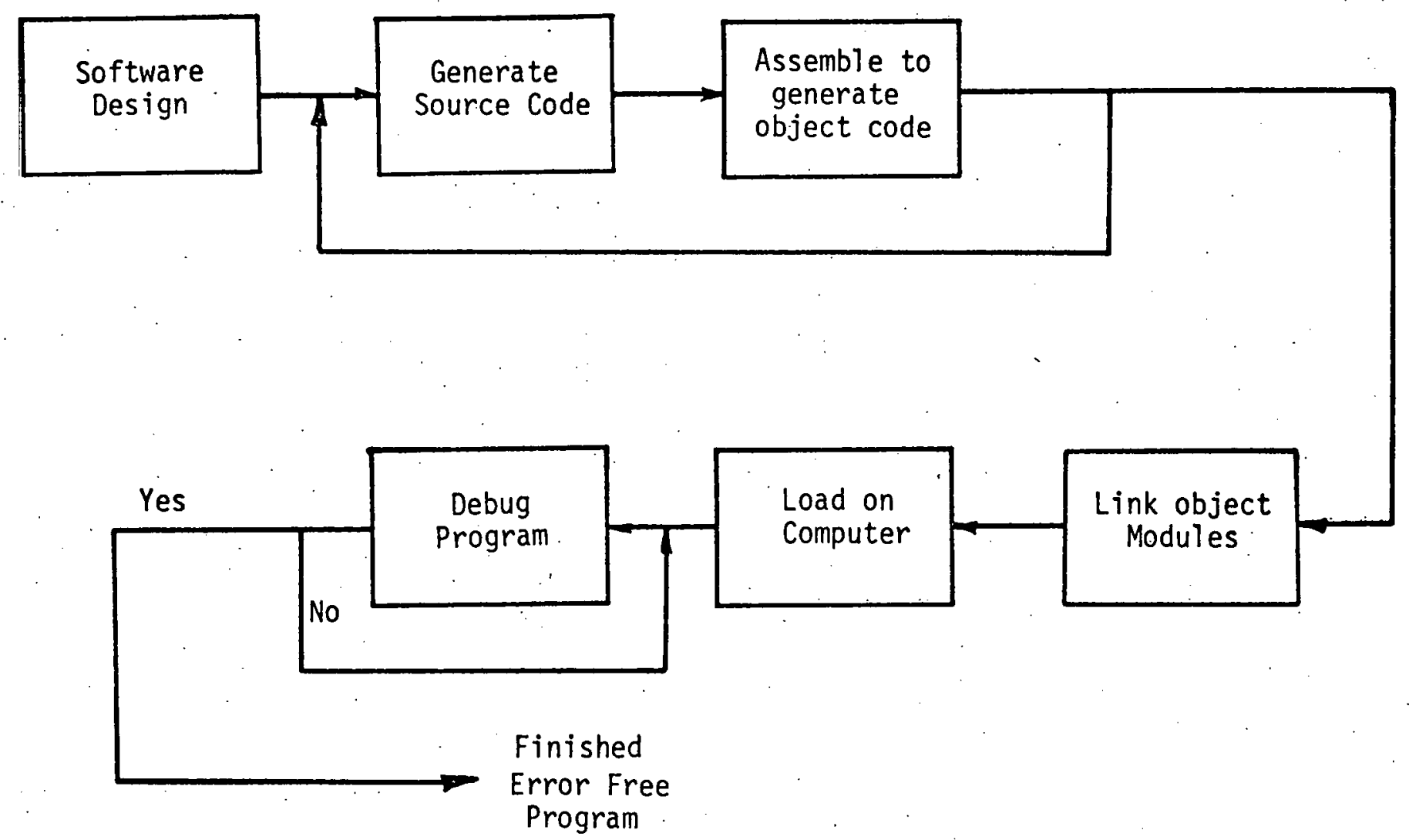
DRIVE AND CONTROL SOFTWARE FEATURES

- MODULARIZED SOFTWARE

- FIXED POINT CALCULATION

- 5 SEC update RATE 
Power-0n Routine

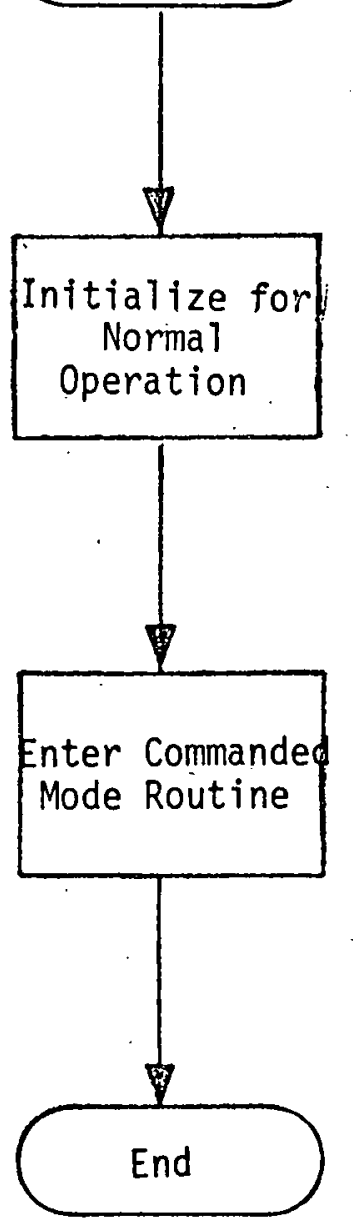

THE DODEOAOEFCOMPAN SEATTLE WASHINGTON 


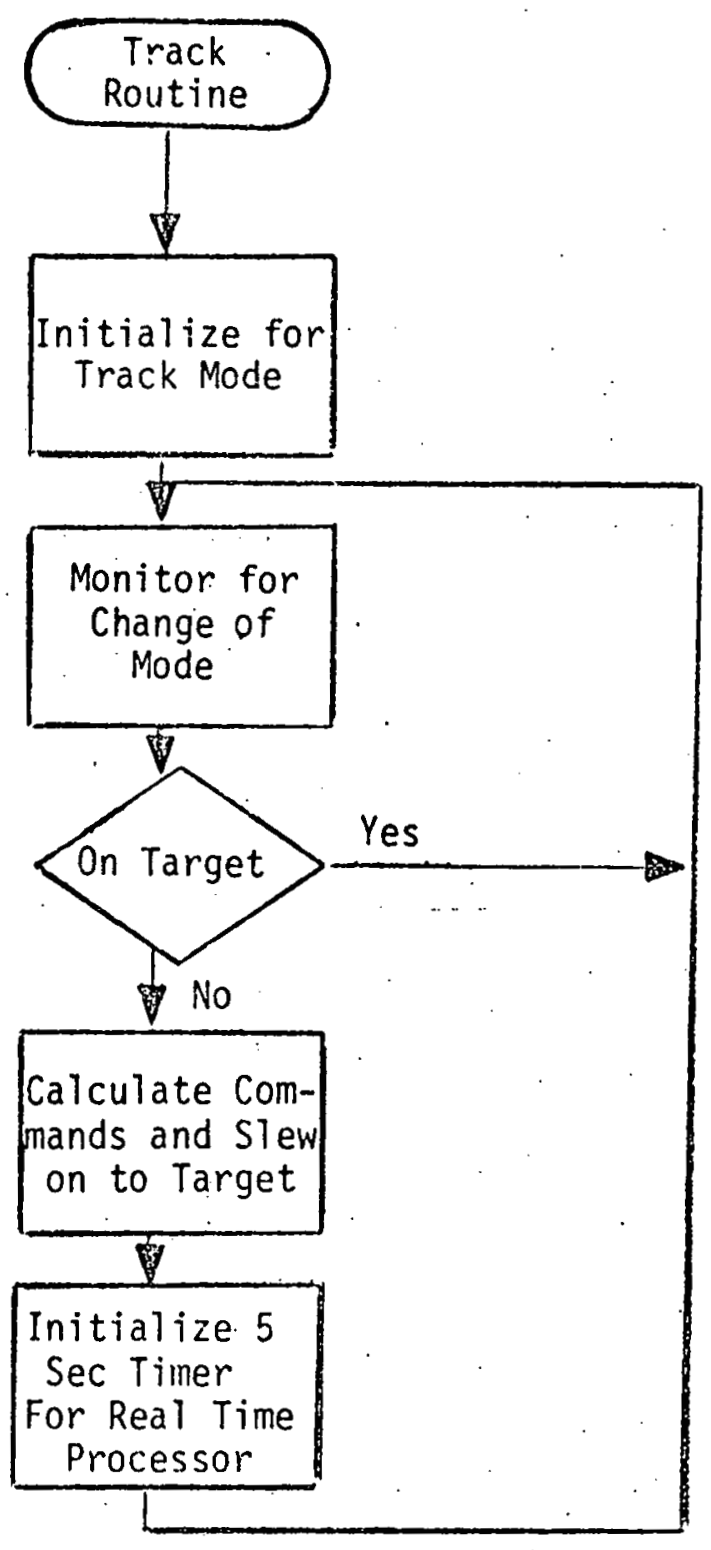

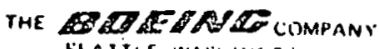




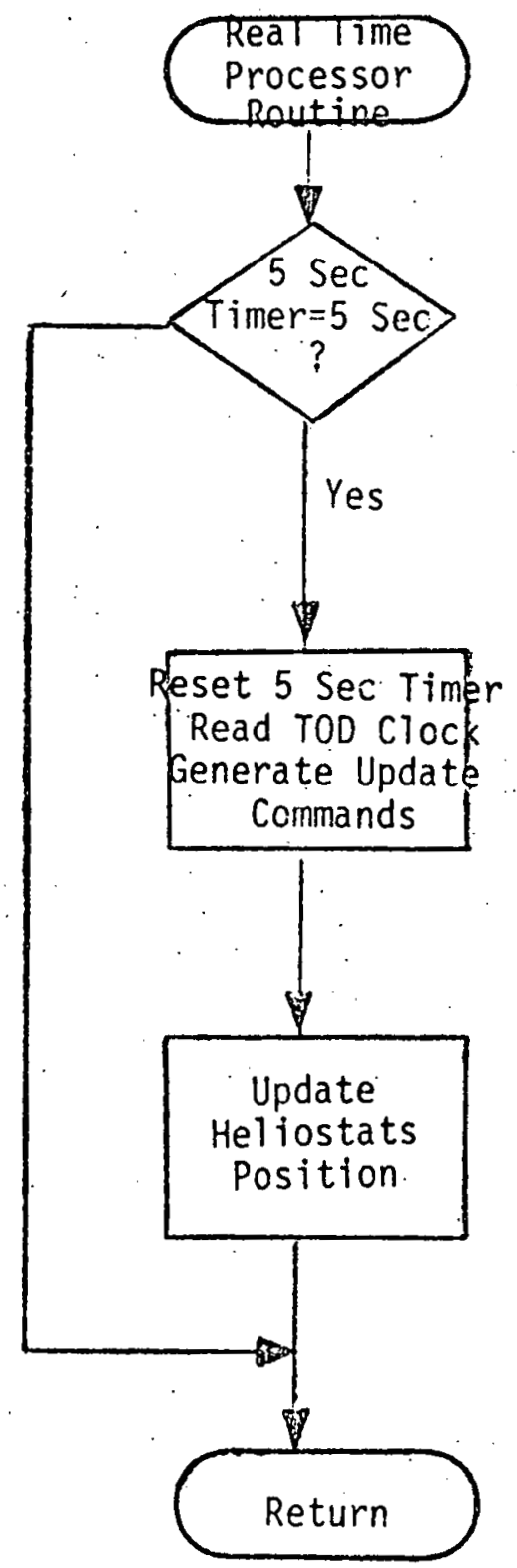

THE SBOESARDE COMPAN SHATtLE. WASHINGIONA 


\section{Geodolite Block Diagram}

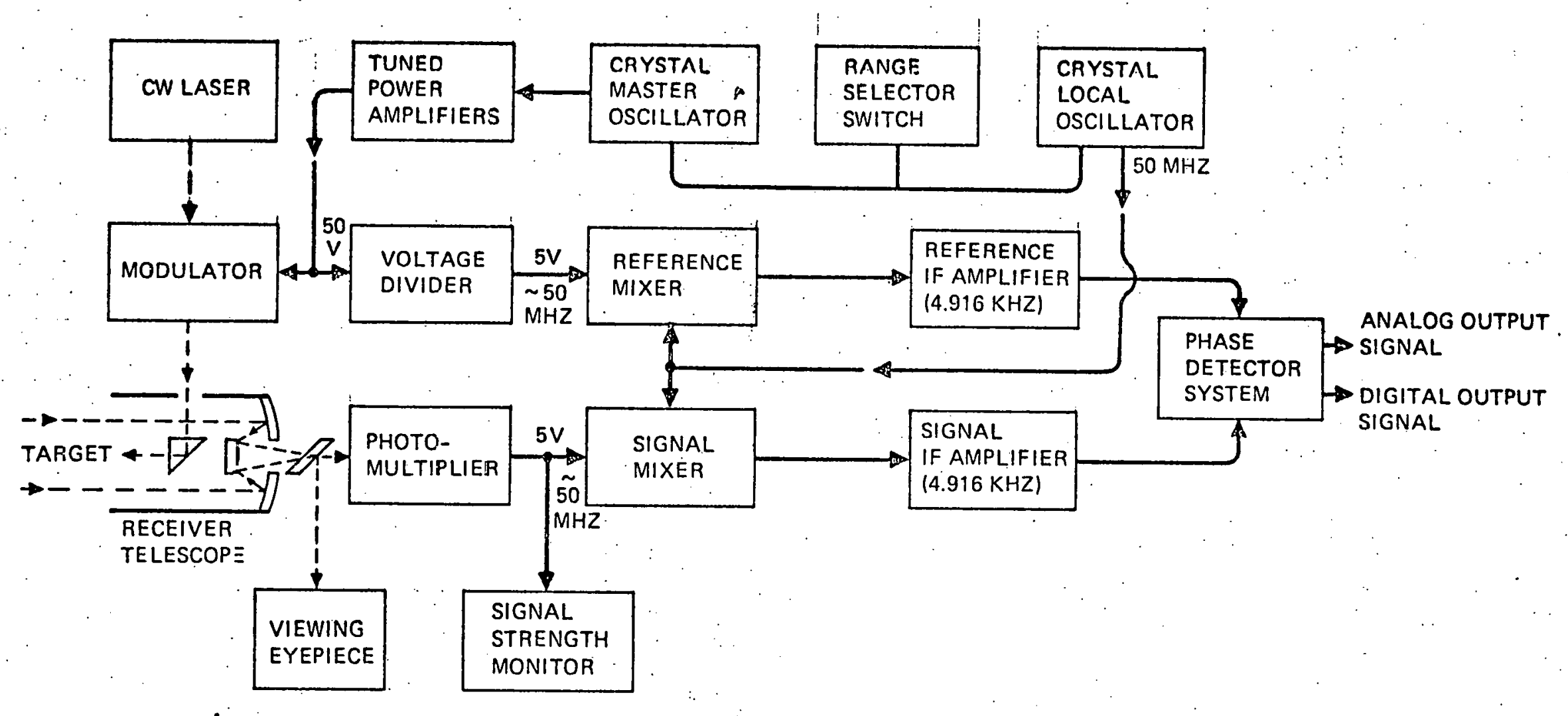




\section{Alignment Procedure}
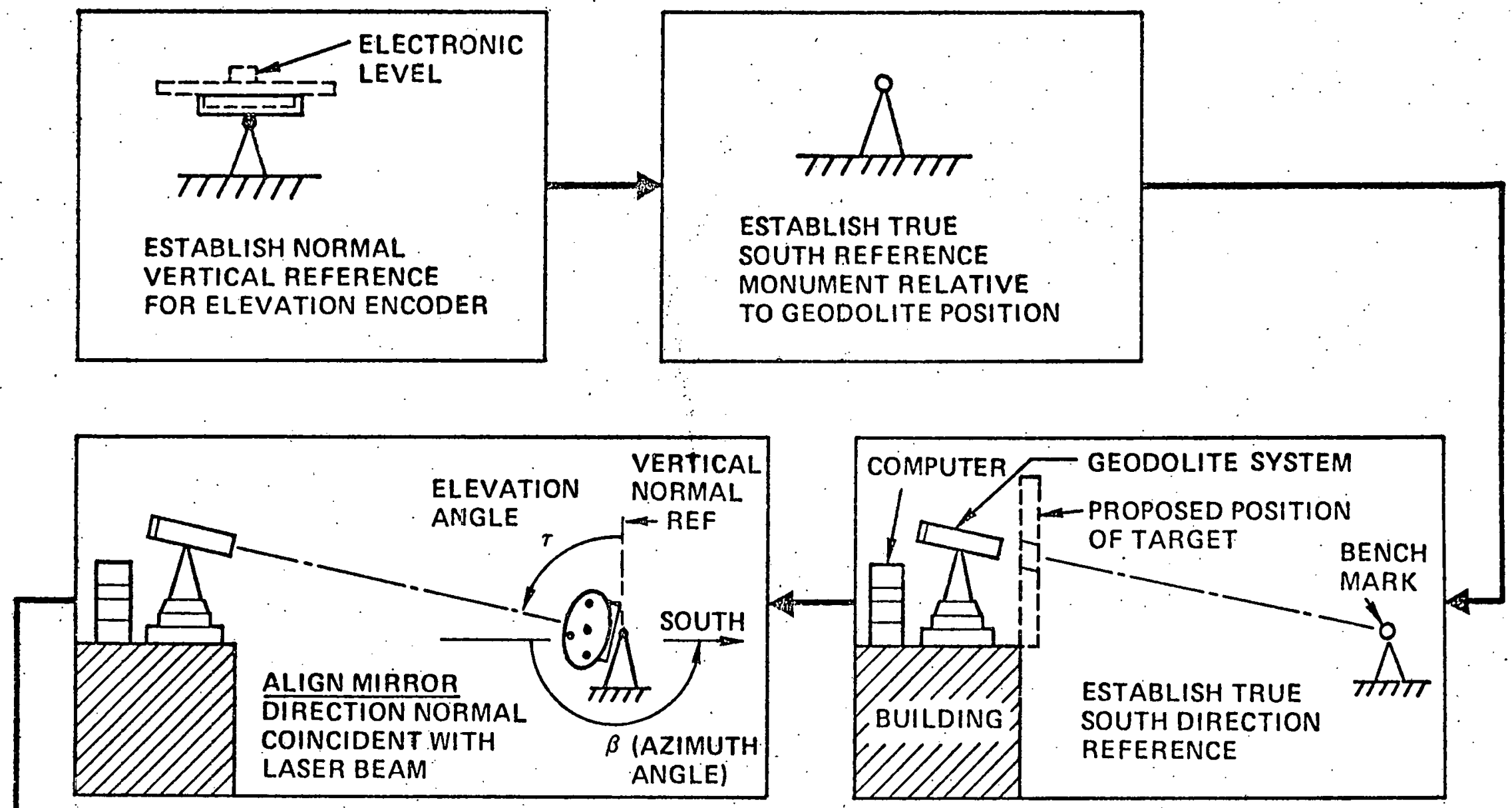

UP-DATE MASTER CONTROLLLER

WITH ENCODER POSITIONS 
COLLECTOR SUBSYSTEM MANUFACTURING

- TRANSPARENT ENCLOSURE

- REFLECTOR 


\section{Transparent Enclosure Fabrication Steps}

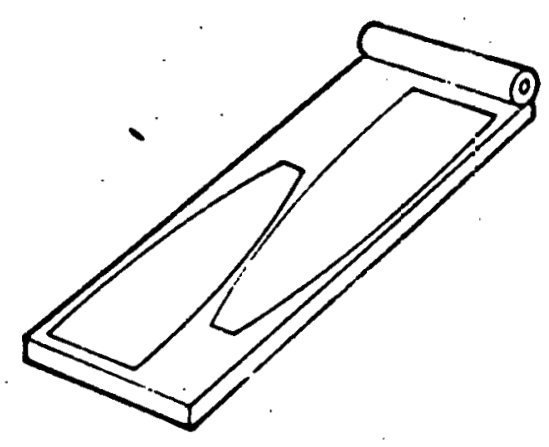

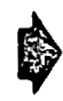

CUTOUT GORES
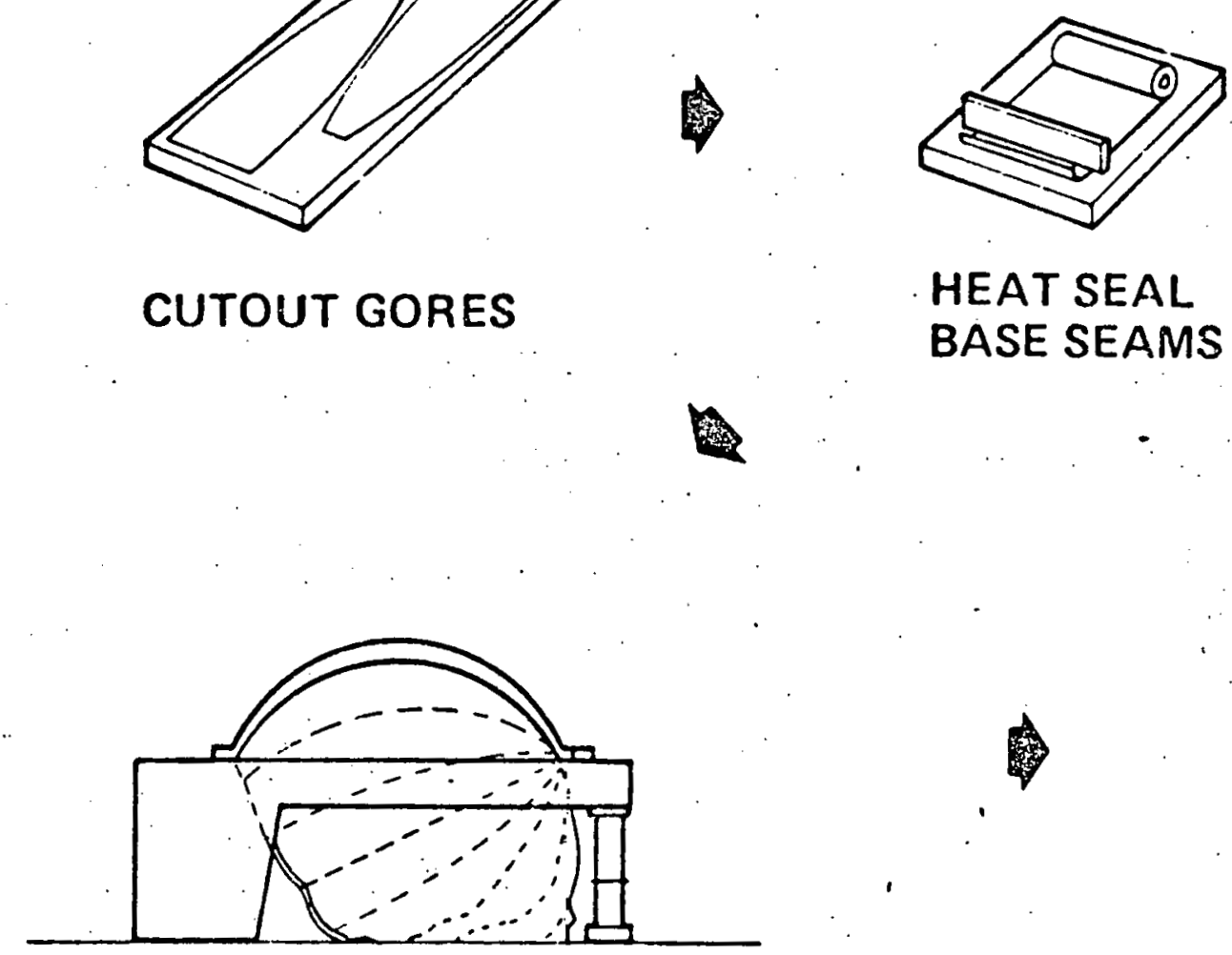

HEAT SEAL BASE SEAMS

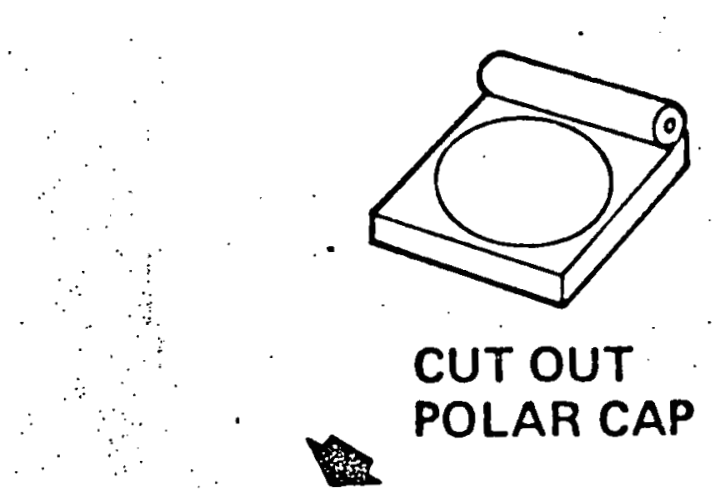

HEAT SEAL GORES

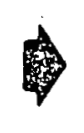

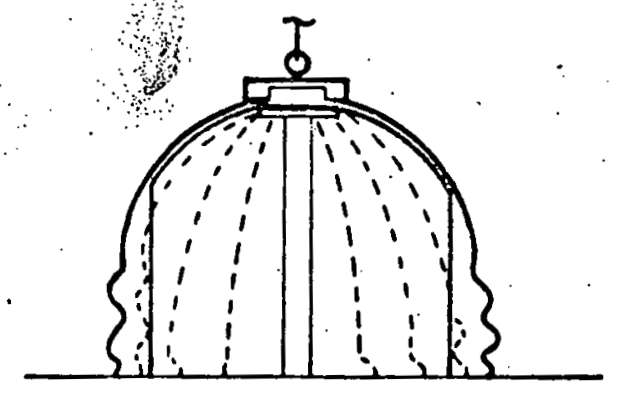

HEAT SEAL POLAR CAP 


\section{DOME SEAM FABRICATION}

TEDLAR HEAT SEAL REQUIREMENTS

- HEAT TO 205 TO $218^{\circ} \mathrm{C}\left(400\right.$ TO $425^{\circ} \mathrm{F}$ )

0 RESTRAINT PRESSURE OF 20 PSI DURING HEATING AND COOLING

PROVEN HEAT SEAL'TECHNIQUES

ULTRASONIC - HEAT GENERATED BY HORN VIBRATING AT 20,000 HERTZ

- PRESSURE IS LOCALIZED ON HORN

IMPULSE- - HEAT GENERATED BY RESISTANCE WIRE

- PRESSURE REQUIRED FOR FULLL LENGTH OF JOINT 


\section{ULTRASONIC HEAT SEALING}

\section{PROCESS DEVELOPMENTS}

- CAN MAKE CONTINUOUS SEAL

- CAN MAKE SFOT WELDS

- CAN BE MANIPULATED TO MAKE CLOSE-OUT JOINTS

- HAVE MADE SLBSCALE DOME (42". DIAMETER)

CRITICAL REQUIFEMENTS

0 DISTANCE BETWEEN HORN AND ANVIL: TOLERANCE OF \pm .0012 INCHES

- UNIFORM SPEED FOR GIVEN MACHINE SETTING

JOINT CHARACTERISTICS

- STRENGTH EXCEEDS YIELD STRENGTH OF FILM BY 35 PERCENT 


\section{Ultrasonic Sealing of Tedlar Dome Material}

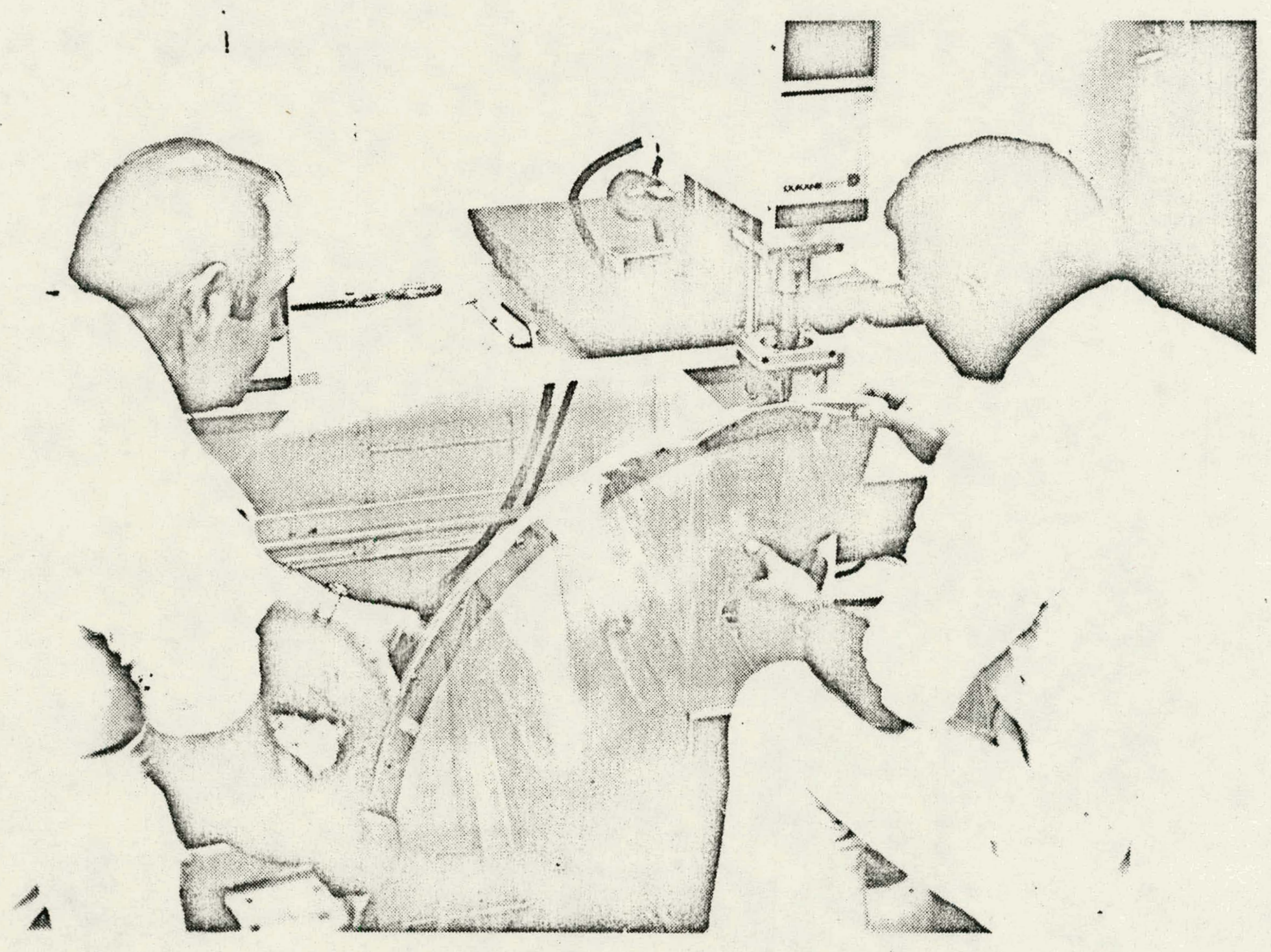


IMPULSE HEAT SEALING

PROCESS DEVELOPMENTS

- CAN MAKE LONG, CURVED SEAMS

- CAN SEAL FOUR LAYERS

- CAN OVERLAP SEAL

CRITICAL REQUIREMENTS

- SUFFICIENT ENERGY PULSE TO OBTAIN FILM TEMPERATURE

- RESTRAINT ON FILM TO PREVENT SHRINKAGE

JOINT CHARACTERISTICS

0 STRENGTH EXCEEDS YIELD STRENGTH OF FILM BY 36 PERCENT 

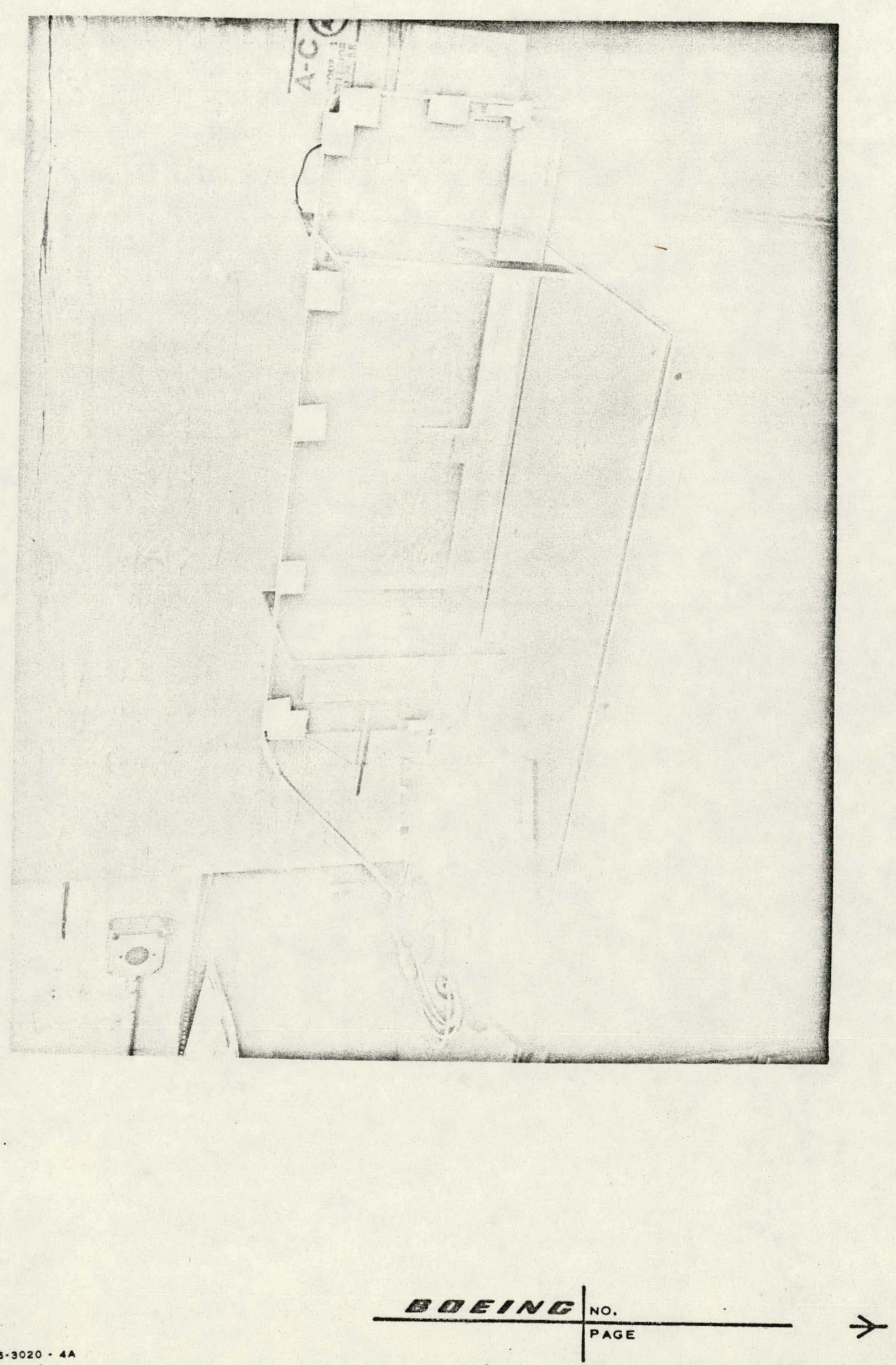
:
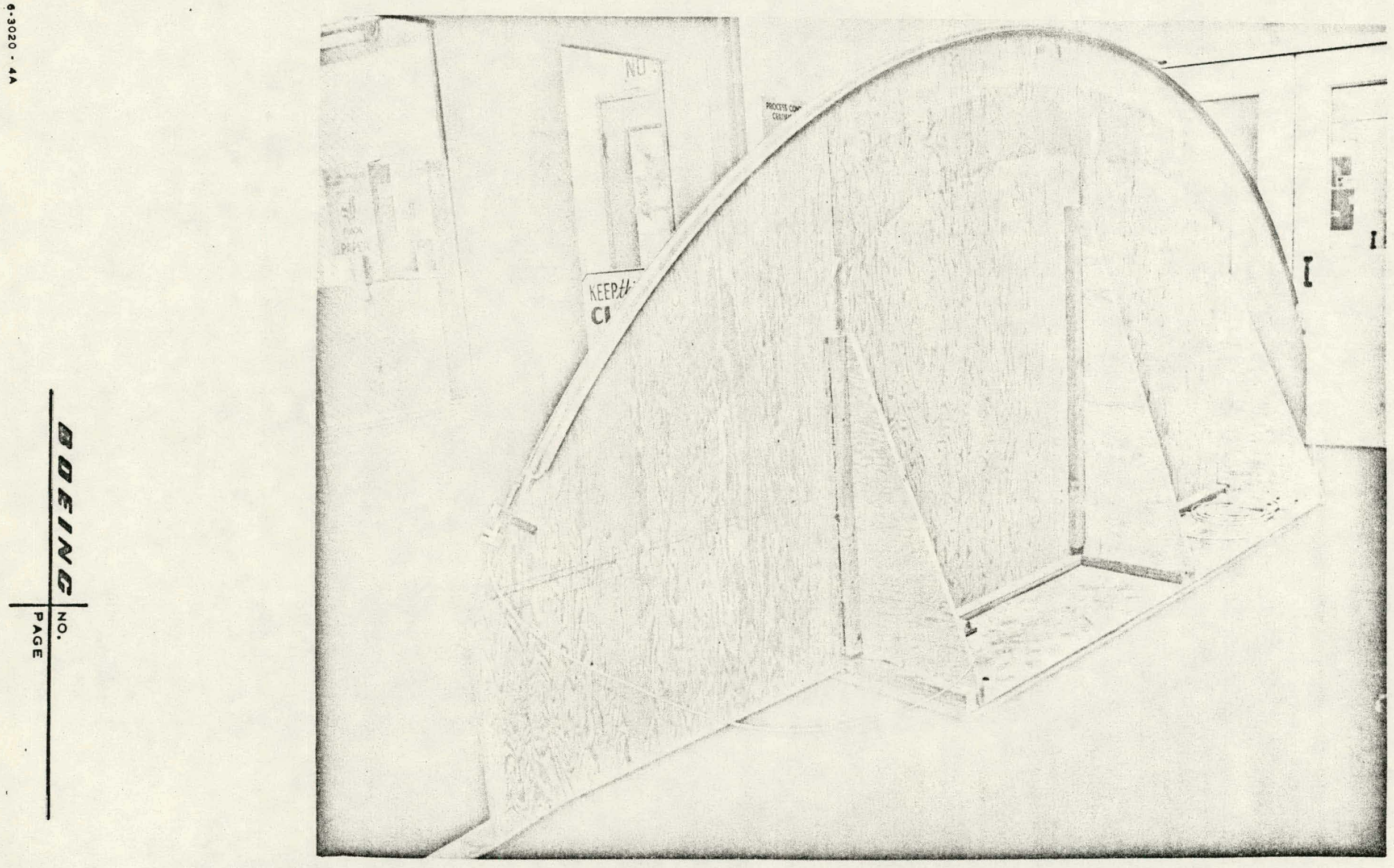

$\psi$ 



\section{THERMOFORMING POLISHED TEDLAR}

- POLISHING CREATES NON-UNIFORM HEAT SHRINK

- PRETENSIONING REQUIRED DURING THERMOFORMING

- COST EFFECTIVE TO USE 18 GORES

- THERMOFORMING NOT REQUIRED (LESS THAN 1.5\% OUT OF ROUND)

- MORE EFFECTIVE USE OF TEDLOR FILM (LESS SCRAP) 
ENCLOSURE TOOLING

\author{
MAJOR TOOLS - GORE SEAM FABRICATION \\ - POLAR CAP SEAM FABRICATION \\ OTHERS - GORE TEMPLATE \\ - polar cap template \\ - BASE SEAM FABRICATION \\ - STORAGE AND LAYOUT
}




\section{Gore Seam Tool Concept}

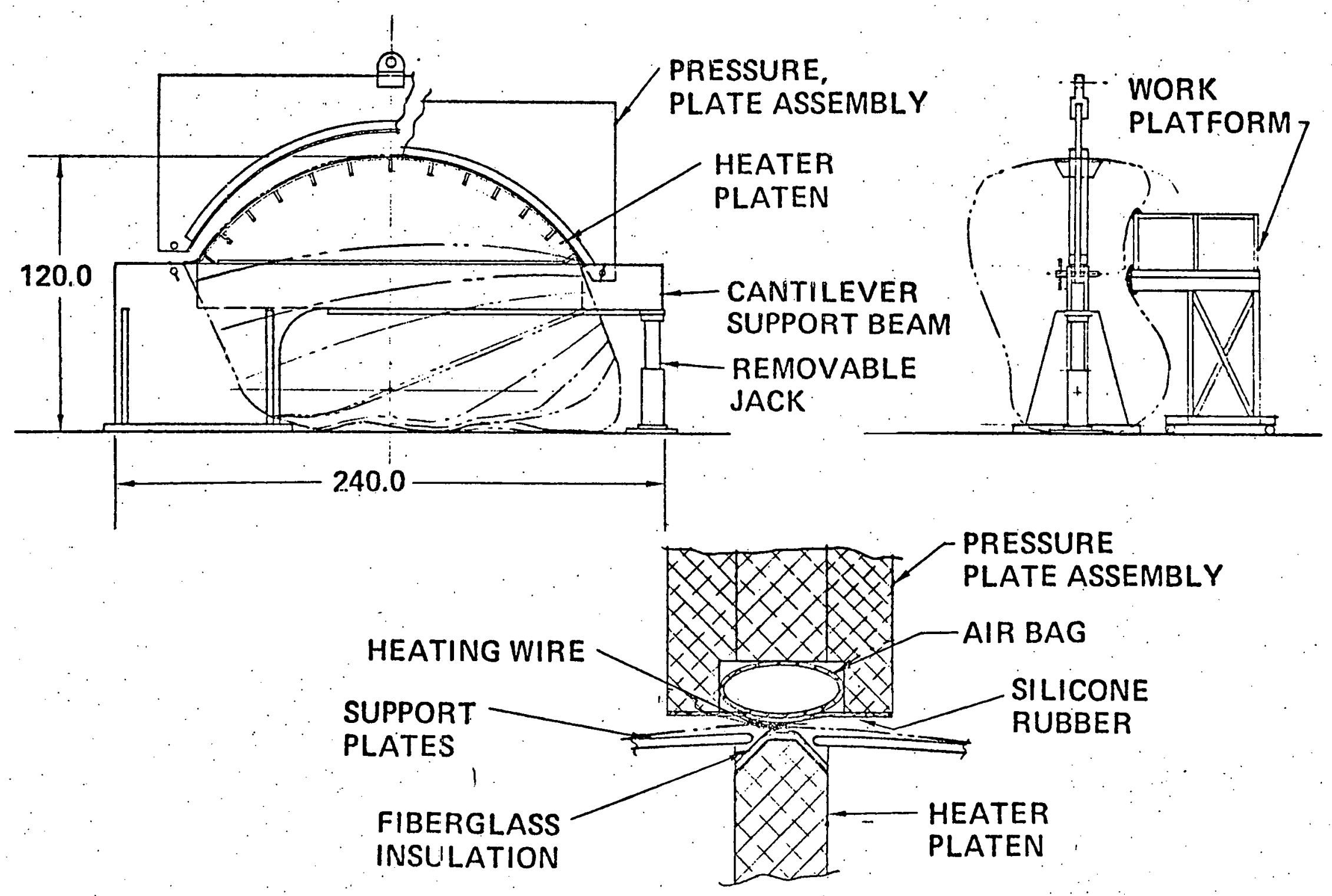




\section{Polar Cap Seam}

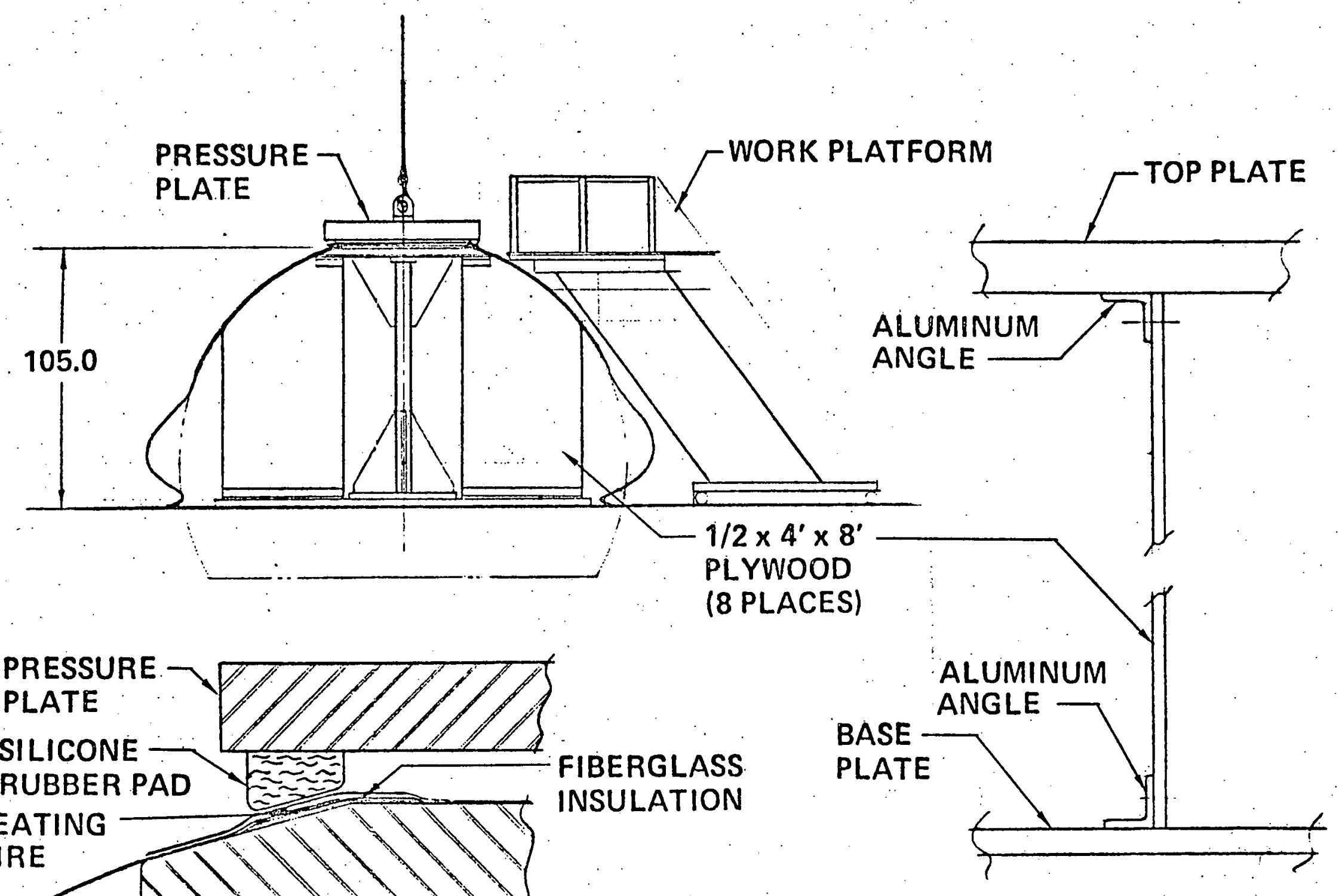




\section{Reflector Fabrication Steps}

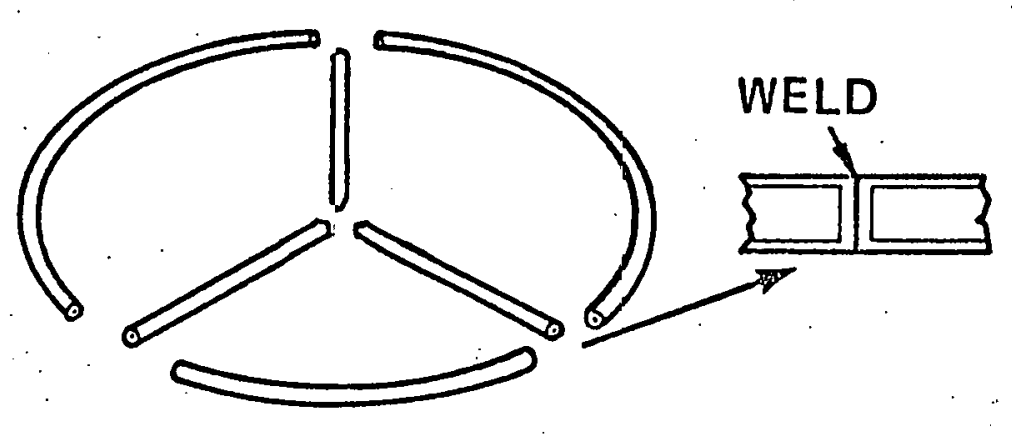

ROLL RING AND WELD SEGMENTS

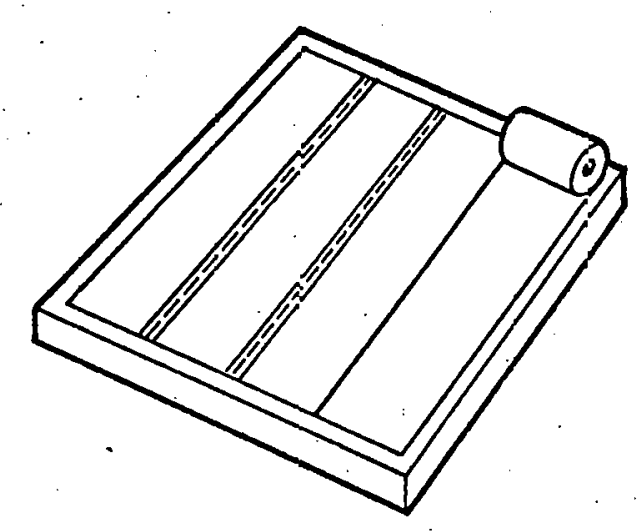

BOND MYLAR SEAMS

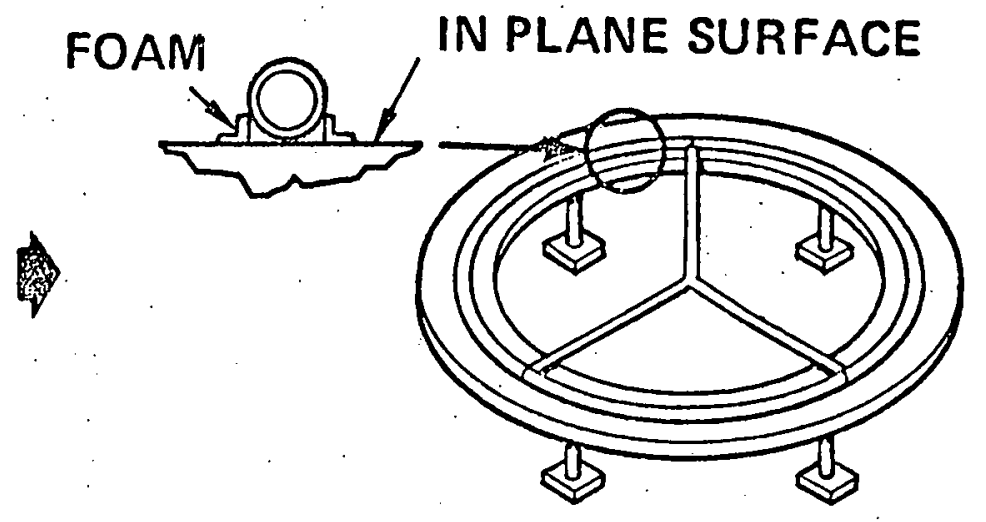

FOAM FLAT SURFACE

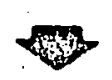

筍

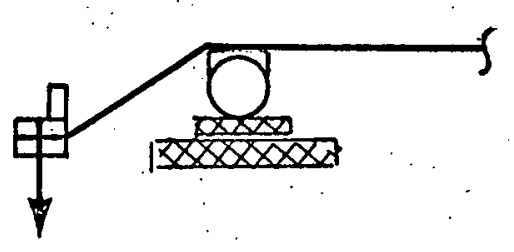

TENSIONING AND BONDING MYLAR 
REFLECTOR PROCESS DEVELOPMENT .

\section{REFLECTOR SEAMS}

- ELIMINATE MARK-OFF AND RUN-OUT BY ADHESIVE SELECTION AND APPLICATION CONTROL

- PRESSURE DURING CURE ELIMINATES HEAT TREATING

FOAMED SURFACE

- SELF-SKINNING URETHANE FOAM PROVIDES:

- SATISFACTORY SURFACE

- SUFFICIENT STRENGTH

VERIFICATION REFLECTORS

- FABRICATED 52 INCH AND 36 INCH REFLECTORS 
MYLAR TO MYLAR BOND

ADHESIVE - CURED POLYESTER

FORM - SOLVENT DISPENSION

AMOUNT - DRY FILM THICKNESS OF, 2 TO ,3 MILS

CURE - HEAT TO $275^{\circ} \mathrm{F}$ TO $325^{\circ} \mathrm{F}$ WHILE UNDER PRESSURE (10 PSI MIN) 


\section{SELF-SKINNING PLASTIC FOAM}

MATERIAL - TWO PART URETHANE FOAM (COROFOAM 586/222)

DENSITY - CONSTRAINED TO 15 LBS/FT 3

CURE - DEMOLD TIME - 30 MINUTES

POST CURE 16 HOURS AT RT

ACCELERATED CURE - 1 HOUR AT $150^{\circ} \mathrm{F}$ 
MYLAR TO FOAM BOND

MATERIAL - URETHANE 2 PART ADHESIVE

FORM $\quad-100$ PERCENT SOLIDS

APPLICATION - APPLY RELATIVE THICK (.032 IN MAX)

LAYER TO FOAM

CURE $\quad-24$ HOURS AT R.T.

- 60 MINUTES AT $150^{\circ} \mathrm{F}$ 


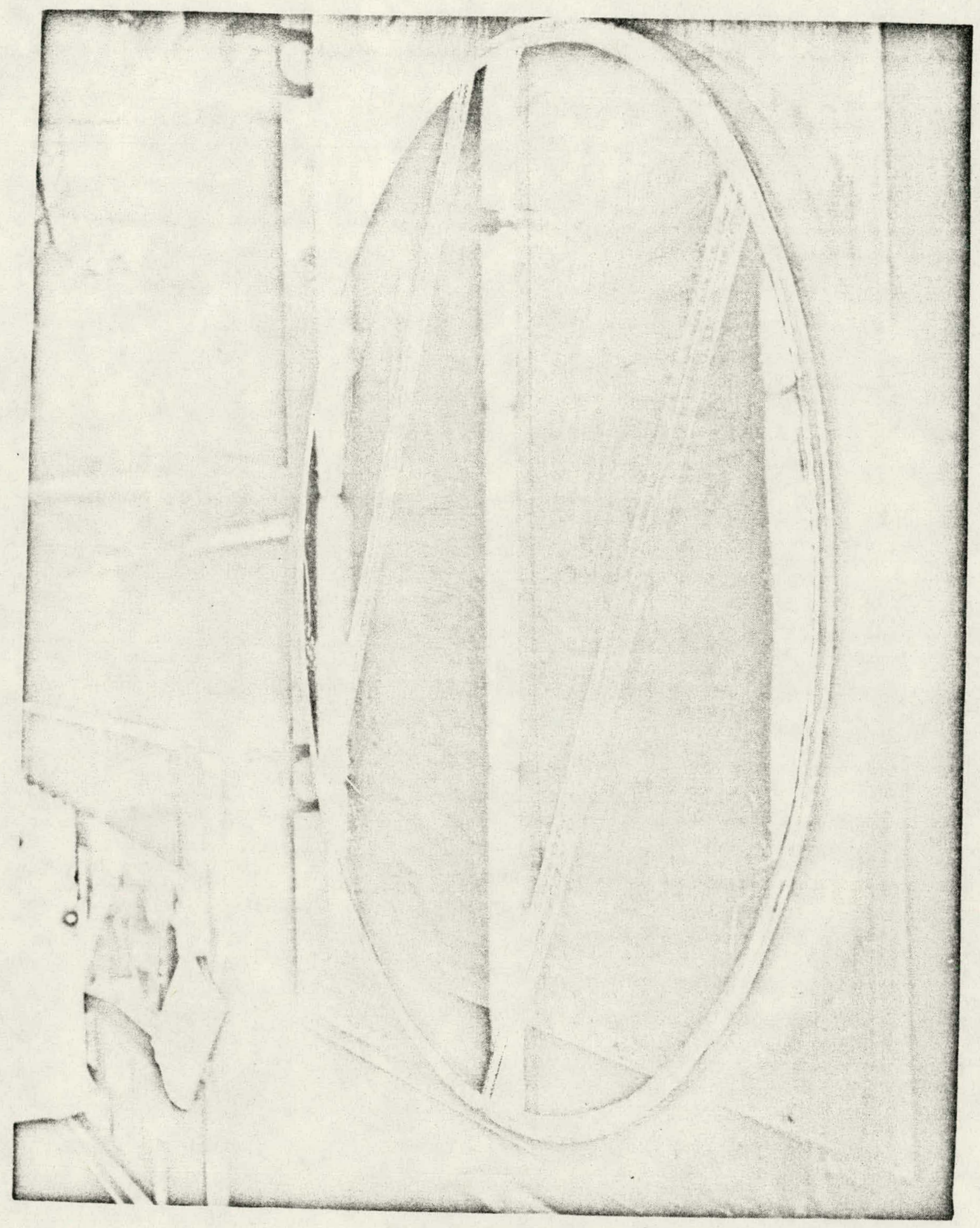


REFLECTOR TOOLING

MAJOR TOOL - FOAMING AND TENSIONING TOOL
OTHERS - ROLLED RING TEMPLATE
- WELDING JIG
- LAYOUT AND STORAGE. 
Foaming and Tensioning

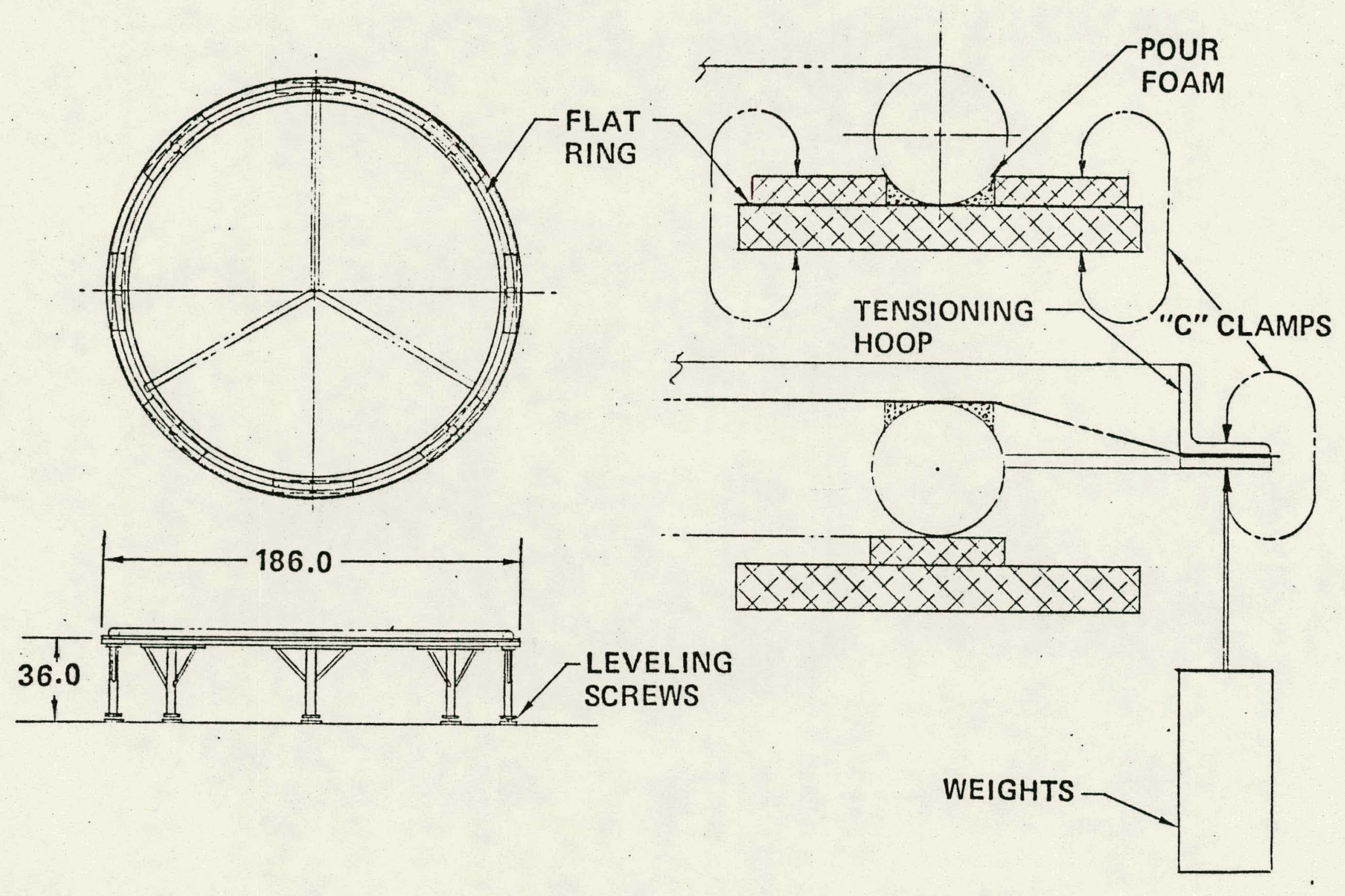




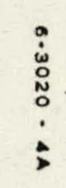

$\mid$

$\psi$

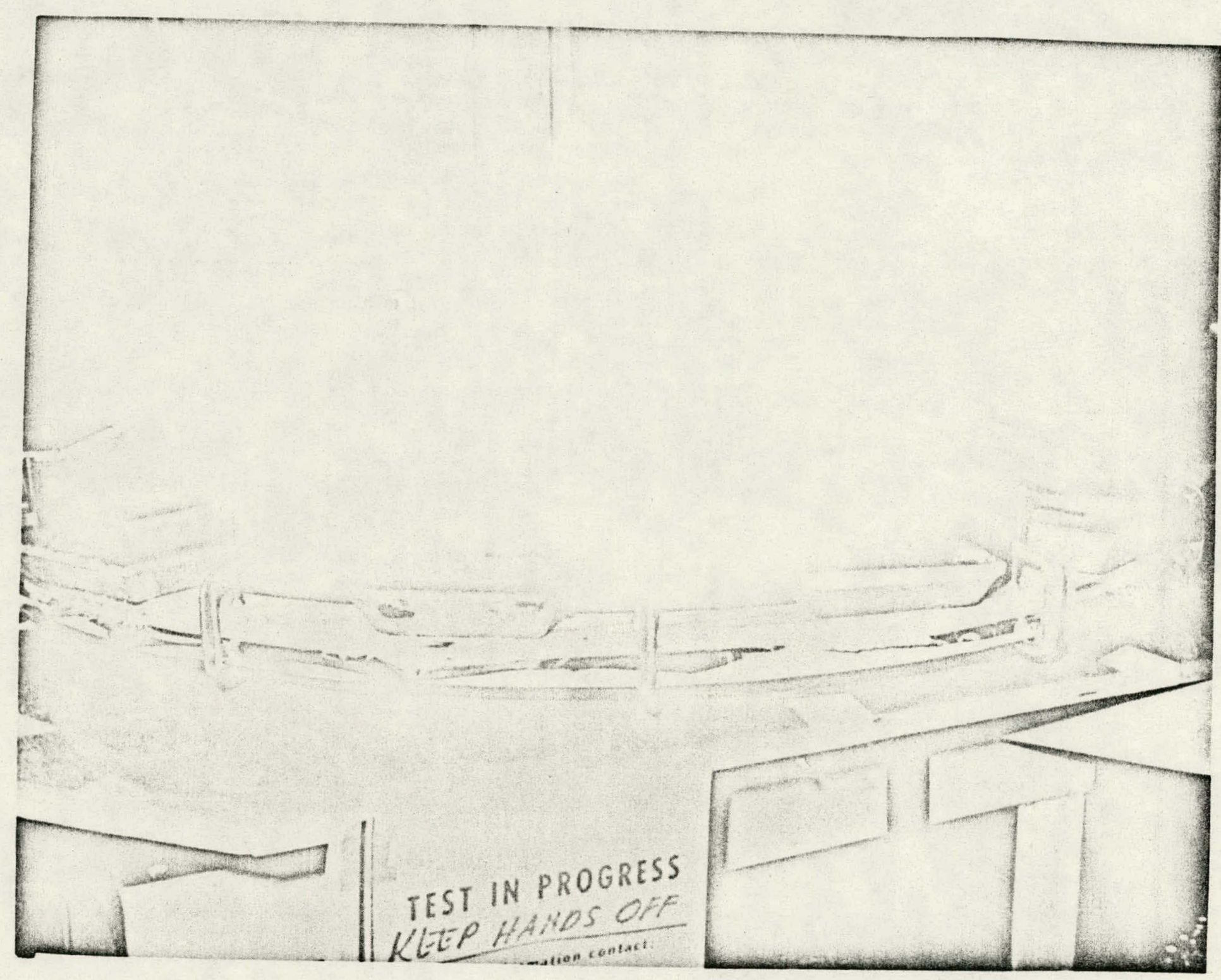




\title{
CONCLUSIONS
}

MANUFACTURING PROCESSES HAVE BEEN

\author{
- SELECTED \\ - DOCUMENTED \\ HAVE VERIFIED FEASIBILITY \\ WE ARE READY FOR PRODUCTION
}




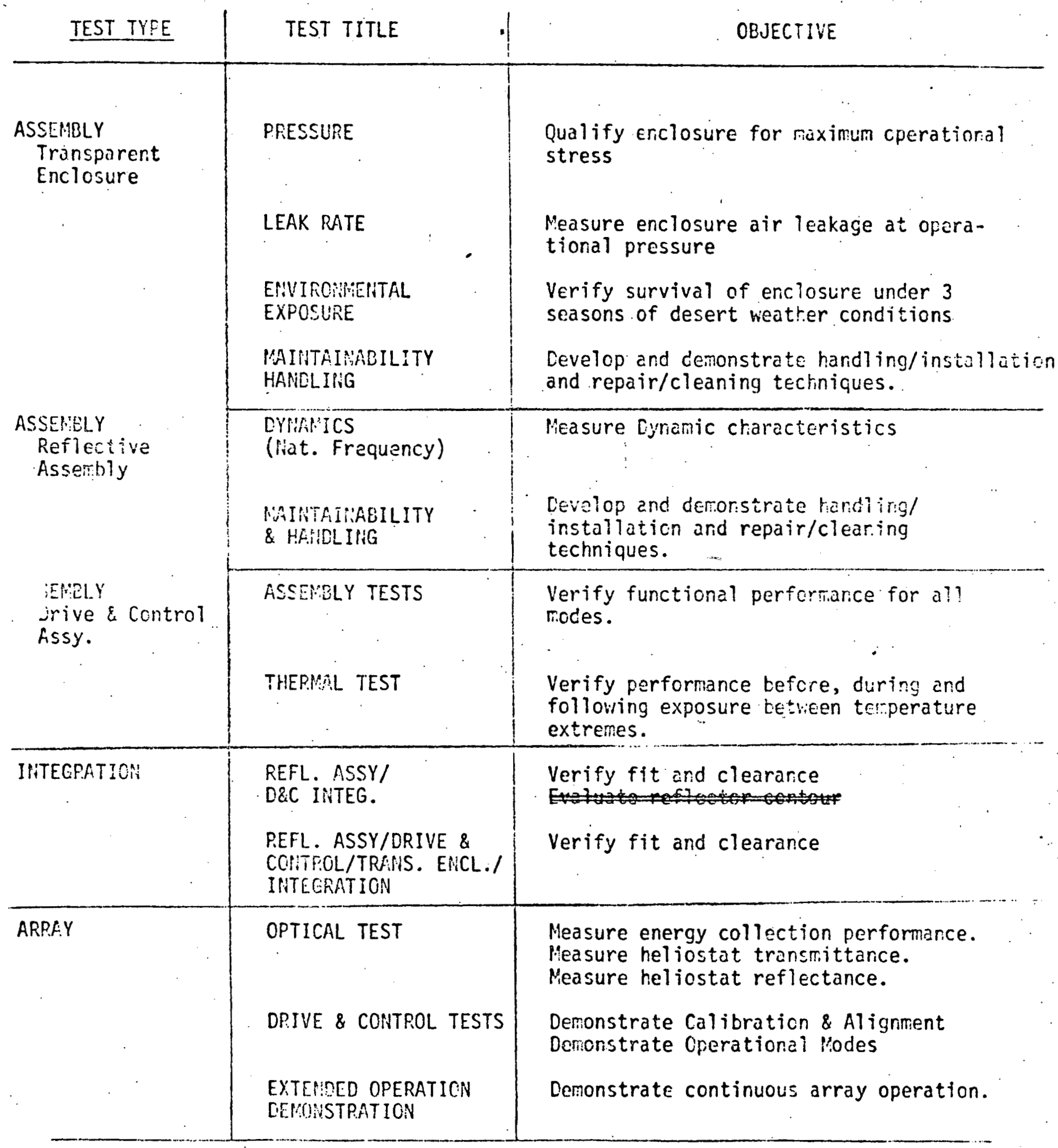




\section{Pressure and Leak Rate Test}
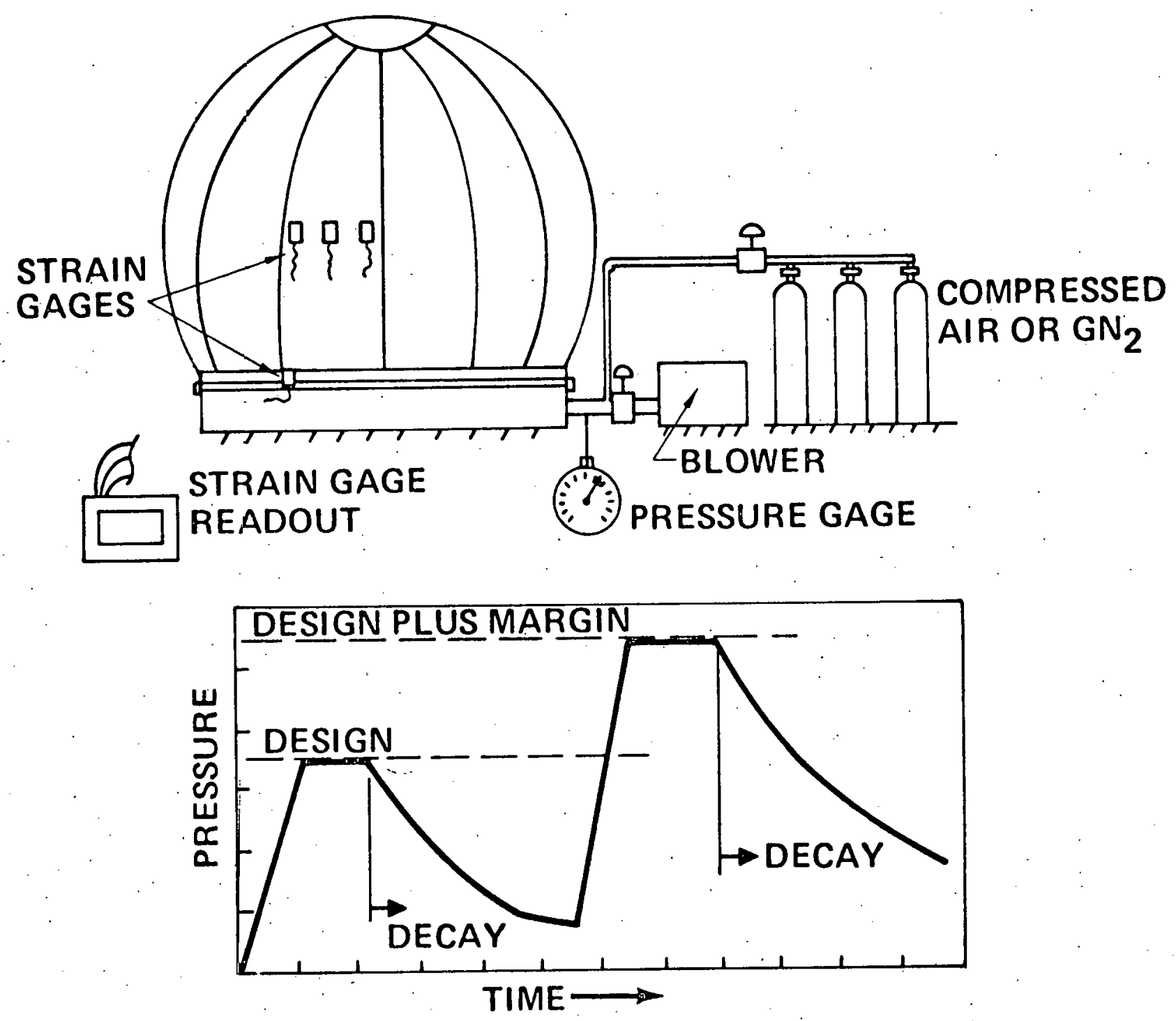


\section{Environmental Exposure-Boardman Site}

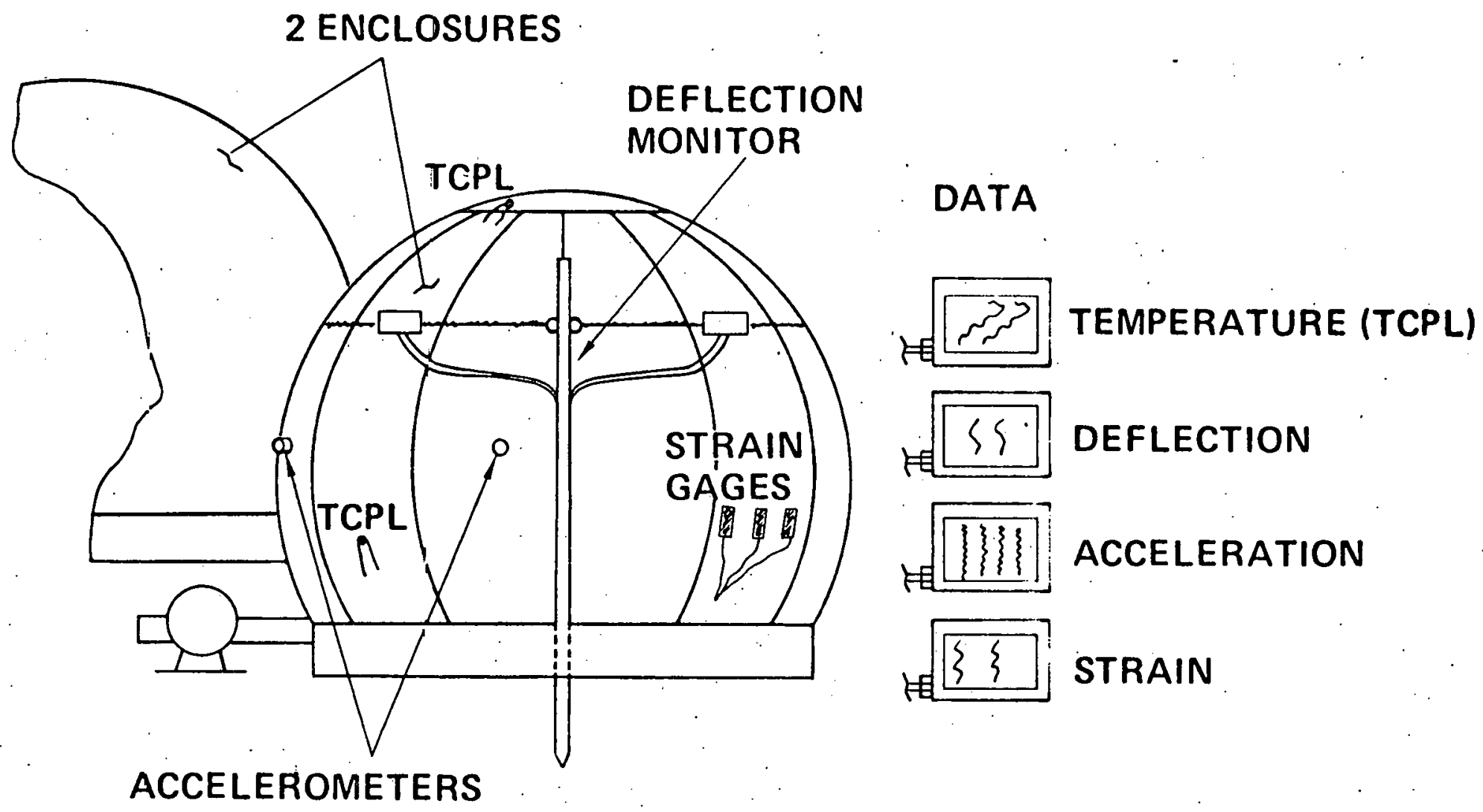




\section{Dynamics Test}

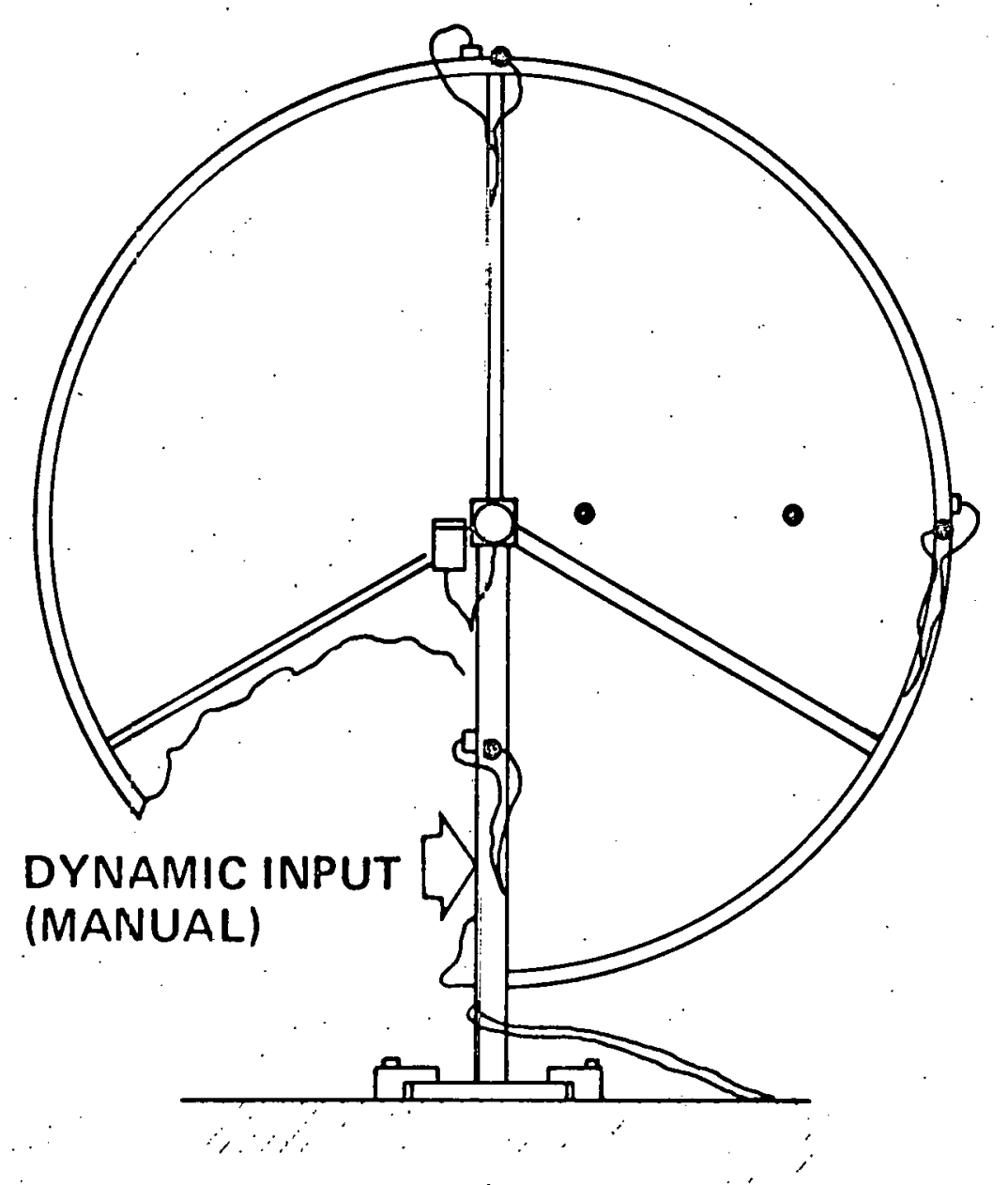

ACCELEROMETER READOUT EQUIPMENT

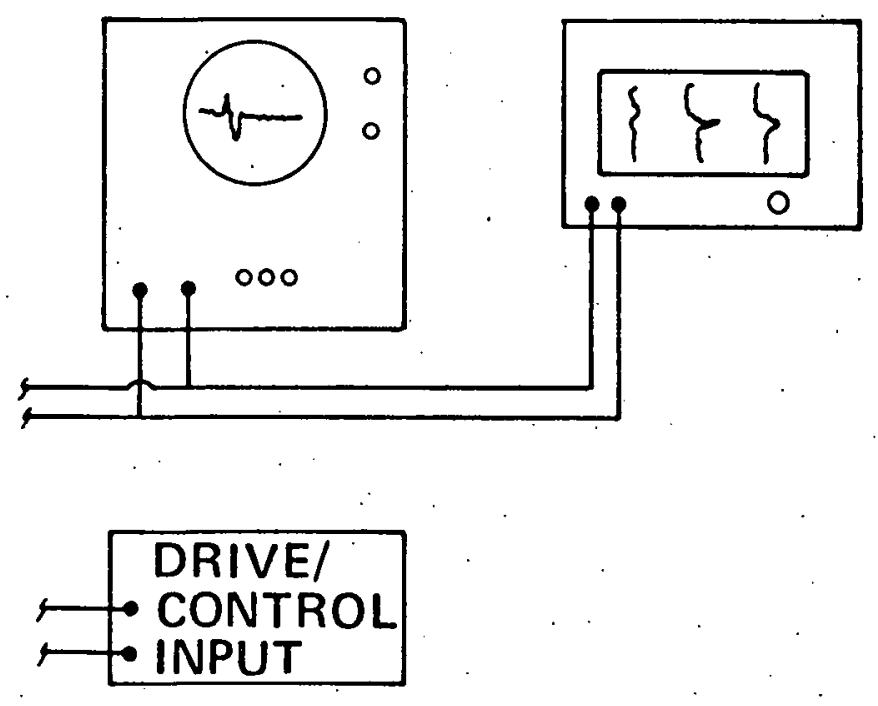




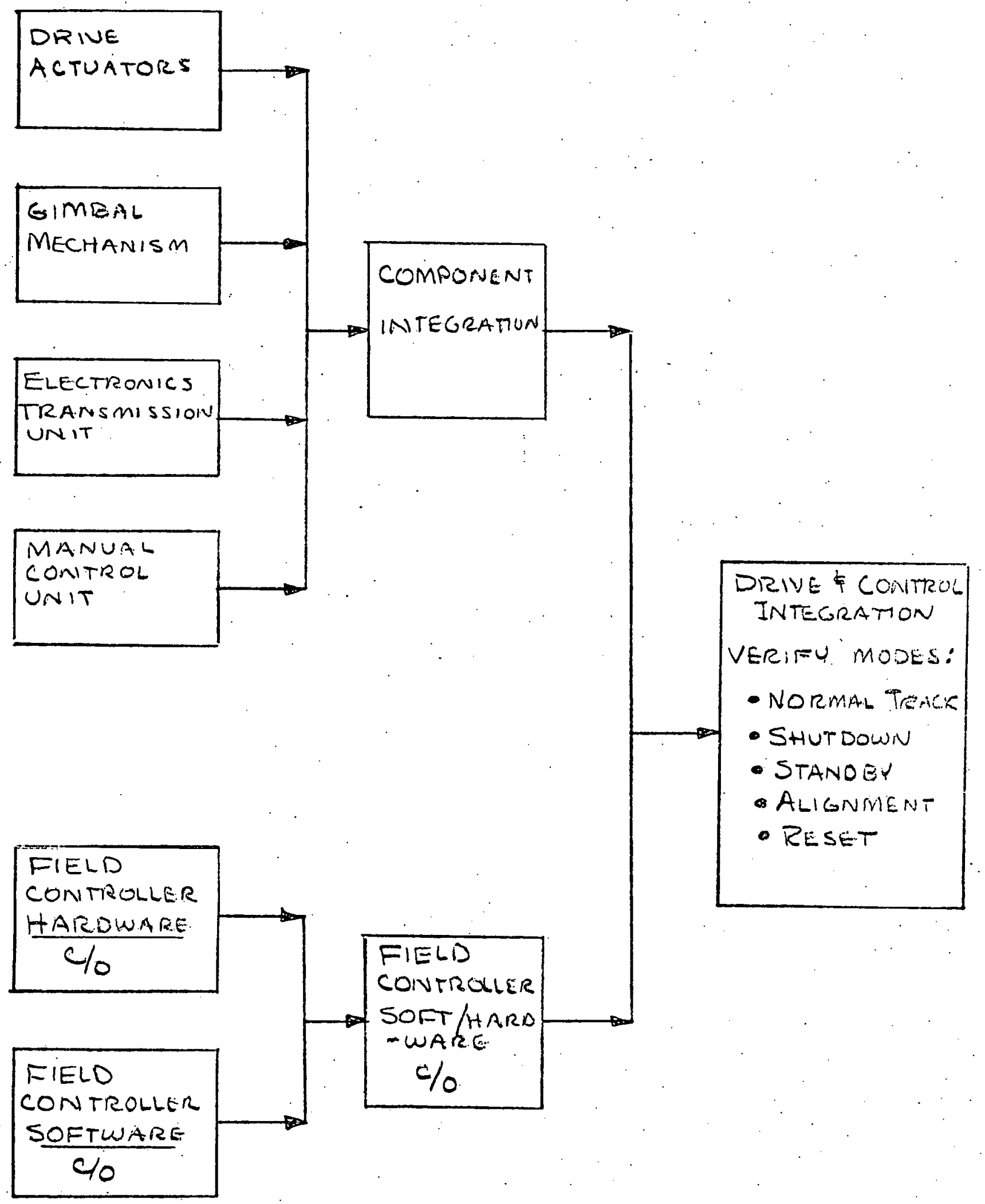

Drive \& control Assembly integration 
- Verify interfaces

- verify fit $\frac{1}{\text { clearanices }}$

- functional performance

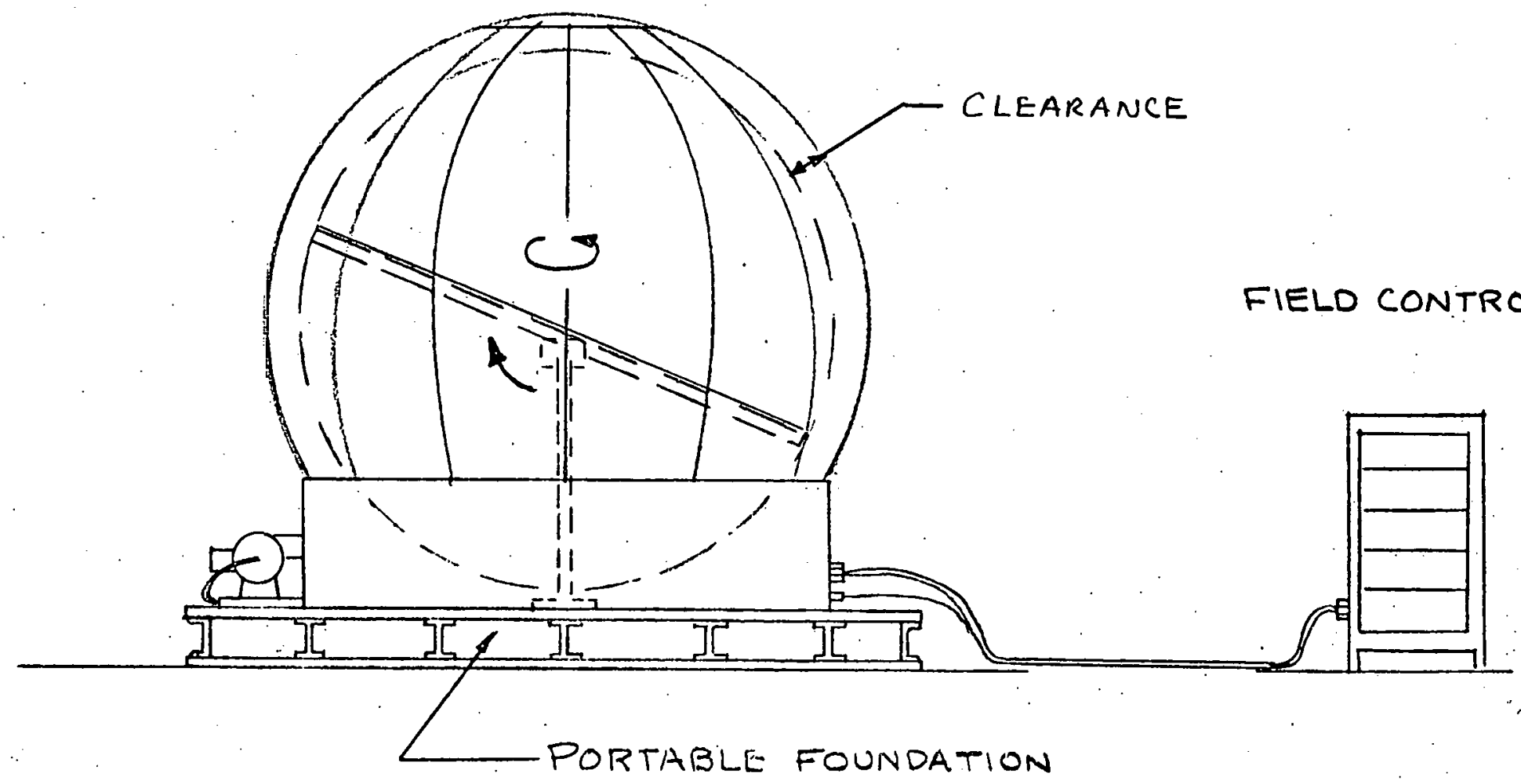




\section{Heliostat Array Test-Plan View}

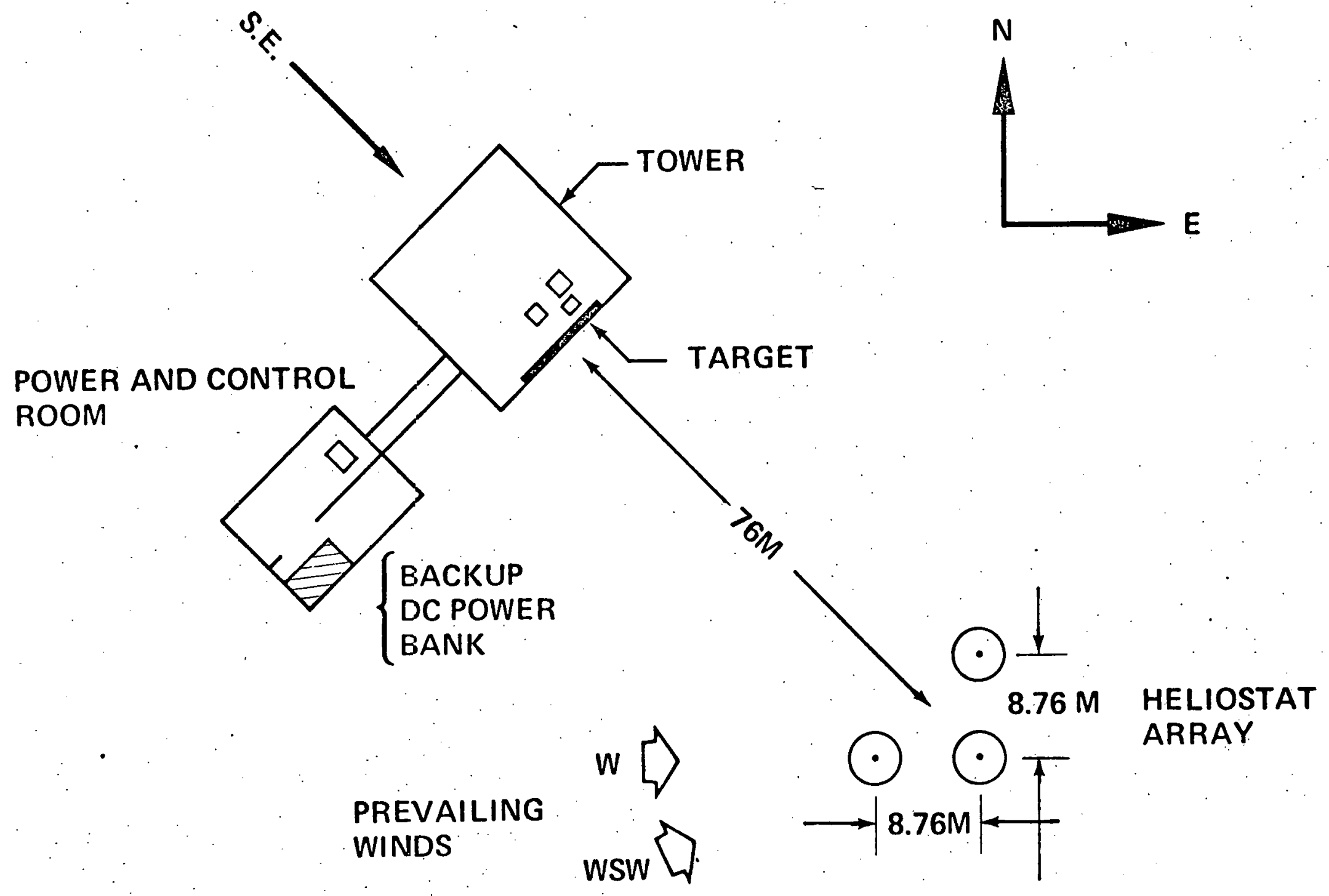




\section{Heliostat Array Test-Elev. View}

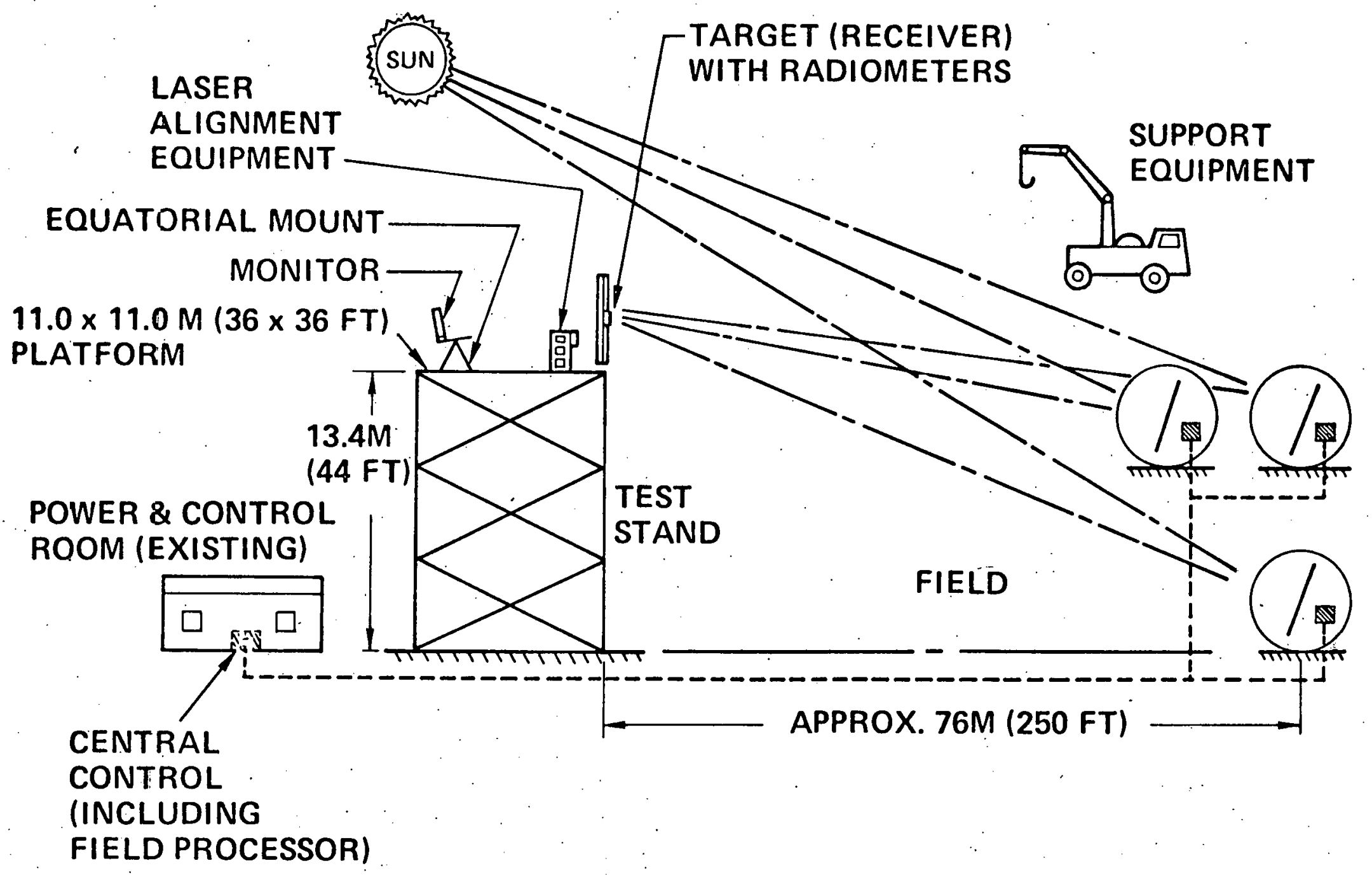




\section{Dome Transmittance Test}

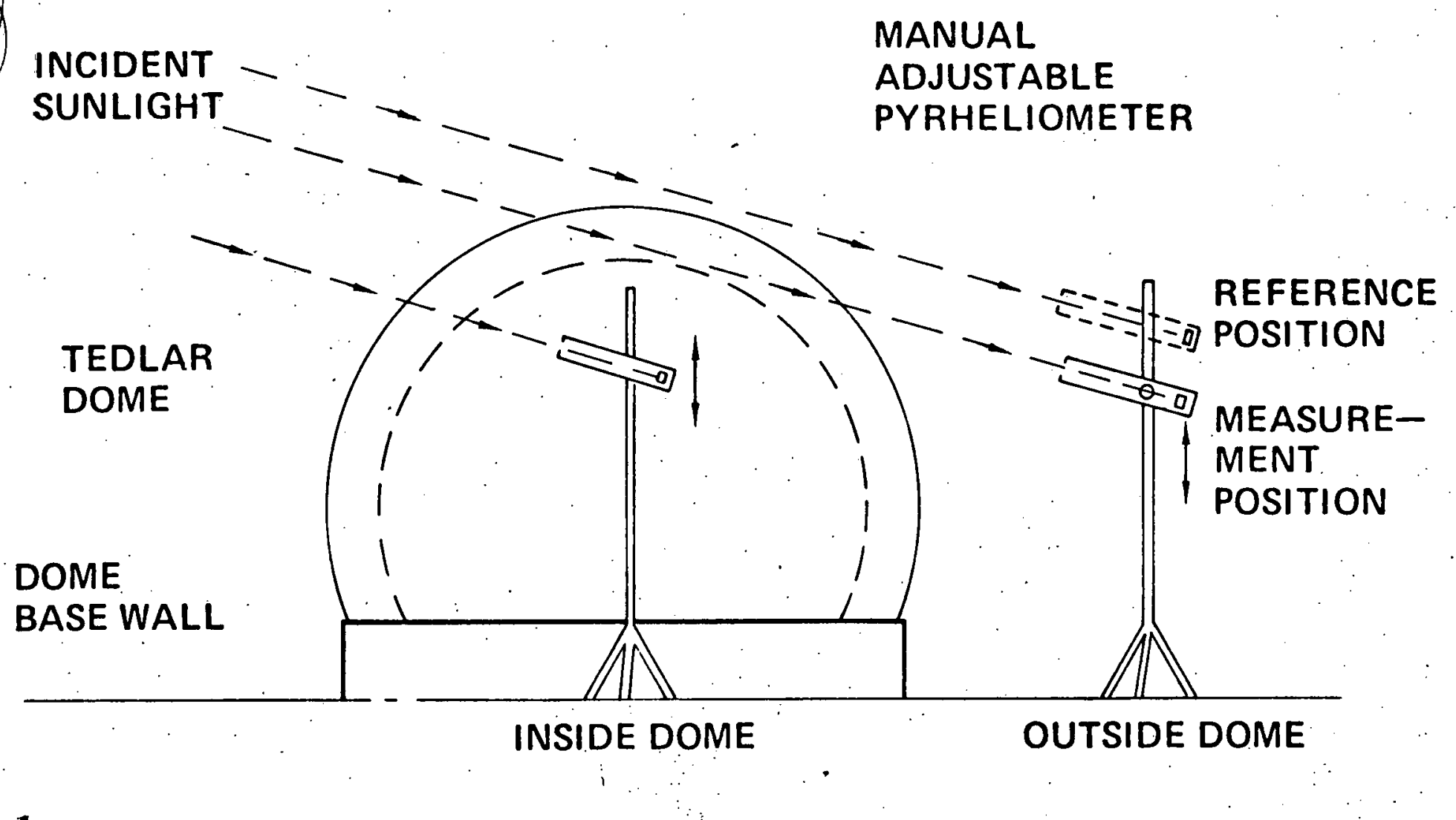




\section{Heliostat Reflectance Test}

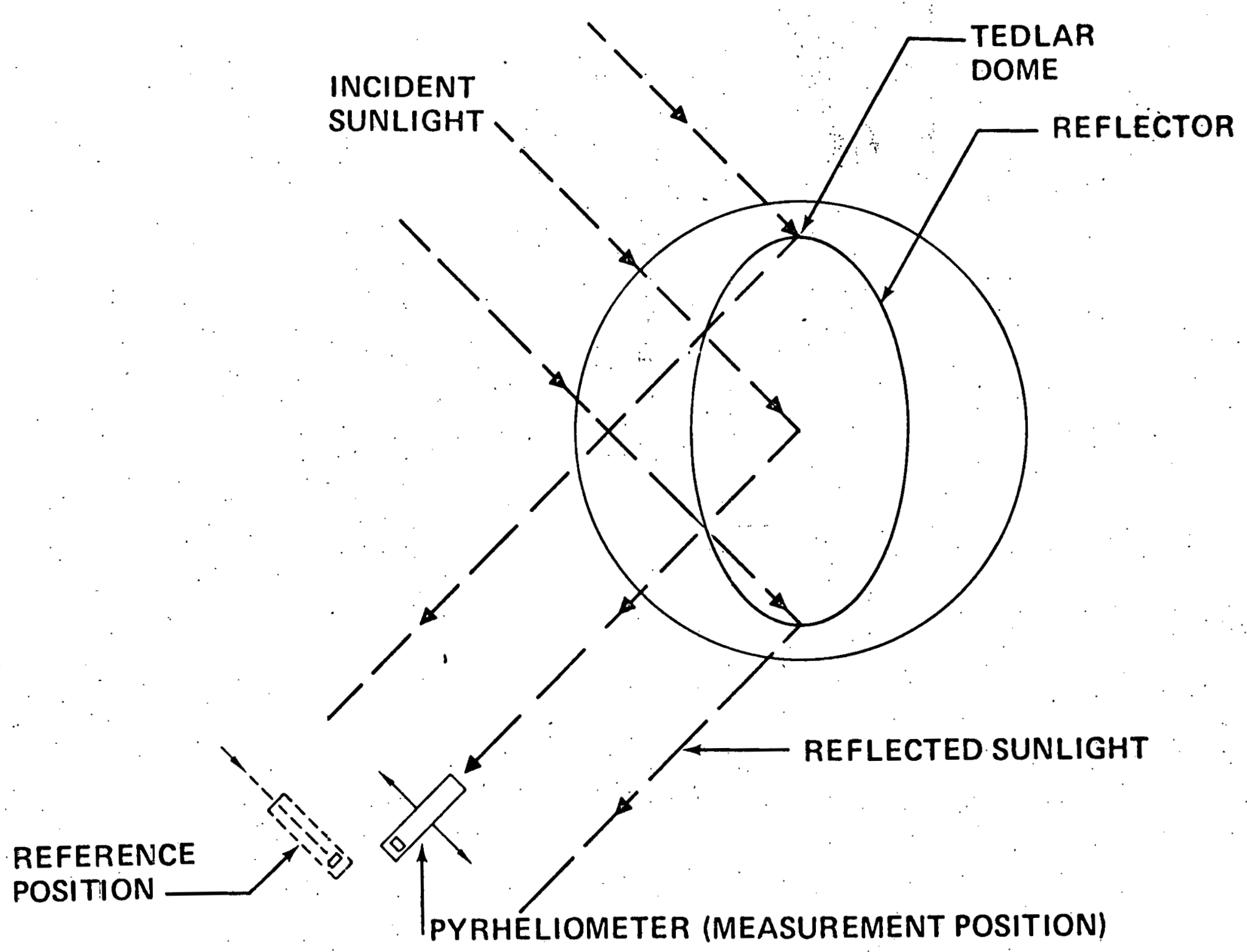




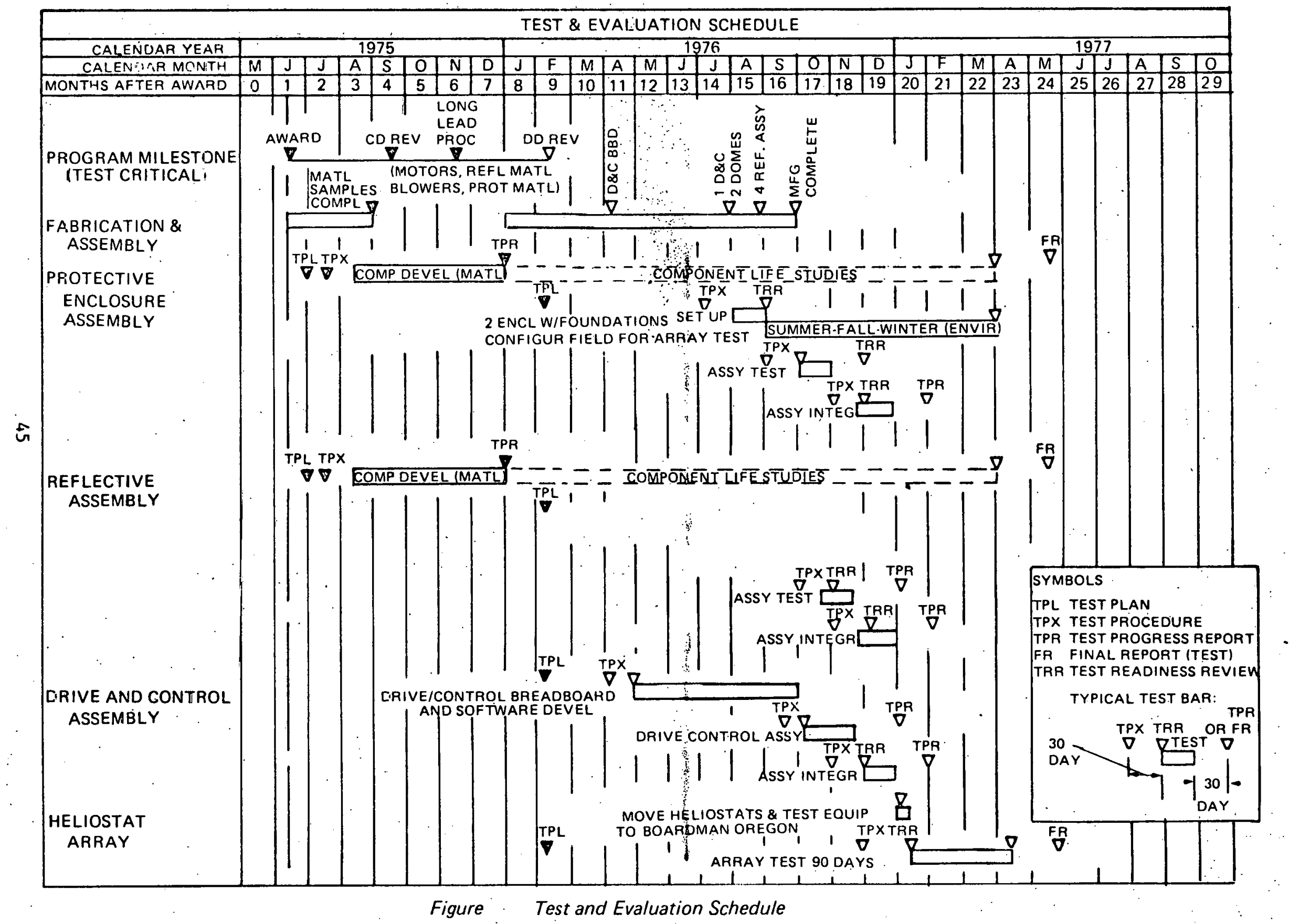




\begin{tabular}{|c|c|c|c|c|}
\hline bentivy & \multicolumn{2}{|c|}{ Drecription } & $\begin{array}{l}\text { Actual } \\
\text { thicknos } \\
\text { (mm) }\end{array}$ & $\underset{x}{\text { Transmission }}$ \\
\hline 7825 & Sienderd Tedles & Dupont roll polinh & .099 & 81.1 \\
\hline 7825 & Sianderd Tedtar & & .099 & 81.2 \\
\hline $7826 A$. & No epon & & .093 & 87.9 \\
\hline 78268 & No epon (bess soticts) & & .091 & 86.3 \\
\hline 78268 & No epon (Lexs colds) & & .093 & 87.9 \\
\hline 7027 & Standero Toolex & & .097 & 85.3 \\
\hline 8536 & No epon (less soilid) & - & .097 & 90.1 \\
\hline B152.1 & Standard Teilar & $\begin{array}{l}\text { Dunmore roll polish } \\
\text { uPont mill roll No. } 7827 \text { ) }\end{array}$ & .099 & 86.4 \\
\hline 8152.1 & Standard Tediar & & .100 & 84.7 \\
\hline 8152.2 & Standard Tacilar & & .099 & .84 .8 \\
\hline 8152.2 & Standard Tediar & & .099 & , 84.8 \\
\hline 8152.2 & Standard Todtat & . & .099 & B5.9 \\
\hline 8152.3 & Standard Tediar (unpolisiod) & (Virgin No. 7827) & .093 & 39.2 \\
\hline 8377 & Standaro Teciar & Dufont press polish & .107 & 78.3 \\
\hline$\varepsilon 378$ & (UV sureen and to epon) & & .093 & 87.5 \\
\hline
\end{tabular}

(50 pt calc., 0.5 cone angle and $12^{\circ}$ incident ensile)

Table 3.2-5: Specular Transmittance Data - Tedlar

\begin{tabular}{|c|c|c|c|}
\hline Sampla description & $\begin{array}{c}\text { Nominal } \\
\text { thickness } \\
\text { rim }\end{array}$ & $\begin{array}{c}\text { Actual } \\
\text { phichness } \\
\text { mi: }\end{array}$ & $\begin{array}{c}\text { Transmission } \\
\times\end{array}$ \\
\hline $\begin{array}{ll}\text { Aclar 22C las reccivudl } \\
\text { Aclar 22C } 10 \mathrm{~min}, 350.375^{\circ} \mathrm{F} .200 \mathrm{psi}\end{array}$ & .13 & $\begin{array}{l}.126 \\
.114\end{array}$ & $\begin{array}{l}63.3 \\
89.4\end{array}$ \\
\hline 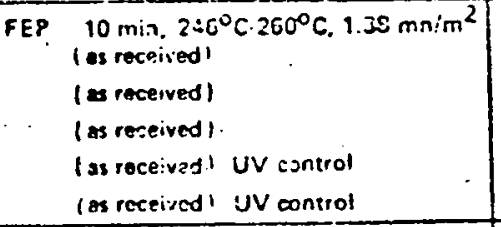 & $\begin{array}{l}.51 \\
.51 \\
.25 \\
.13 \\
.25 \\
.25\end{array}$ & $\begin{array}{l}.513 \\
.513 \\
.249 \\
.124 \\
.257 \\
.254\end{array}$ & $\begin{array}{l}80.3 \\
79.1 \\
86.3 \\
90.6 \\
86.2 \\
88.1\end{array}$ \\
\hline $\begin{array}{l}\text { Halar (Js receibcd) } \\
\left(20 \mathrm{~min}, 240^{\circ} \mathrm{C}, 1.33 \mathrm{mn} / \mathrm{m}^{2} \text {. slow cocl) }\right. \\
\left(5 \mathrm{~min} .240^{\circ} \mathrm{C}, 1.38 \mathrm{mn} / \mathrm{m}^{2} \text {. cuick cool) }\right. \\
\left(10 \mathrm{~min}, 240^{\circ} \mathrm{C} \& 1.39 \mathrm{mn} / \mathrm{m}^{2}\right) \\
\text { CTFe rich }\left(5 \mathrm{~min}, 222^{\circ} \mathrm{C}, 0.34 \mathrm{mn} / \mathrm{m}^{2}\right) \\
\text { No anioxident } \\
\text { Standard } \\
\text { Standard } \\
\text { Standard } \\
\left(8 \text { min: } 24 \mathrm{C}^{\circ} \mathrm{C}, 0.69 \mathrm{mn} / \mathrm{m}^{2}\right)\end{array}$ & $\begin{array}{l}.19 \\
.19 \\
.19 \\
.25 \\
- \\
- \\
.05 \\
.19 \\
.25 \\
.25\end{array}$ & $\begin{array}{l}.203 \\
.132 \\
.152 \\
.119 \\
.132 \\
.348 \\
.051 \\
.206 \\
.259 \\
.191\end{array}$ & $\begin{array}{l}39.2 \\
72.9 \\
78.4 \\
73.0 \\
78.5 \\
66.8 \\
84.6 \\
72.7 \\
68.3 \\
60.6\end{array}$ \\
\hline $\begin{array}{l}\text { Korad A.CV (as recoived) } \\
\text { Korad A.CV (as received) }\end{array}$ & $\begin{array}{l}.05 \\
.08\end{array}$ & $\begin{array}{l}.046 \\
.079\end{array}$ & $\begin{array}{l}75.6 \\
64.6\end{array}$ \\
\hline $\begin{array}{r}\text { Tefzel } 1000 \text { AE (as received) } \\
500 \text { AE (os recoired) }\end{array}$ & .25 & $\begin{array}{l}.254 \\
.117\end{array}$ & $\begin{array}{l}77.0 \\
84.1\end{array}$ \\
\hline
\end{tabular}

(120 incident, $0.5 \mathrm{com} .50 \mathrm{pt})$

Table 3.2-6: Specular Transmittance Data - Other Firms 
Table 3.2-7: Reflectance Data

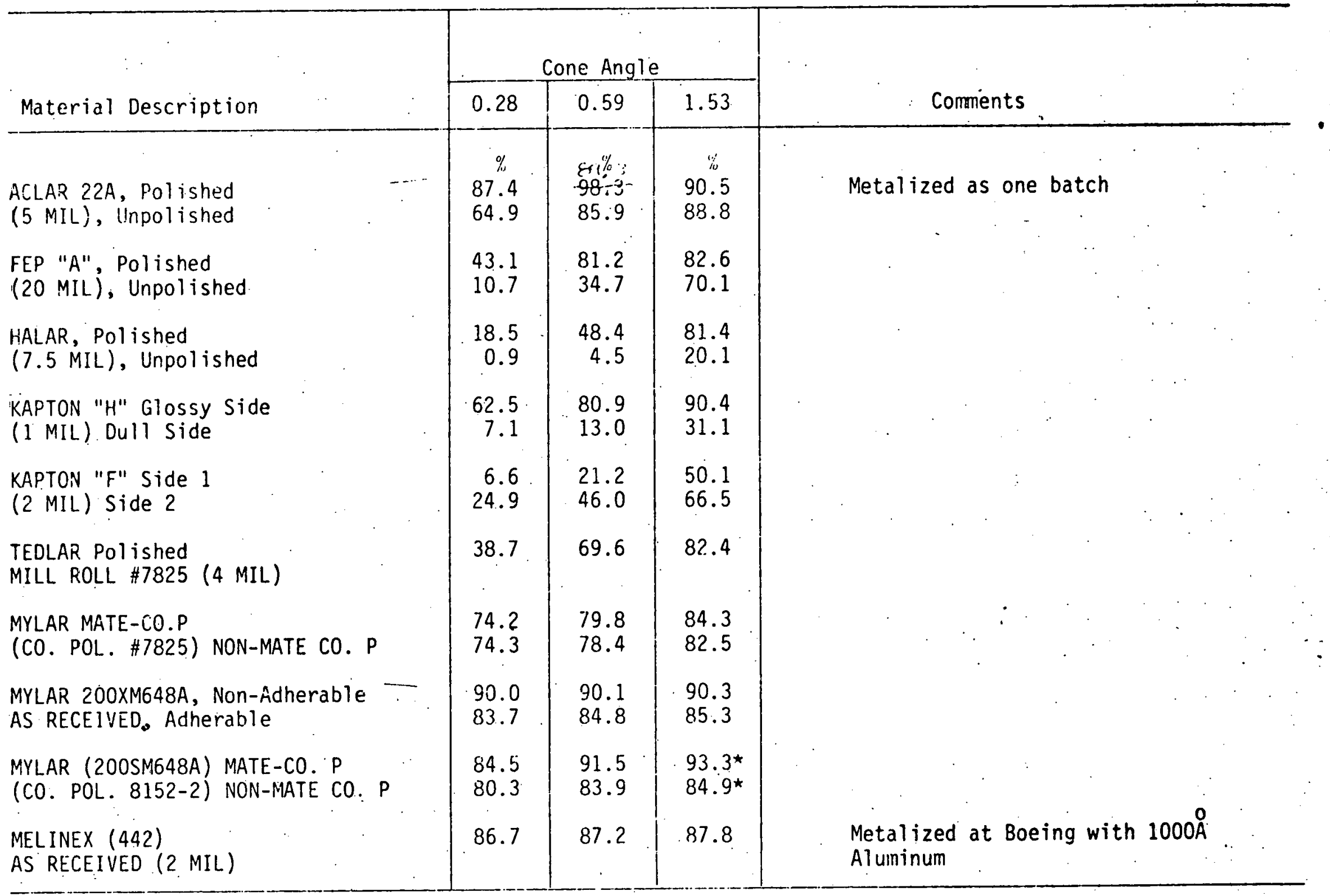

$\star$ Cone angle $1.43^{\circ}$ instead of $1.53^{\circ}$.

$\star \star$ Front surface vacuum-deposited, $12^{\circ}$ incident angle from normal 
Table 3.2-2: Tensile Proporties

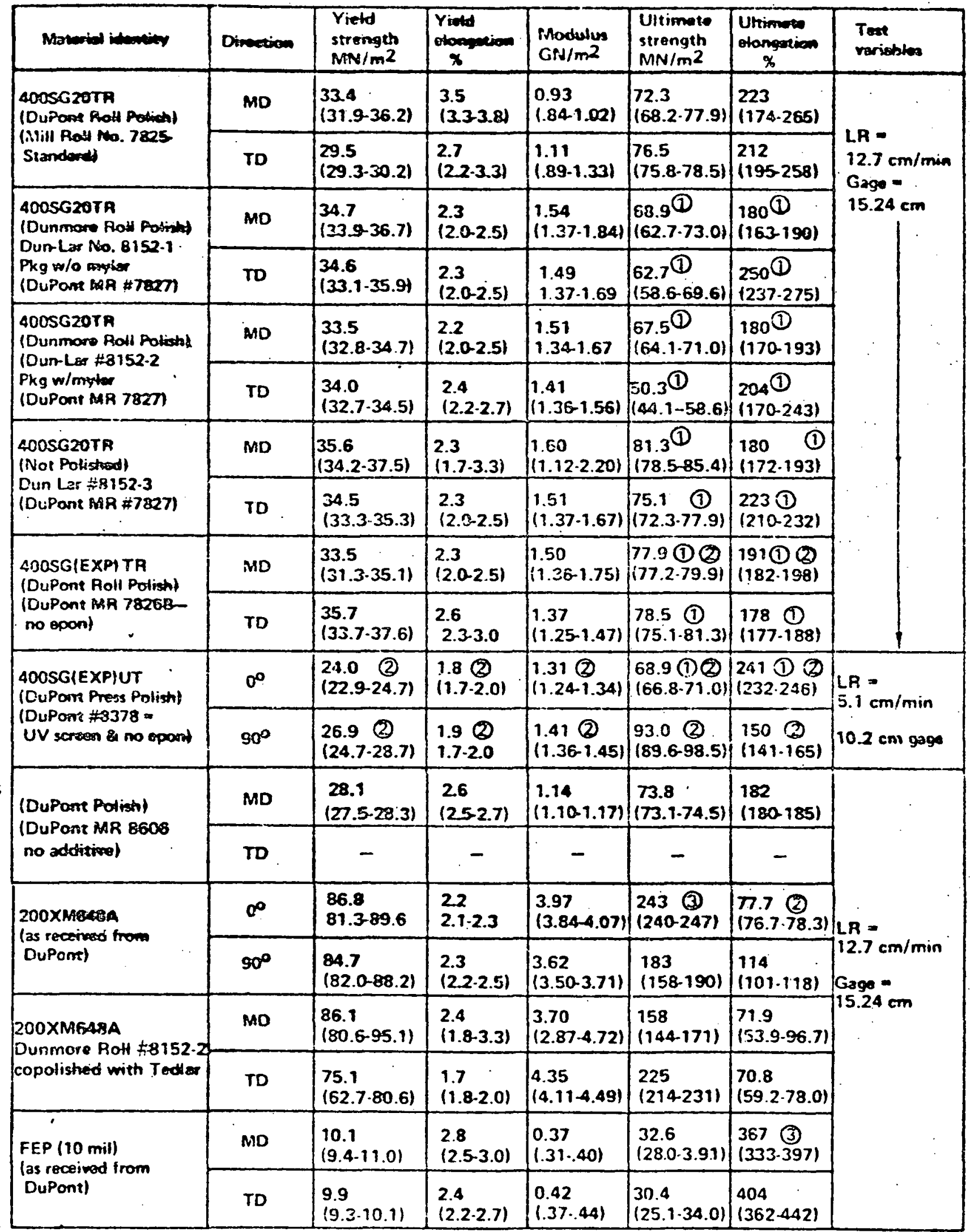

(1) Data may be conserverive dwe to significam momber of jow and nearjom tailures

(2) Avarnga of 3 spacimors

(3) Average of 4 


\section{Mechanical Properties versus Temperature}

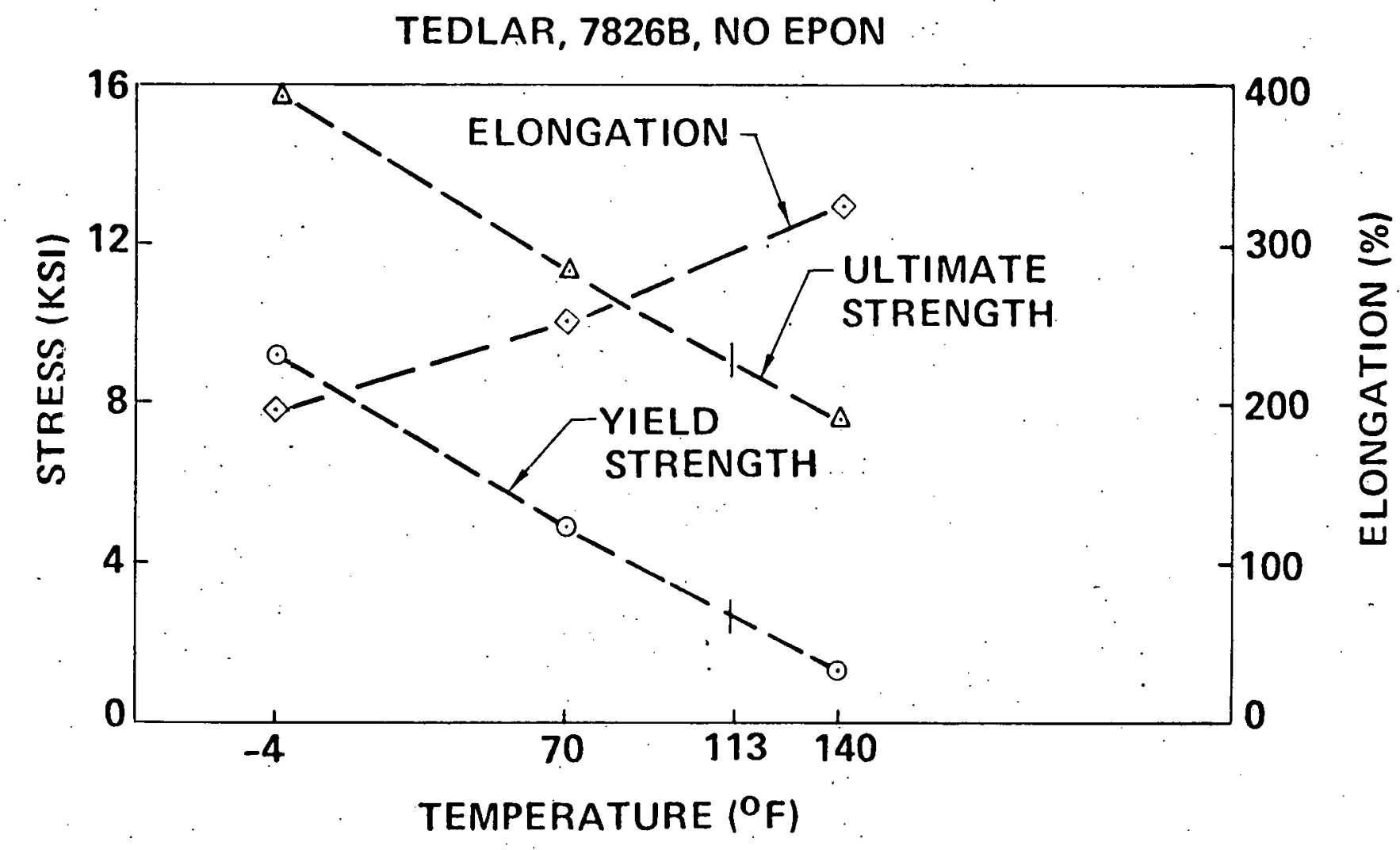




\section{Mechanical Properties versus Temperature}

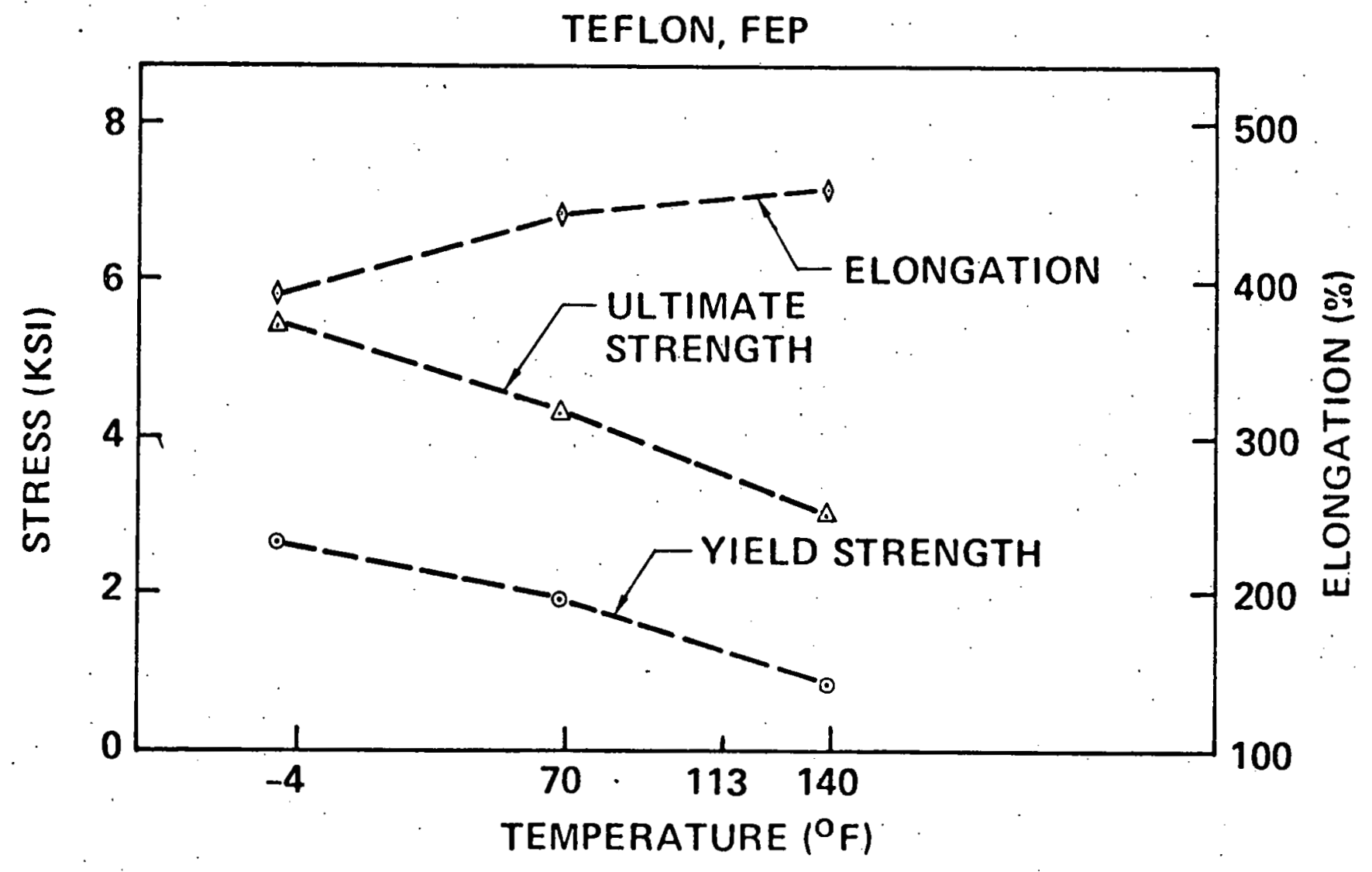




\section{Mechanical Properties versus Temperature}

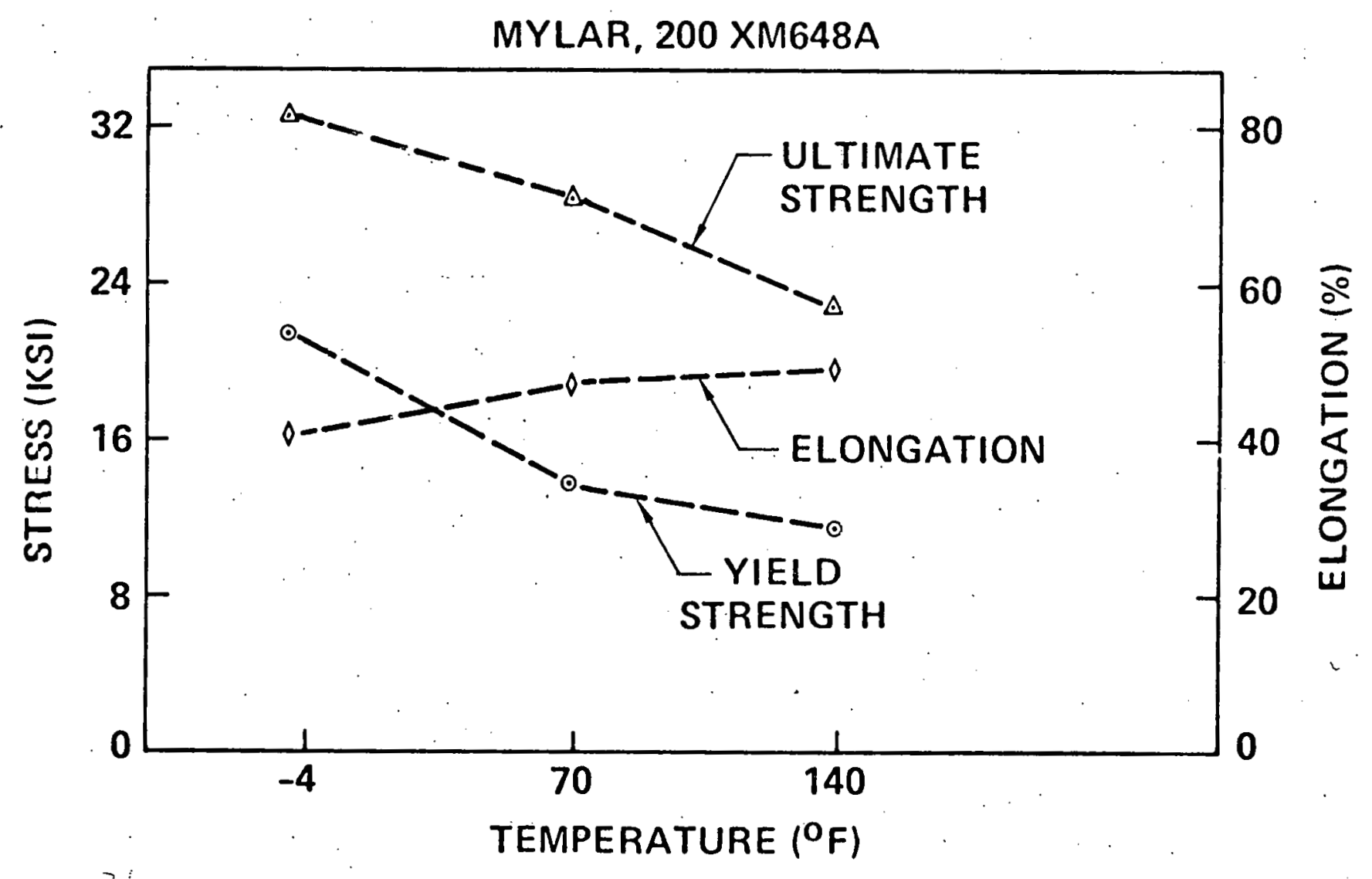


TABLE $6.2-2$

JOINT TENSILE TEST RESULTS

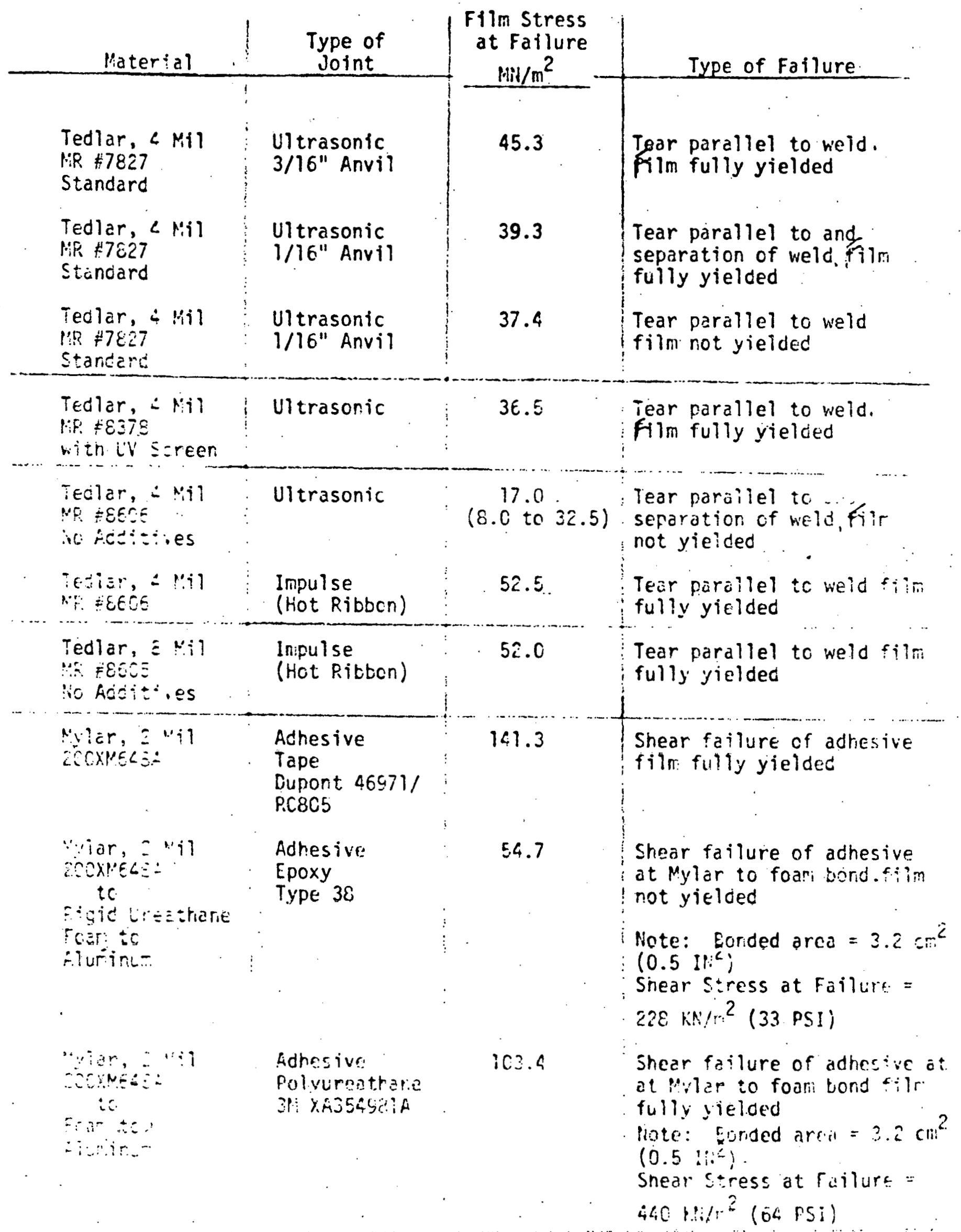




\section{Creep Tesi Setup}

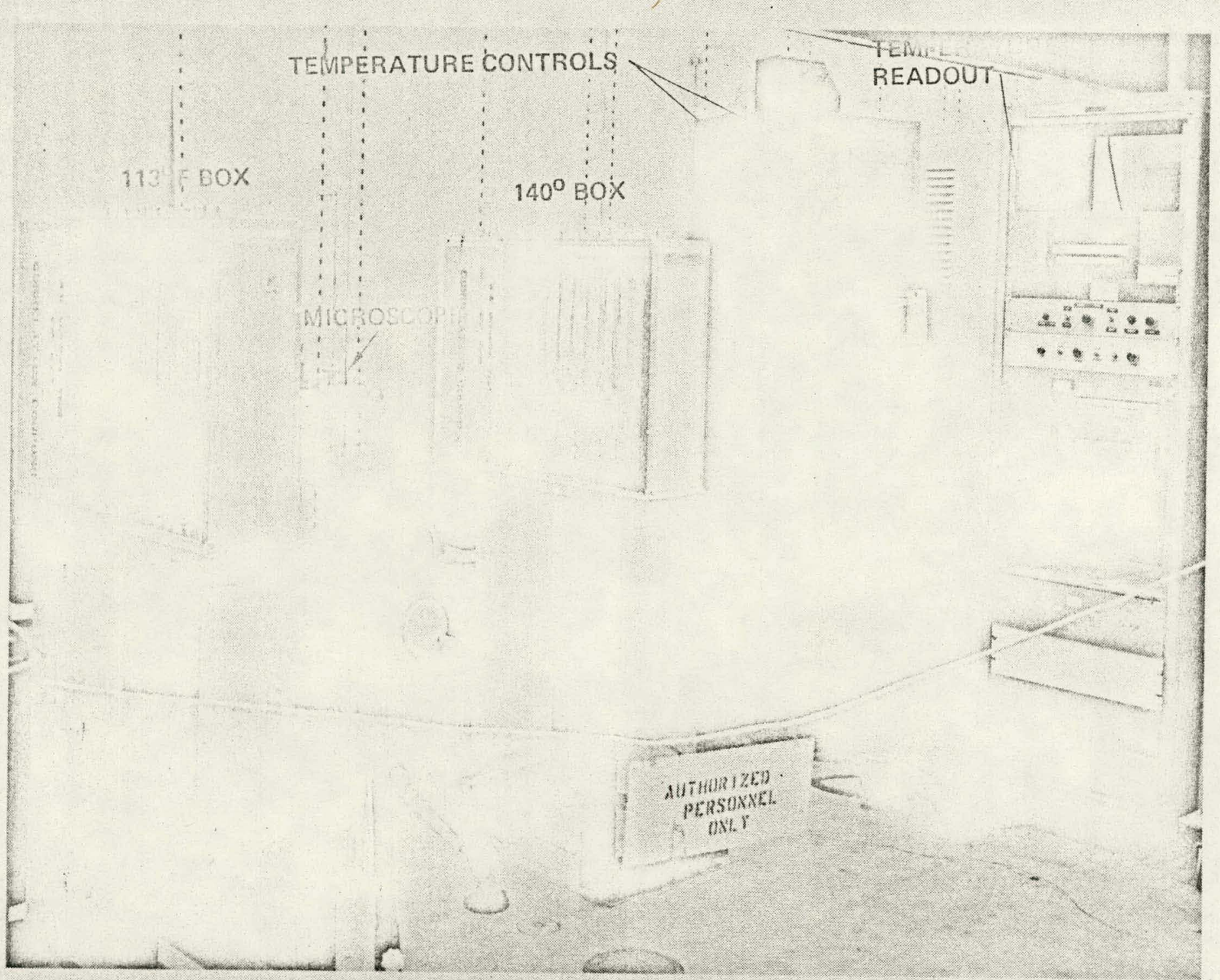


TABLE 3.2-4

CREEP TEST RESULTS

\begin{tabular}{|c|c|c|c|c|}
\hline \multirow[t]{2}{*}{ Material Identity } & $\begin{array}{l}\text { Stress } \\
\text { Level }\end{array}$ & $\begin{array}{l}\text { Test } \\
\text { Temp. }\end{array}$ & $\begin{array}{c}\text { Exposure } \\
\text { Time } \\
\end{array}$ & $\begin{array}{l}\text { Creep } \\
\text { Strain }\end{array}$ \\
\hline & $\mathrm{MN} / \mathrm{m}^{2}$ & ${ }^{\circ} \mathrm{C}$ & & \\
\hline $\begin{array}{l}\text { Tedlar, } 4 \mathrm{mil} \\
\text { MR 글 } \\
\text { No Epon, Less } \\
\text { Solids }\end{array}$ & 5.00 & 45 & $\begin{array}{r}50 \\
310 \\
890 \\
1660\end{array}$ & $\begin{array}{l}1.34 \\
1.53 \\
1.60 \\
1.61\end{array}$ \\
\hline \multirow[t]{2}{*}{$\begin{array}{l}\text { Tedlar, } 4 \text { mil } \\
\text { MR \#8378 } \\
\text { No Epon, } \\
\text { with UV Screen }\end{array}$} & 3.76 & 45 & $\begin{array}{r}45 \\
305 \\
885 \\
.1660\end{array}$ & $\begin{array}{l}1.06 \\
1.20 \\
1.34 \\
1.34\end{array}$ \\
\hline & 5.00 & 45 & $\begin{array}{r}45 \\
310 \\
890 \\
1660\end{array}$ & $\begin{array}{l}1.62 \\
1.83 \\
1.98 \\
1.99\end{array}$ \\
\hline $\begin{array}{l}\text { FEP, } 10 \mathrm{mil} \\
\text { As Received }\end{array}$ & 1.79 & 45 & $\begin{array}{r}50 \\
310 \\
890 \\
1660\end{array}$ & $\begin{array}{l}0.41 \\
0.44 \\
0.48 \\
0.48\end{array}$ \\
\hline \multirow[t]{3}{*}{$\begin{array}{l}\text { Mylar, } 2 \mathrm{mil} \\
200 \mathrm{MM} 648 \mathrm{~A} \\
\text { Virgin }\end{array}$} & 6.89 & 45 & $\begin{array}{r}45 \\
310 \\
885 \\
1660\end{array}$ & $\begin{array}{r}-0.02 \\
0.02 \\
0.02 \\
0.02\end{array}$ \\
\hline & 3.45 & 60 & $\begin{array}{r}40 \\
305 \\
885 \\
1660\end{array}$ & $\begin{array}{r}0.03 \\
-0.01 \\
-0.02 \\
-0.05\end{array}$ \\
\hline & 6.89 & 60 & $\begin{array}{r}45 \\
310 \\
890 \\
1660\end{array}$ & $\begin{array}{l}0.14 \\
0.14 \\
0.18 \\
0.15\end{array}$ \\
\hline
\end{tabular}


CREEP TEET ETSULTS

OF SEAMYJUINTS

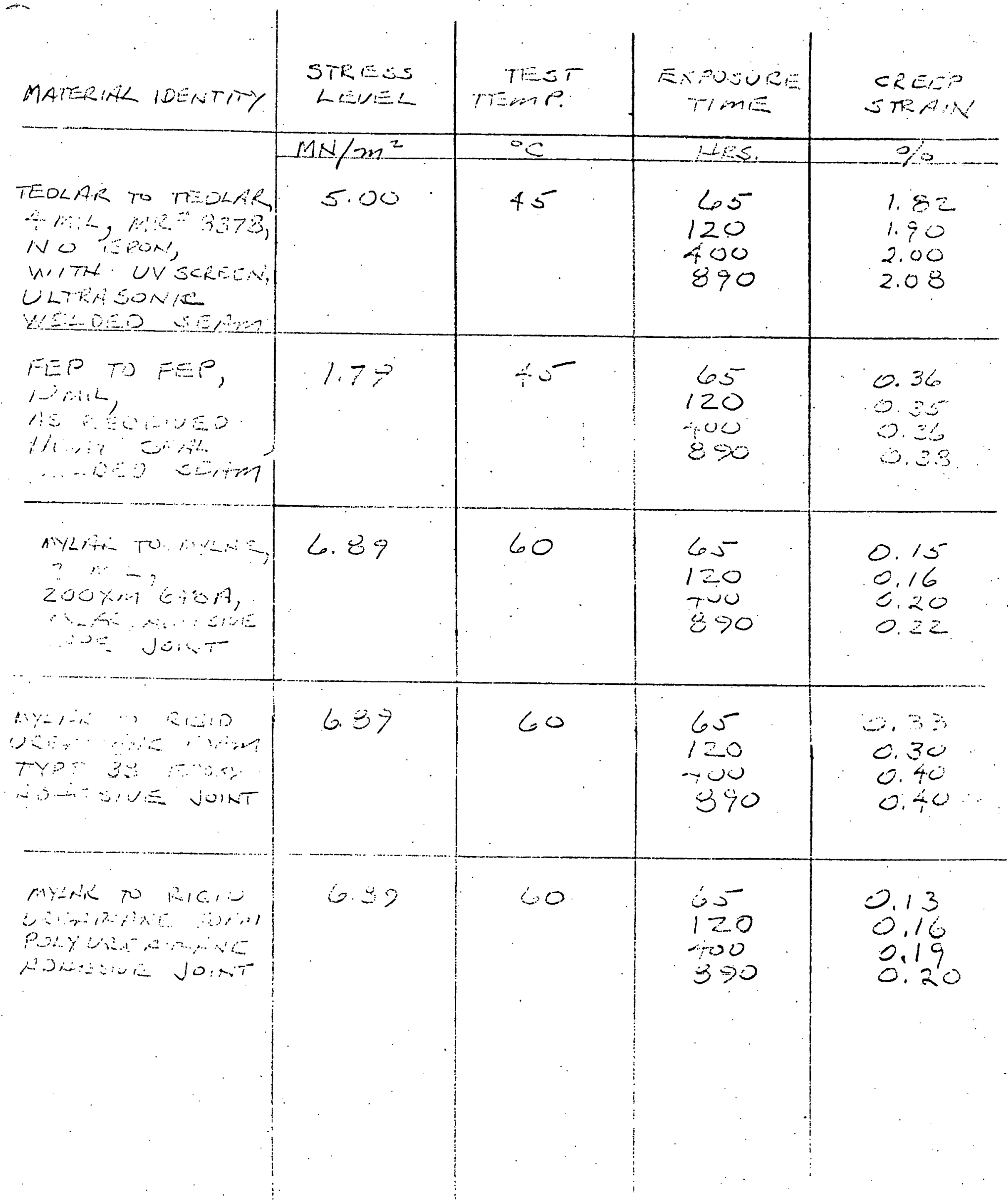




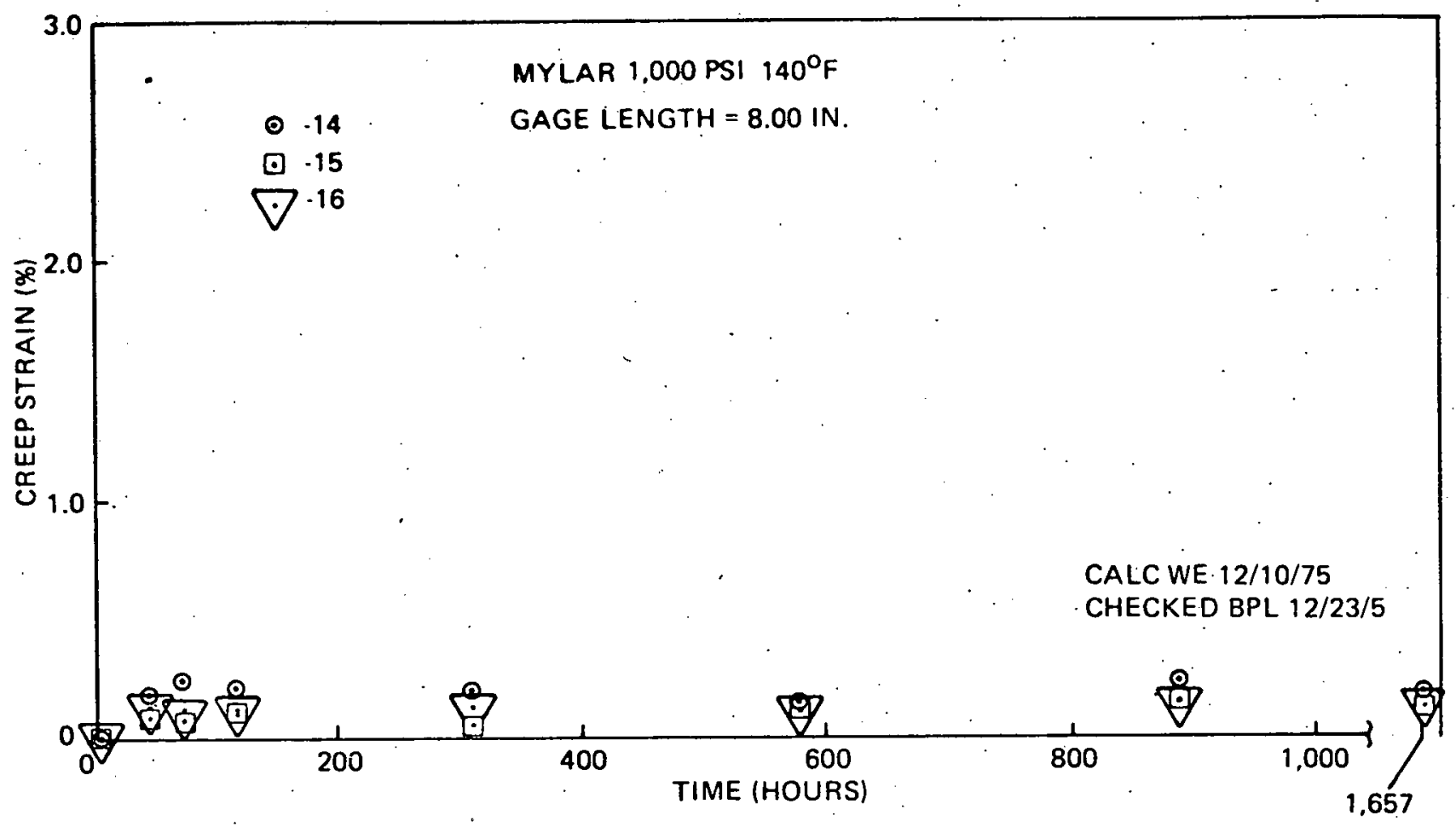

Figure . Time-Creep History-Mylar

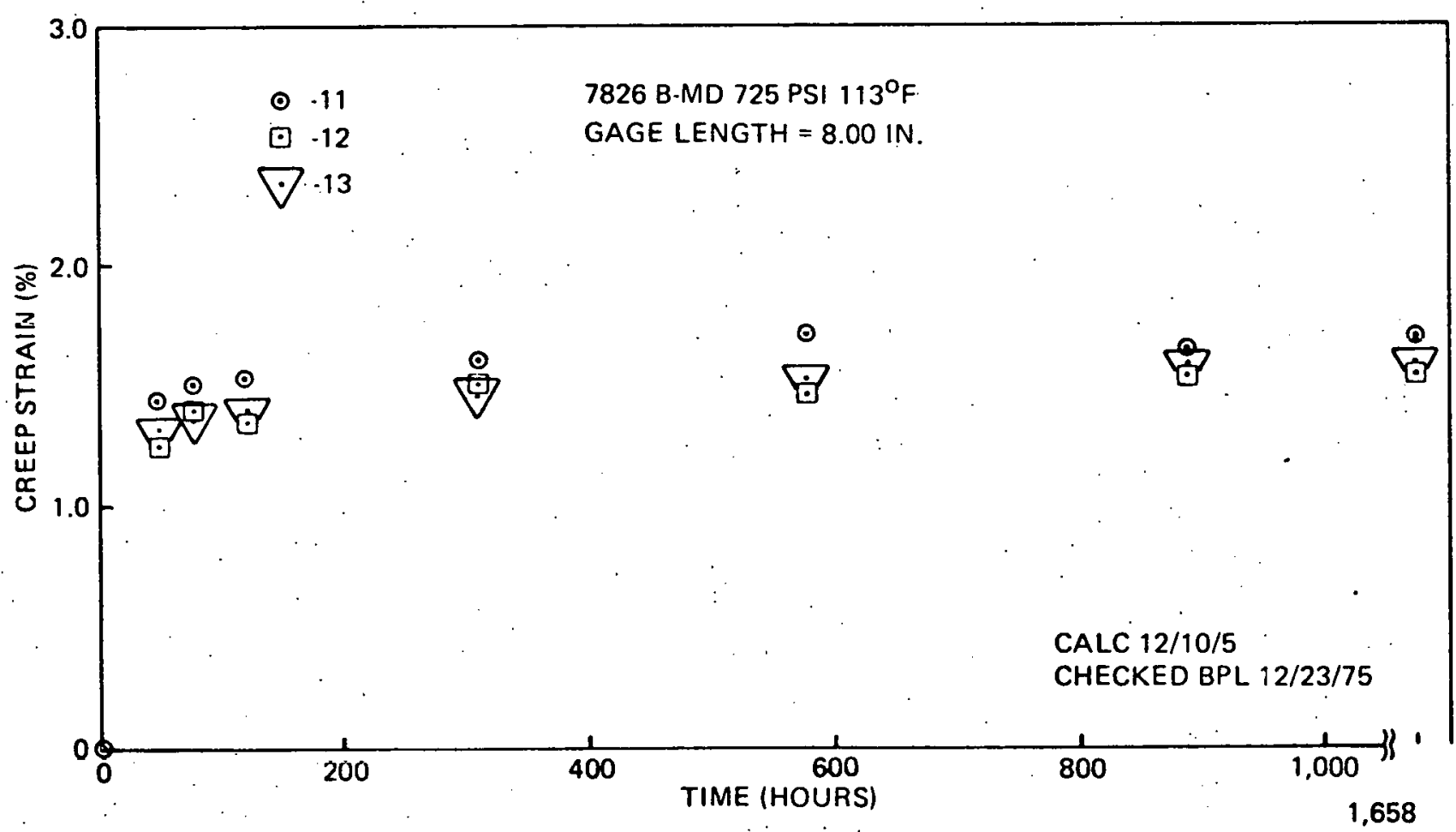

Figure . Time-Creep History-Tedlar 


\section{Accelerated UV Screening Specimens}

\begin{tabular}{|c|c|}
\hline Material Identity & Tests Performed \\
\hline $\begin{array}{l}\text { Tedlar, } 4 \text { Nill MR } 78268 \\
\text { fic hoditives. }\end{array}$ & $\begin{array}{l}\text { Microtensile, Transmittance Trosts } \\
\text { (Bascline Ecnie Material) }\end{array}$ \\
\hline $\begin{array}{l}\text { TeJlar, } 4 \text { Nil MR } \$ 7825 \\
\text { Staridard vith ErON }\end{array}$ & $\begin{array}{l}\text { Microtensile, Transrittance Tests } \\
\text { (Comitercial Grade Material) }\end{array}$ \\
\hline $\begin{array}{l}\text { Tediar, } 4 \text { Nit MR } \$ 8378 \\
\text { H/LV Sireen, lic EPOH }\end{array}$ & $\begin{array}{l}\text { Microterisile, Transfittance Tests } \\
\text { (U)truviclet Screer lieterial; }\end{array}$ \\
\hline $\begin{array}{l}\text { FEP Tefion, iC Mil } \\
\text { IS Received }\end{array}$ & $\begin{array}{l}\text { Micrcterisile, Frarsriiciance Tests } \\
\text { (Alternate Baseline Con:e Material) }\end{array}$ \\
\hline $\begin{array}{l}\text { Mylar } 2 \text { Mil (200xME48A) } \\
\text { Ailuirized (1000 Angstroms) } \\
\text { (list Surface Toward Source) }\end{array}$ & $\begin{array}{l}\text { Reflectaric: Tests } \\
\text { (Baseline Reflector Naterial) }\end{array}$ \\
\hline $\begin{array}{l}\text { Aluminized Nifrror } \\
\text { (Nicite) Sutstrate) }\end{array}$ & $\begin{array}{l}\text { Contaiination Control Specirien for } \\
\text { Reflectance }\end{array}$ \\
\hline Sapptitre Cisc & $\begin{array}{l}\text { Contanfriation Coritro: Specirien for } \\
\text { Transilitance }\end{array}$ \\
\hline
\end{tabular}


UV EXPOSURE TESTING

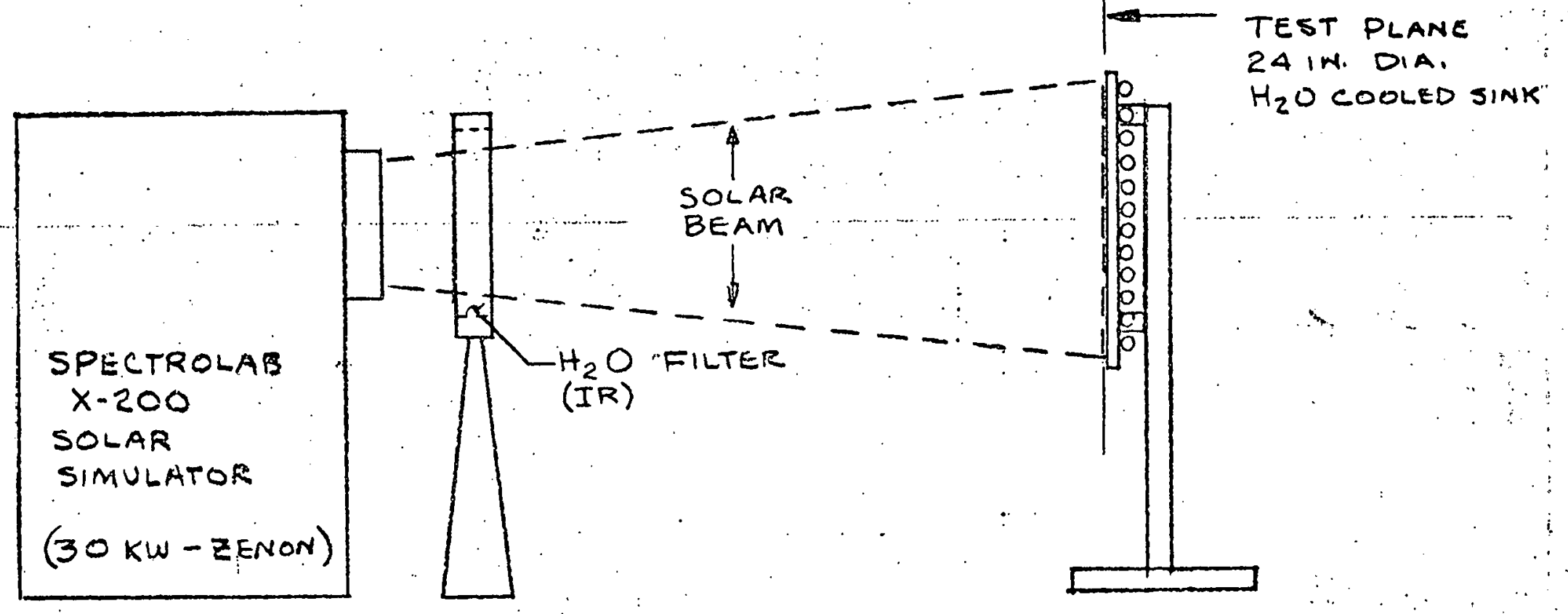

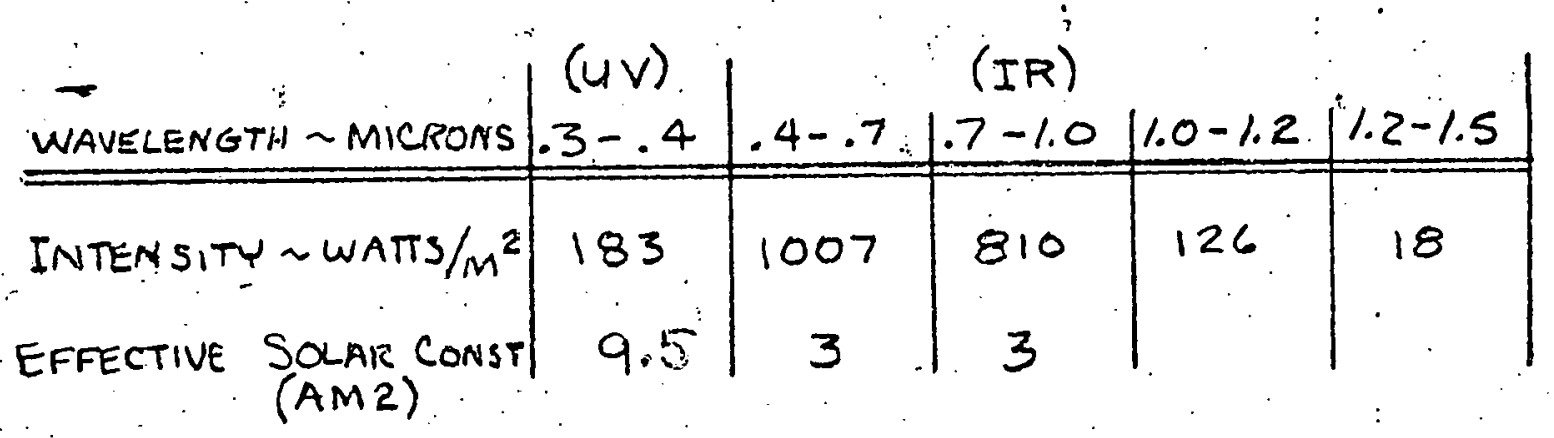

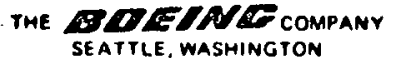


Specimen ${ }^{n /}$ ounting Board

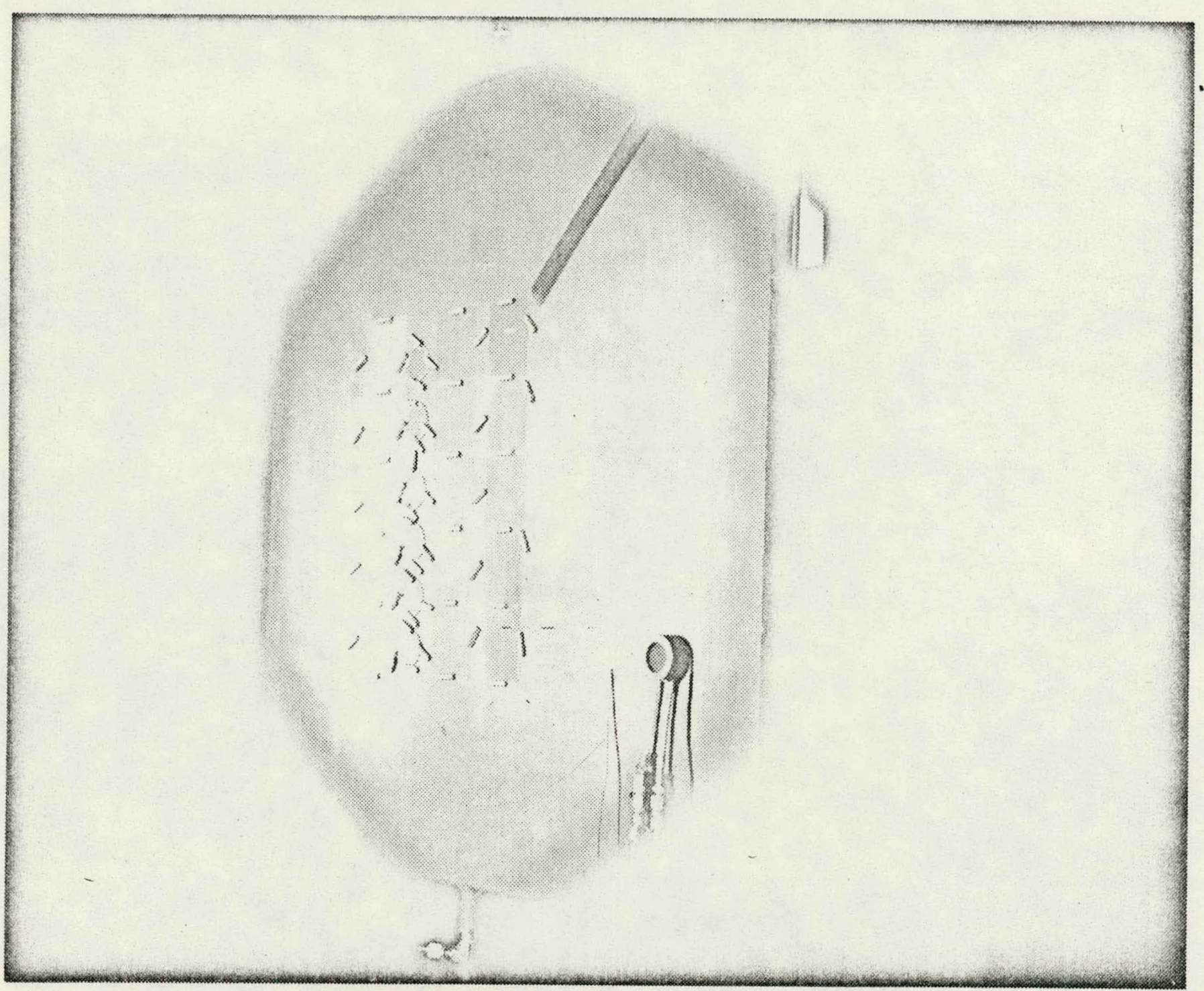

$-6$ 


\section{Accelerated Ultraviolet Degradation}

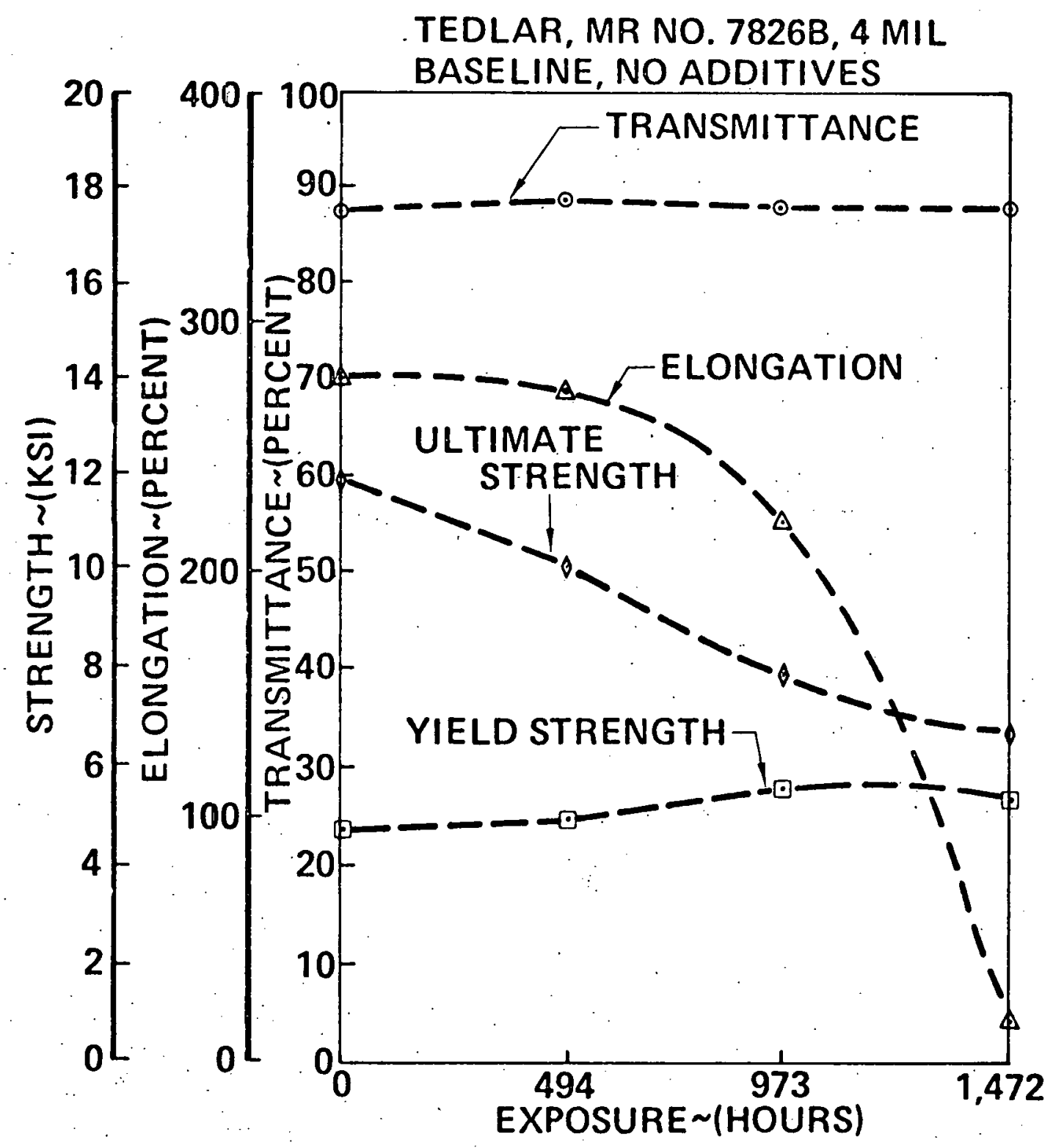




\section{Accelerated Ultraviolet Degradation}

TEDLAR, MR NO. 8378, $4 \mathrm{MIL}$

WITH UV SCREEN

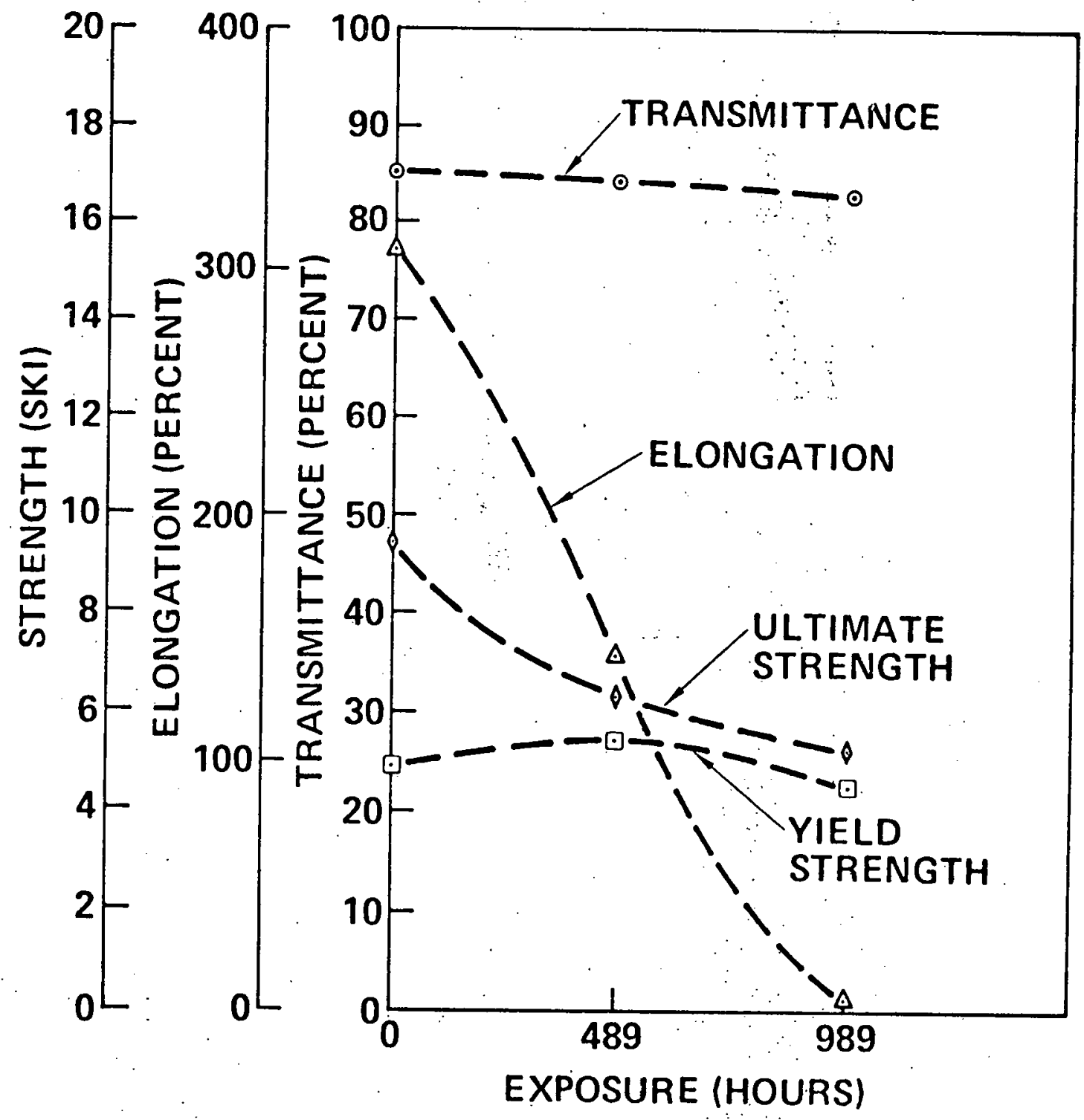




\section{Accelerated Ultraviolet Degradation}

TEDLAR' MR NO. $7825,4 \mathrm{MIL}$

STANDARD, WITH ADDITIVES

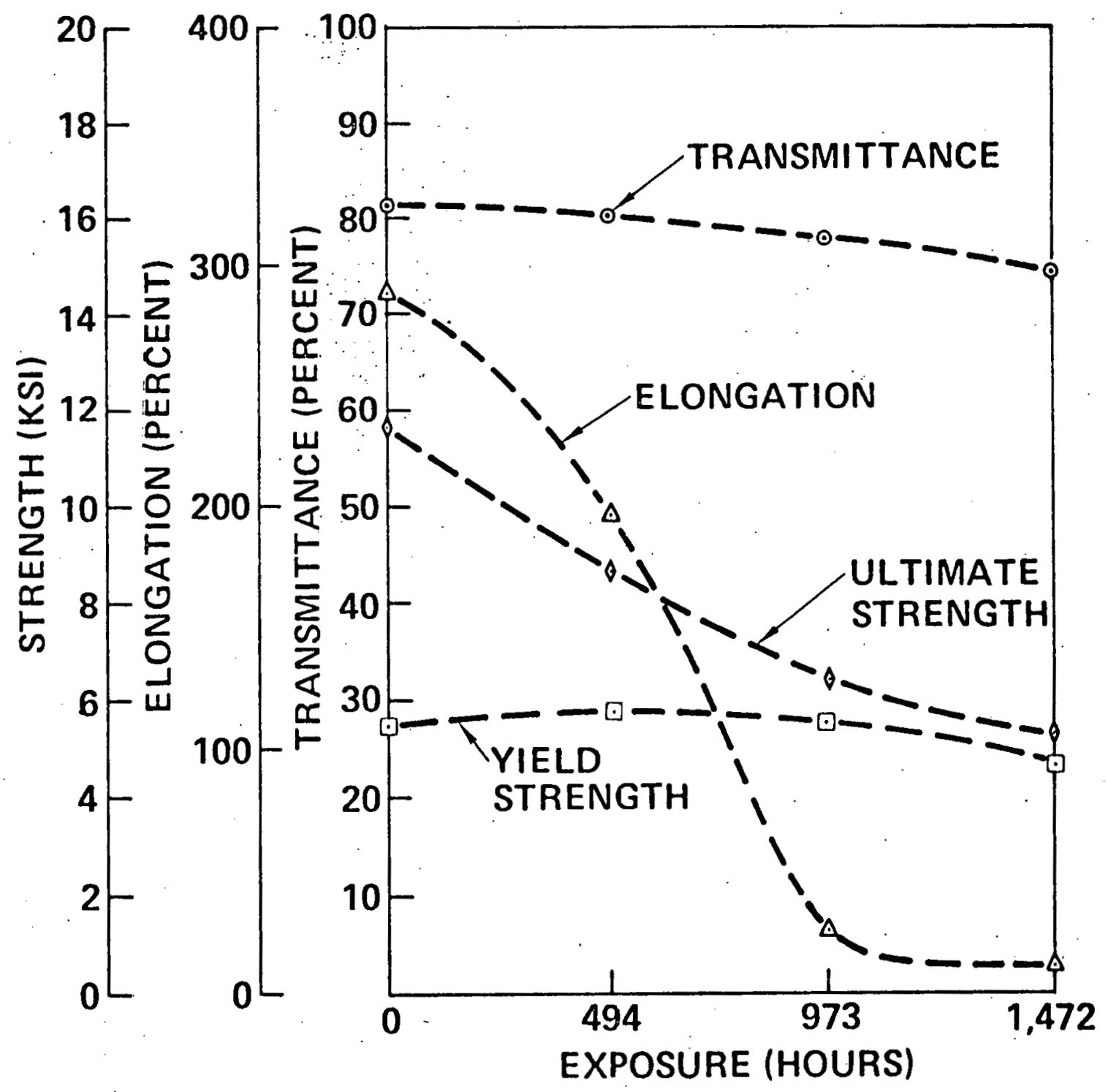




\section{Accelerated Ultraviolet Degradation}

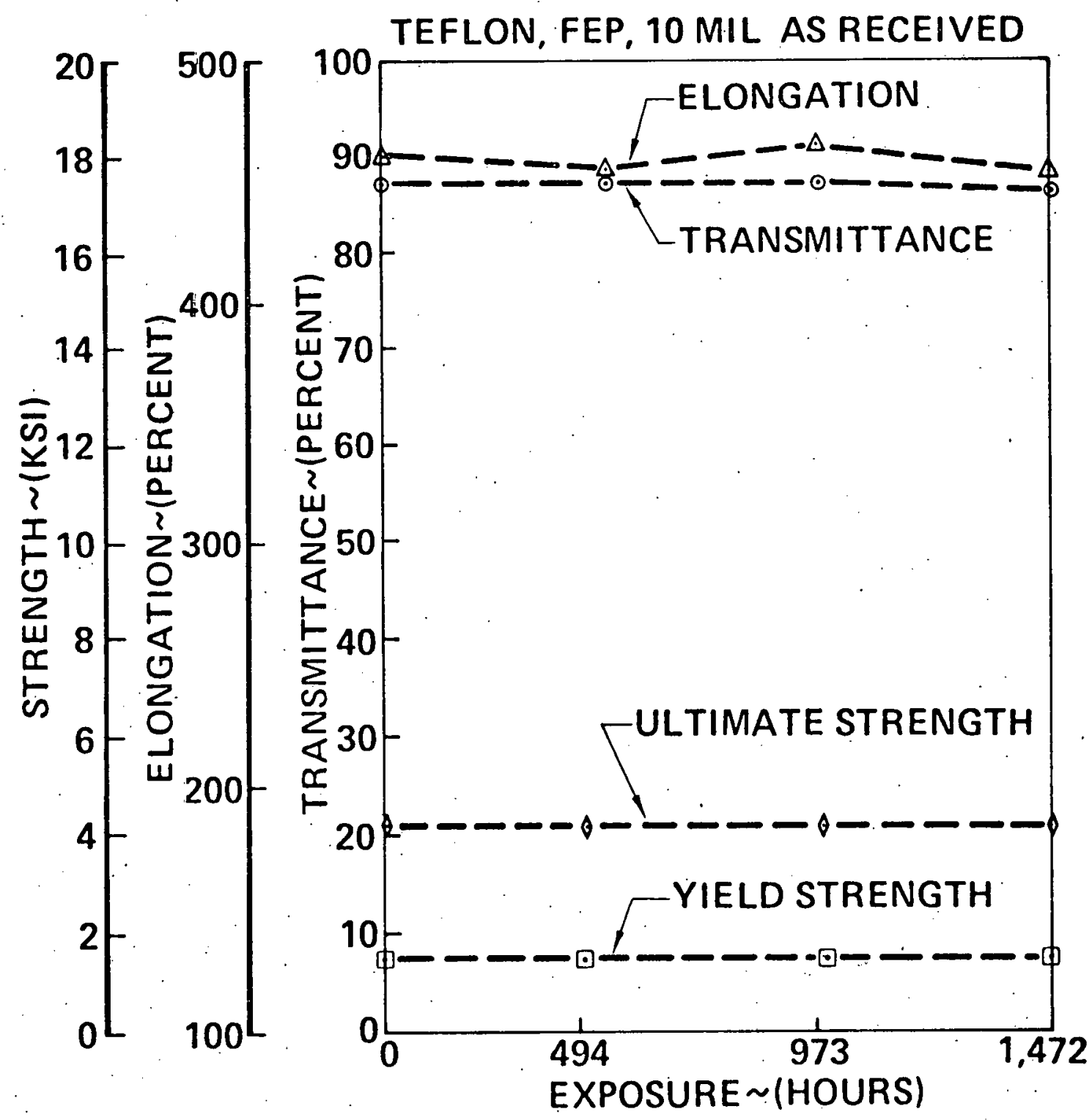




\section{Accelerated Ultraviolet Degradation}

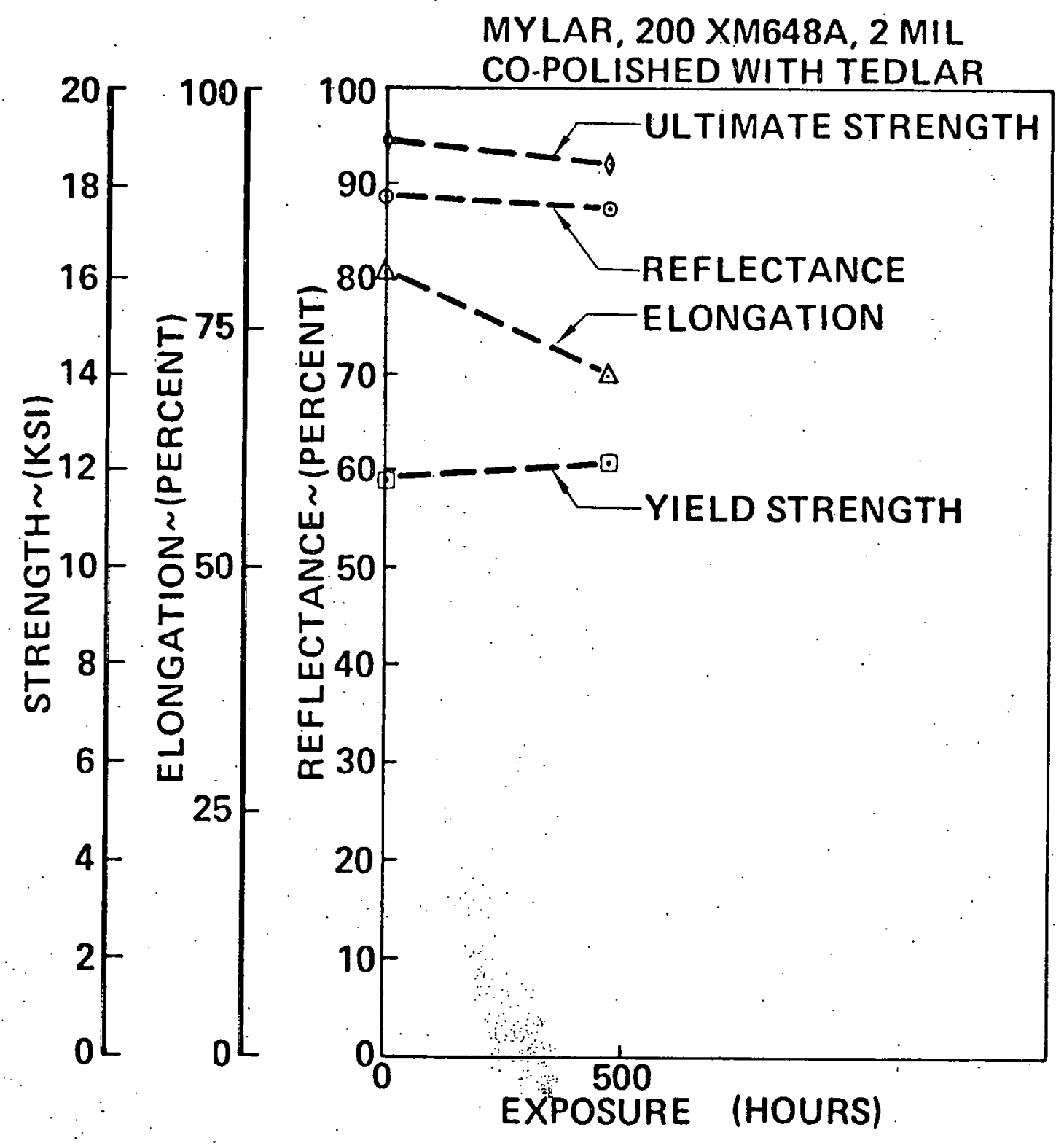




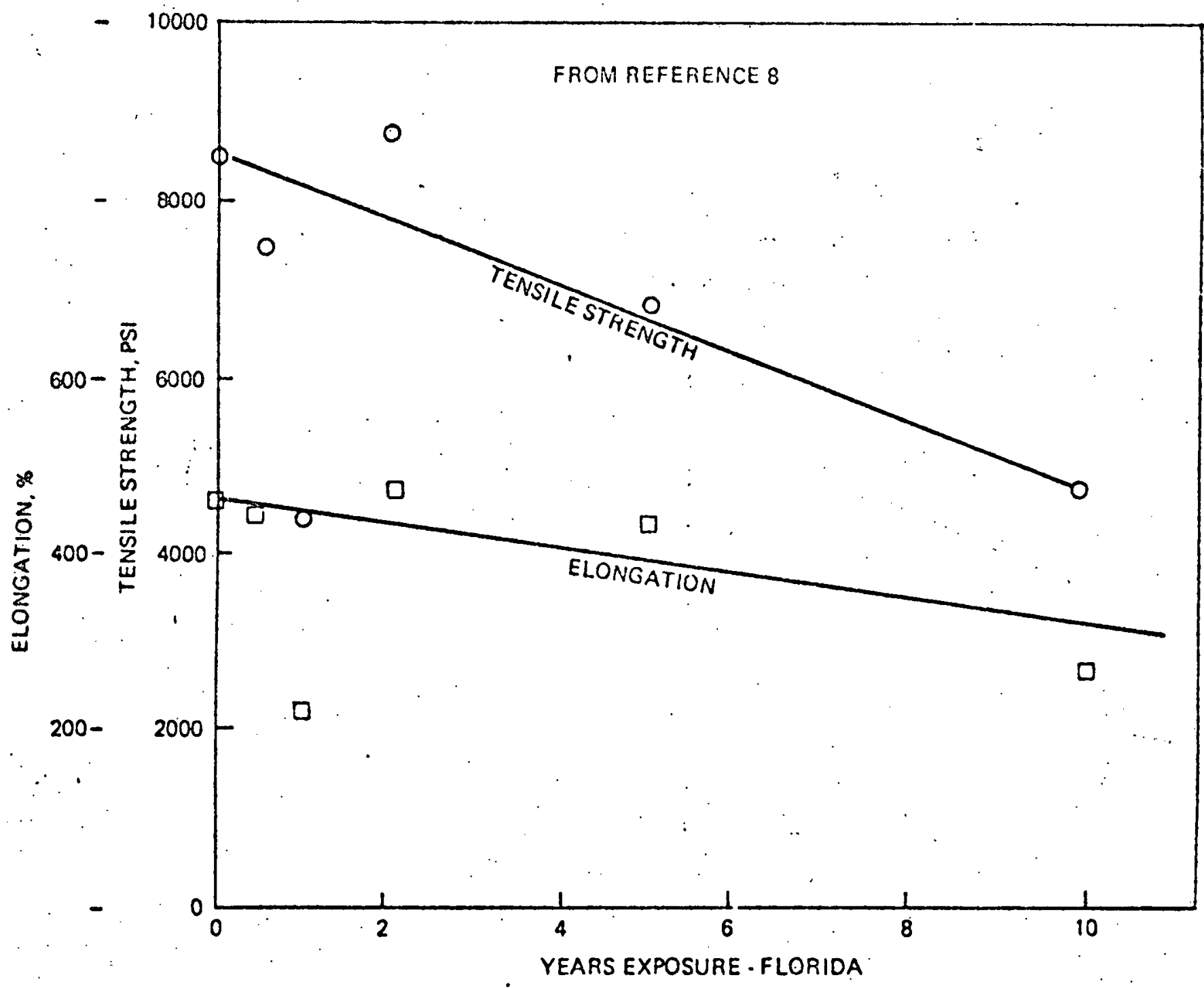

Figure 7.9. Strength vs. Outdoor Exposure for Transparent Tedlar 


\section{Desert Exposure Test Setup}

$\oplus$

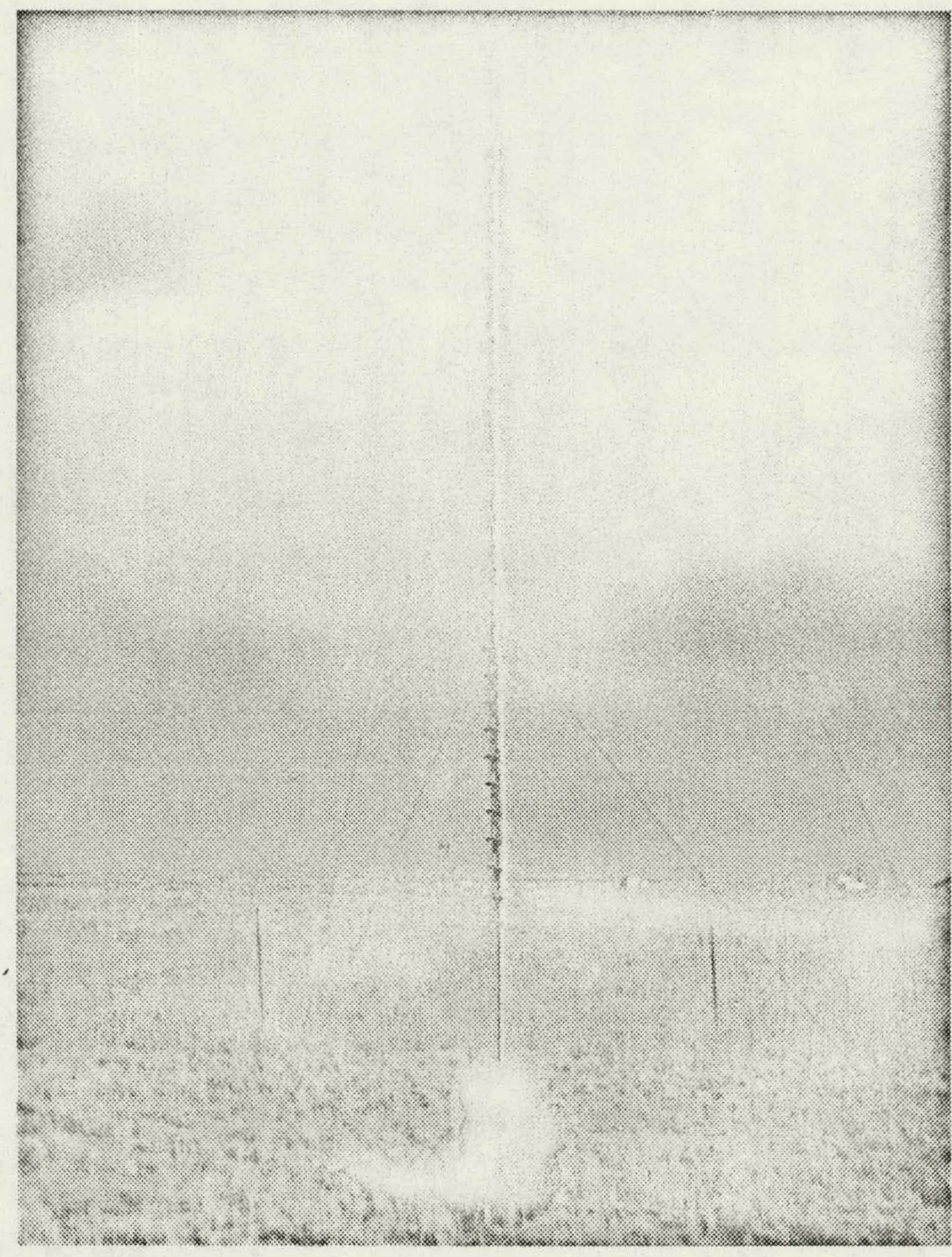


WEATHEROMETER HUMIDITY TESTS

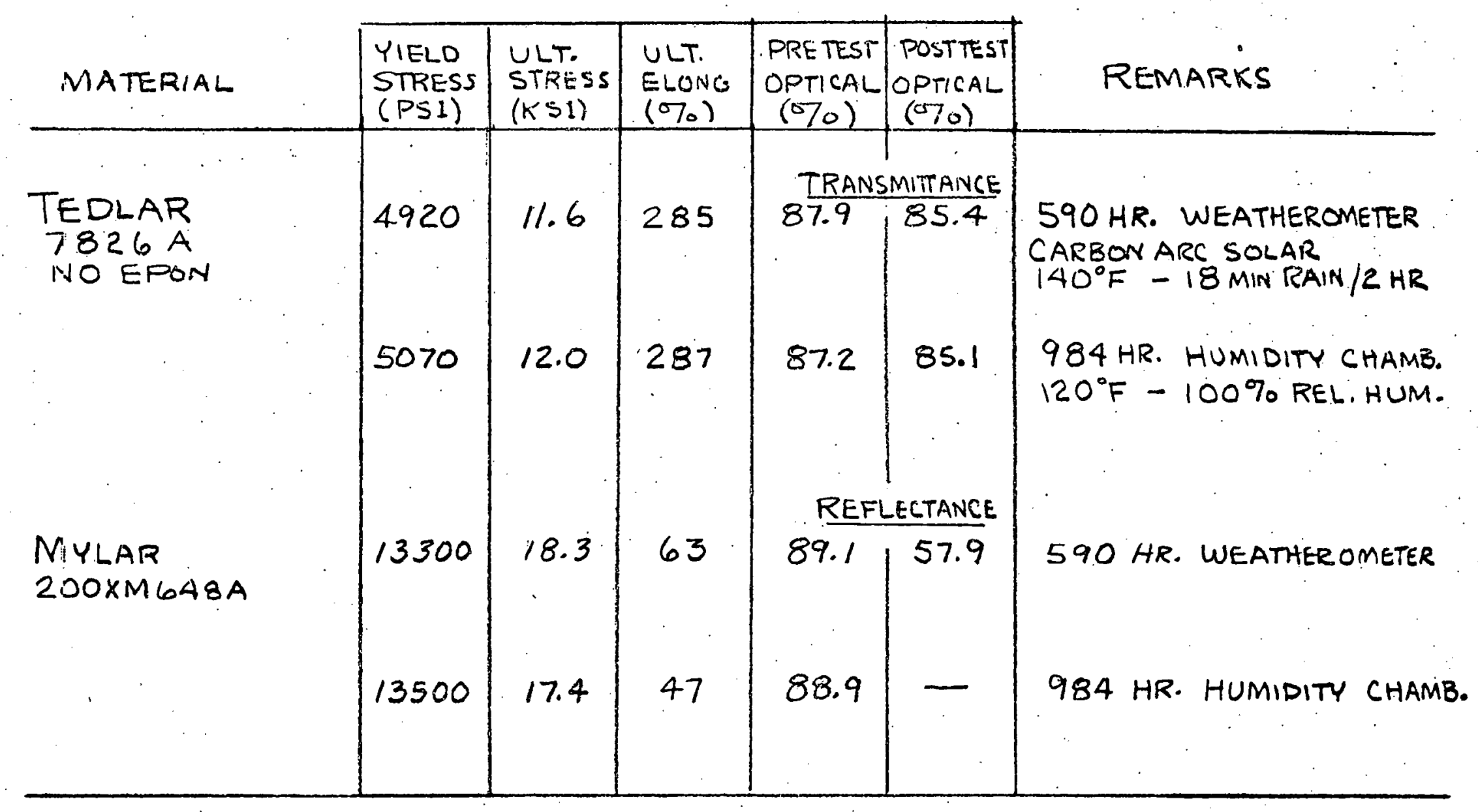


CORROSIVE EXPOSURES

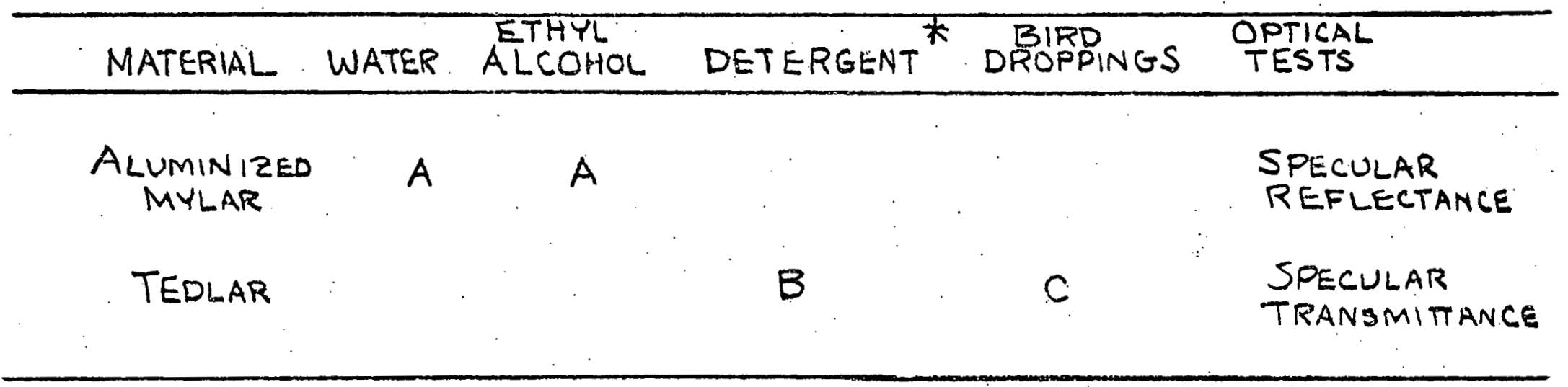

EXPOSURE DURATIONS

A - 40 WASHES ( 4 WASHES/ YR $\times 10$ YR. LIFE)

B - 20 HRS. ( BASED ON $1 / 2$ HR WASH $\times 4$ WASh/ YR $\times 10$ YR LIFE)

C - 30 DAYS

* Nl concentrate all purpose cleaner national labs - TOLEdo oH id 
- Exposures

1) Rain

2) Dust

3) Water/dust

4) Bird droppings

5) Bird droppings allowed to dry for 30 days

6) Detergent solution 23-1/2-hour soak
Cleaning method
A) Detergent/water
B) Detergent/water/soft brush
C) Compressed gas (GN2)
D) Vacuum cleaner
E) Alcohol (ethyl)
F). Distilled water.
G) Water rinse (tap)
H) Distilled water/soft brush

\begin{tabular}{|c|c|c|c|c|}
\hline \multirow[b]{2}{*}{ Material } & \multirow[b]{2}{*}{ Exposure } & \multirow{2}{*}{$\begin{array}{l}\text { Cleaning } \\
\text { method }\end{array}$} & \multicolumn{2}{|c|}{ Transmittance/reflectance } \\
\hline & & & Before exposure & After cleaning \\
\hline $\begin{array}{l}\text { Tedlar, 4-mil MR No. } 8378 \\
\text { with UV screen } \\
\qquad \\
\quad:\end{array}$ & $\begin{array}{l}\text { 1) } \\
\text { 2) } \\
\text { 2) } \\
\text { 2) } \\
\text { 2) } \\
\text { 3) } \\
\text { 3) } \\
\text { 3) } \\
\text { 3) } \\
\text { 3) } \\
\text { 4) }\end{array}$ & $\begin{array}{l}\text { N/A } \\
\text { A) } \\
\text { B) } \\
\text { C) } \\
\text { D) } \\
\text { G) } \\
\text { B) } \\
\text { C) } \\
\text { D) } \\
\text { G) } \\
\text { A) }\end{array}$ & $\begin{array}{l}\text { 84.6\% transmittance } \\
84.6 \% \text { transmittance } \\
84.6 \% \text { transmittance } \\
84.6 \% \text { transmitţance } \\
84.6 \% \text { transmittance } \\
84.6 \% \text { transmittance } \\
84.6 \% \text { transmittance } \\
84.6 \% \text { transmittance } \\
84.6 \% \text { transmittance } \\
84.6 \% \text { transmittance } \\
84.6 \% \text { transmittance } \\
84.6 \% \text { transmittance }\end{array}$ & $\begin{array}{l}\text { No change } \\
\text { No change } \\
\text { No change } \\
80.6 \% \\
73.0 \% \\
\text { No change } \\
\text { Unacceptable } \\
82.6 \% \\
81.0 \% \\
72.0 \% \\
\text { Unacceptable } \\
\text { Unacceptable }\end{array}$ \\
\hline . & $\begin{array}{l}\text { 4) } \\
\text { 5) } \\
6)\end{array}$ & $\begin{array}{l}\text { B) } \\
\text { B) } \\
\text { G) }\end{array}$ & $\begin{array}{l}84.6 \% \text { transmittance } \\
84.6 \% \text { transmittance } \\
84.6 \% \text { transmittance }\end{array}$ & $\begin{array}{l}84.0 \% \\
83.8 \% \\
84.0 \%\end{array}$ \\
\hline $\begin{array}{l}\text { Mylar, 2-mil aluminized } \\
200 \text { XM 648A } \\
\end{array}$ & $\begin{array}{l}\text { 1) } \\
\text { 1) } \\
\text { 2) } \\
\text { 2) } \\
\text { 2) } \\
\text { 3) } \\
\text { 3) } \\
\text { 3) }\end{array}$ & $\begin{array}{l}\text { E) } \\
\text { F) } \\
\text { C) } \\
\text { E) } \\
\text { F) } \\
\text { E) } \\
\text { F) } \\
\text { H) }\end{array}$ & $\begin{array}{l}90.0 \% \text { reflectance } \\
90.0 \% \text { reflectance } \\
90.0 \% \text { reflectance } \\
90.0 \% \text { reflectance } \\
90.0 \% \text { reflectance } \\
90.0 \% \text { reflectance } \\
90.0 \% \text { reflectance } \\
90.0 \% \text { reflectance }\end{array}$ & $\begin{array}{l}\text { No change } \\
\text { No change } \\
97.3 \% \\
89.4 \% \\
85.5 \% \\
\text { Unacceptable } \\
\text { Unacceptable } \\
88.5 \%\end{array}$ \\
\hline
\end{tabular}




\section{PLANS FOR NEXT REVIEW PERIOD}

- DELIVER FINAL - FIRST QUARTERLY REPORT

- INCORPORATE ERDA/SANDIA COMMENTS

- DELIVER DRAFT - 2ND QUARTERLY REPORT

- DELIVER DEETAILED DESIGN REVIEW REPORT

INCORPORATE ERDA/SANDIA COMMENTS

- PREPARE FOR PROJECT REVIEW - APRIL 20

आ

- COMPLETEEROCUREMENT ORDERS

$\therefore$

- COMPLETE DRAWING RELEASE

- INITIATE FABRICATION

- TOOLING

- RESEARCH EXPERIMENT HARDWARE 


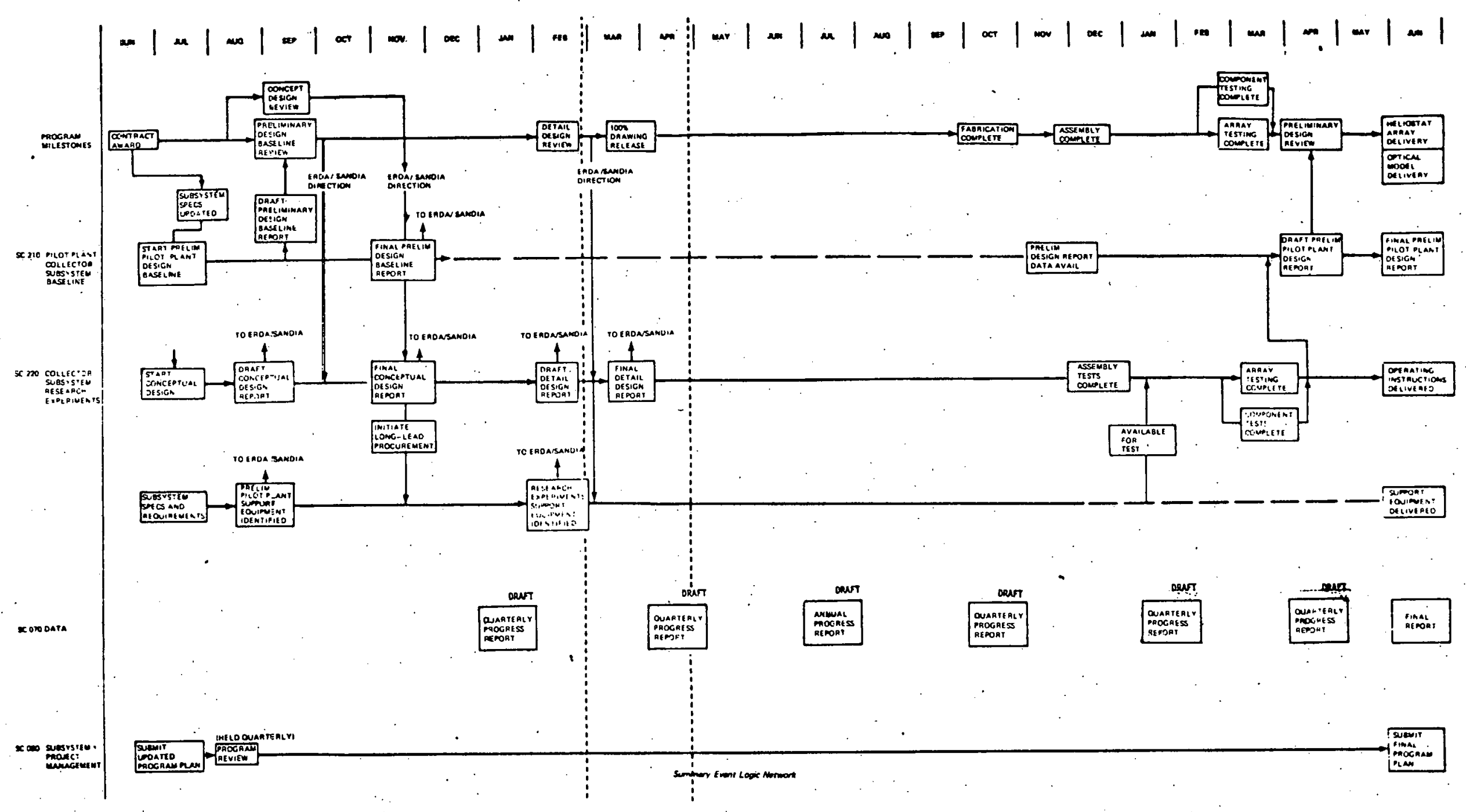


RESULTS

- 4900 hELIOSTATS
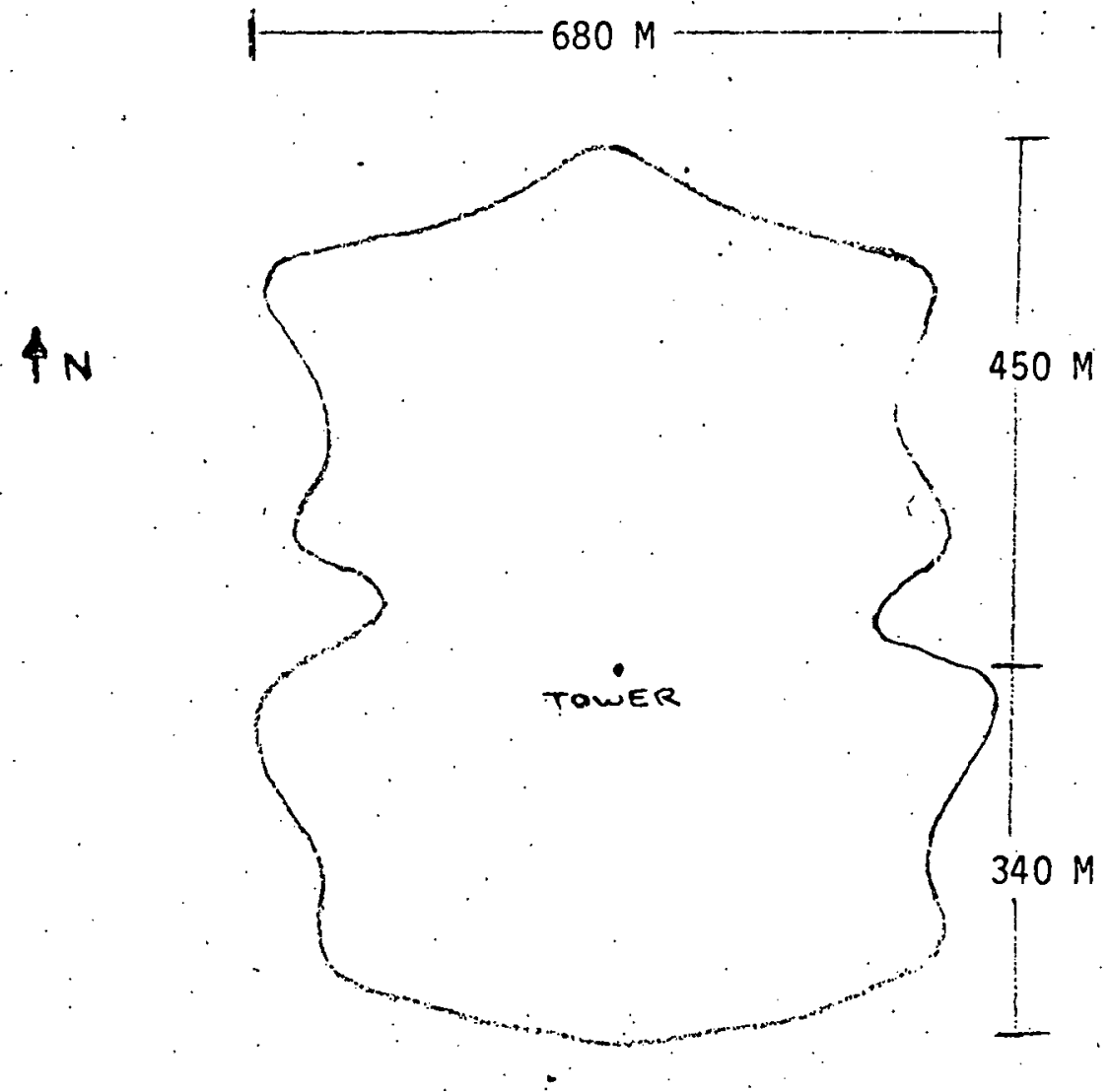

- WINTER SOLSTICE $49.9 \mathrm{MW}_{\mathrm{T}}$

- summer solstice $63.4 \mathrm{MW}_{\mathrm{T}}$

- EQUINOX $63.1 \mathrm{MW}_{\mathrm{T}}$ 


\section{METHODOLOGY}

- RUN the heliostat arRay COMPUter simulation mOdel fOR an OVERSIZED FIELD AT. WINTER SOLSTICE.

- MODIFY RESULTS TO ACCOUNT FOR DOME SHADOWING AND BLOCKING

- Select Sections of the field, in DESCENDING ORDER of EFFICIENCY, TO MEET RECEIVER THERMAL REQUIREMENT WHILE NOT VIOLATING PANEL THERMAL RESTRICTIONS.

- determine the number of heliostats in the field. And the FIELD SHAPE.

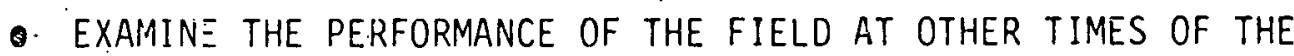
YEAR. 


\section{RECEIVER}

- CYLINDER

- HEIGHT = 17 METERS

- $\quad$ ilameter $=7$ Meters

- EFFECTIVE TARGET WIDTH $=6.67$ METERS

- TOWER HEIGHT $=95$ METERS

- THERMAL REQUIREMENT

2:00 P.M., WINTER SOLSTICE, INYOKERN

$49.9 \mathrm{MW}_{\mathrm{T}}$

- panel thermal restrictions

GREATER THAN $1: 2 \mathrm{MH}_{\mathrm{T}}$

LESS THAN

$4.2 \mathrm{MW}_{\mathrm{T}}$

- SUMMER THERMAL REQUIREMENT NOON, SUMMER SOLSTICE, INYOKERN 


\section{GROUND RULE}

- all heliostats have same dimension

- UNIFORM SPACING

- DOME DIAMETER = 7.01 METERS

- REFLECTOR RADIUS : DOME RADIUS $=.925$

- REFLECTOR DIAMETER $=6.48$ METERS

- N-S:E-W HELIOSTAT ROWS

- SPeCular reflectance $=.87$.

- specular transmittance $=.87$

- IMAgE SPREADING $=.5^{\circ}$

- FOCAL LENETH $=251$ METERS/S:N $\Psi$ $\Psi=$ TILT ANGLE FROM VERTICAL

- all heliostats aimed at taRgét center

- Aiming eRF:OR NORMALly DISTRIBUTED $\sigma=.057^{\circ}$

\section{REASON}

INTERCHANGEABILITY OF HARDWARE

SIMPLIFICATION OF ANALYSIS

MATERIAL PROPERTIES - WIND LOADING

HORIZONTAL STOW POSITION

PACKING DENSITY AND SHADOWING CONSIDERATIONS

EXPERIMENTAL DATA

EXPERIMENTAL DATA

WIDTH OF SOLAR IMAGE

GRAVITY DEFLECTION-REFLECTOR TENSION 1000 PSI

SIMPLIFICATION OF ANALYSIS 
Traverse of Reflected Image for Acquisition, Stowage.

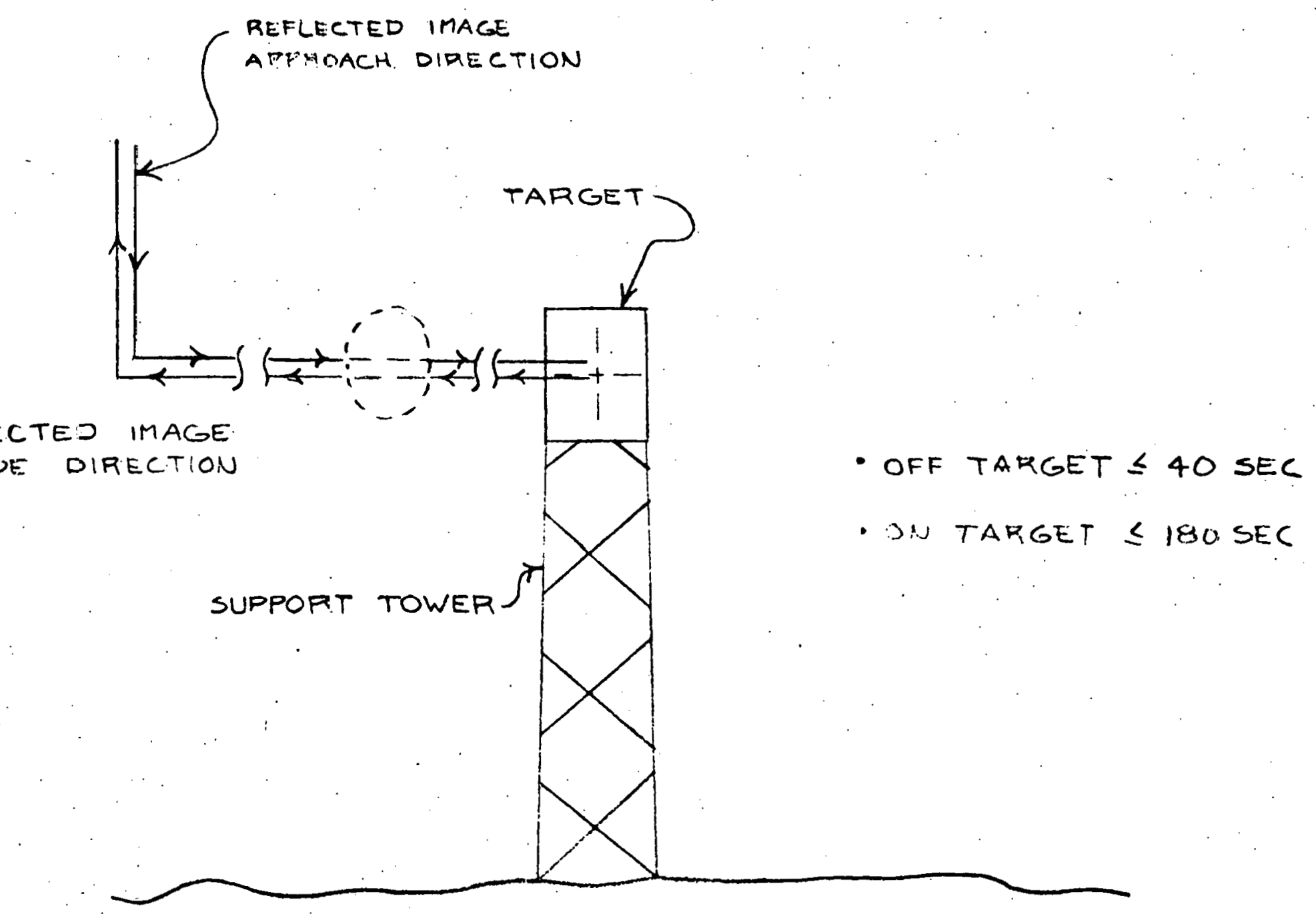

REFLECTED IMAGE RECEDE DIRECTION

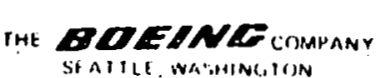


Controller Power Loss Occurrence

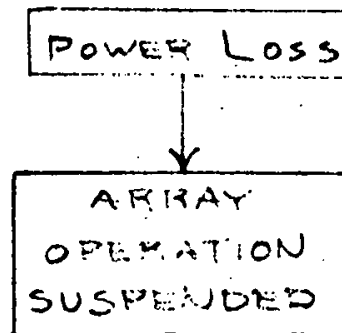

POWER ON

- automatic

- No memory loss

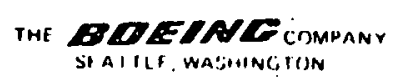


Stander Mode Costs

- INCREASED SOfTWARE PROGPAMMING I MAN-MONTH

- DECREASED STEPHEn MOTOR SIZE

- decreased STEP RE MOTOR POWER requIREMENT

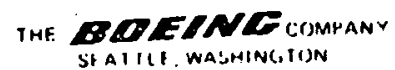


DEWPOINT VS COMPONENT TEMPERATURES winter operation at invokgran Ca.
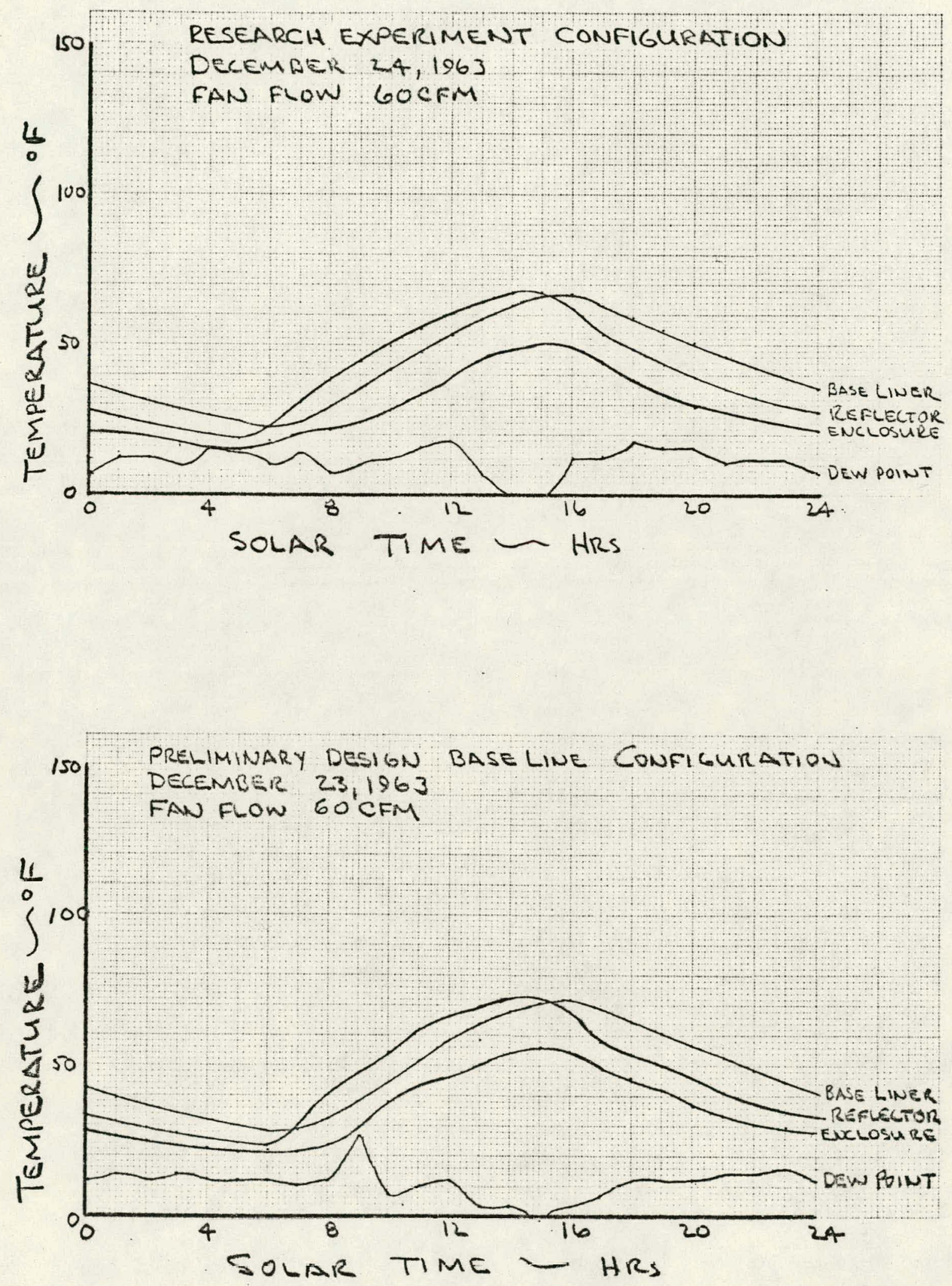


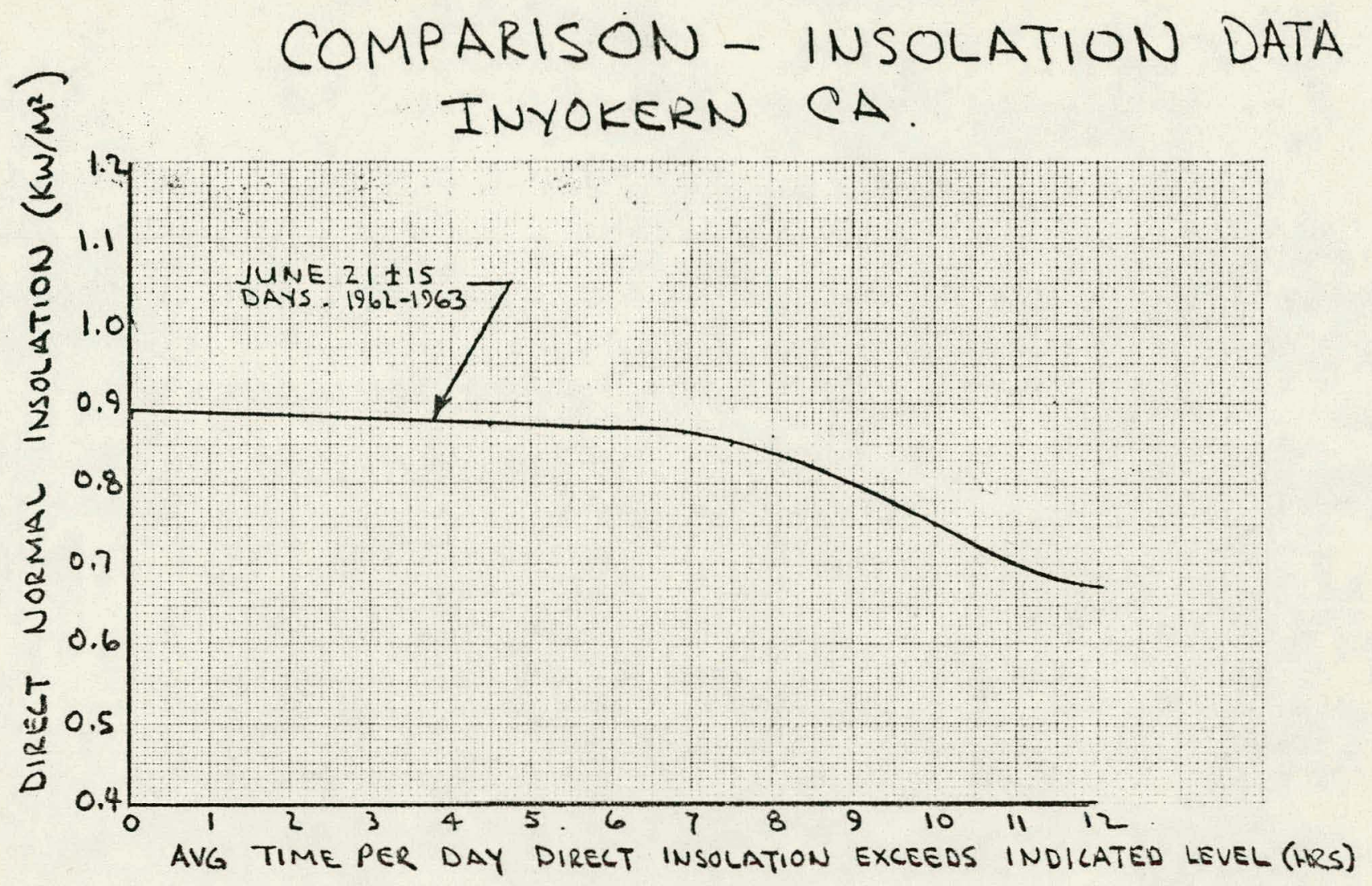

JUNE $211962-1963$ AVG - Aerospace Corp. Tape 
REFLECTOR FILM TEMPERATURE US direct solar insolation - REsearch experiment

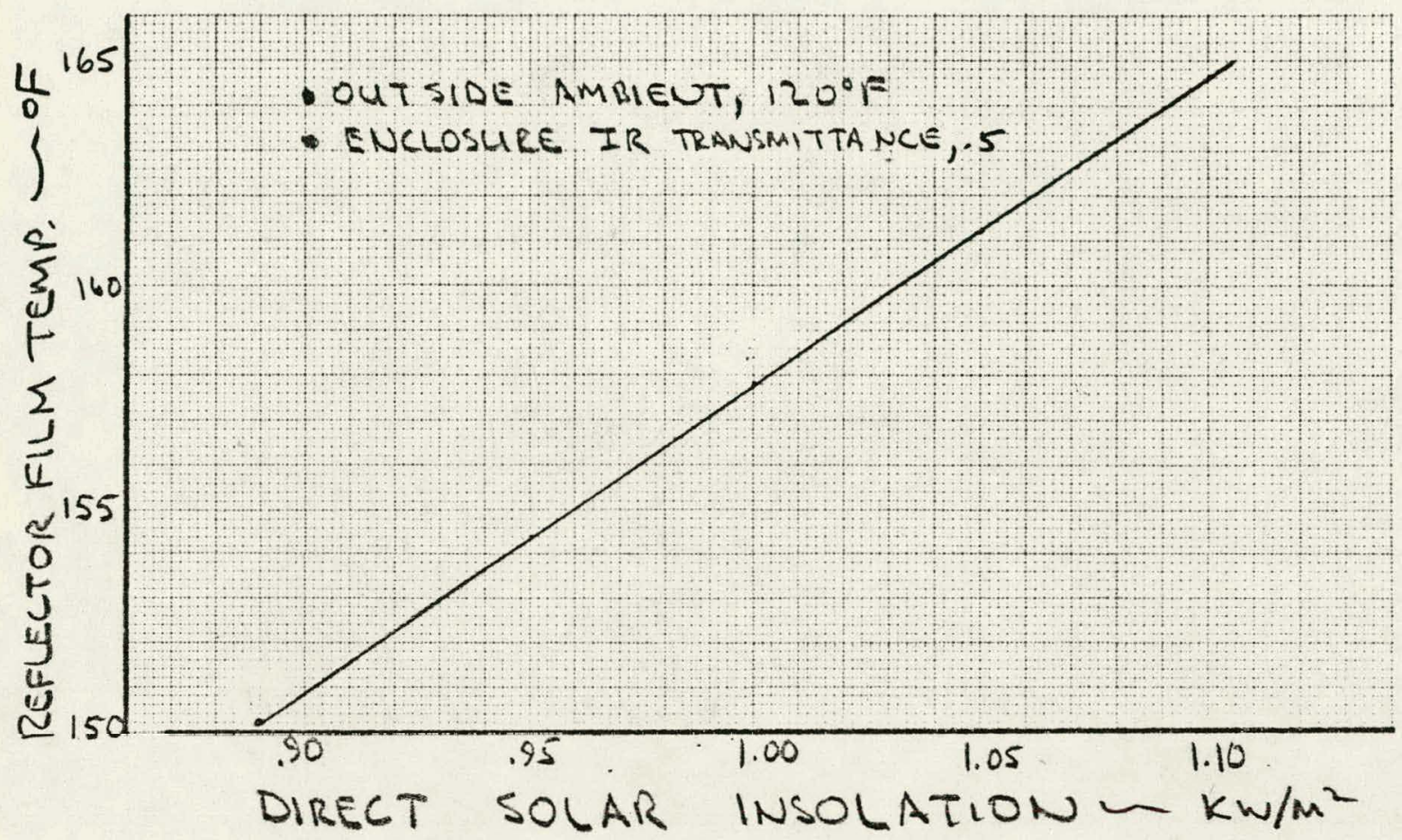

J. WATERY
Mo/1G/DS- 


\section{TELEPHOAE CALL OR YISIT RECORD}

Long Distance Telophone Call

$\square$ Local Tel ophone Coll
$\square$ Visit

FROM:

Nane J. R. Scott

Supplier

or Dept. Dupont

Phone Ho. (213) $724-4042$

City and

Stote Los Angelos, Calif.

Subject:

6 MIL FILM
P.O.

Doto February 13, 1976

Ended

Ended

Ended

TO:

Nome 0.0 . Wingrove

\section{Supplier}

or Dept.

Phone No.

City and

State

Jim Scott received the following message from Wilmington:

"Dlpont will manufacture a $6 \mathrm{mil}$ film. If it were ordared and manufactured

prior to the beginning of 1977 - it would have to be post-dried. Starting

with film produced in the early part of 1977 , the film could bo eroduced

withoult the need for post-drying. New equipment will be installed at that

time. The price for the film will be $\$ 7.50$ per $1 \mathrm{~b}$. in minimum quantities

of 5,000 lbs."

Dufont would like to know if we have any intention or desire to buy any part of

the 1,300 lbs. of film which was produced in the early January trial run. The

price; for this material will be $\$ 9.40$ per lb. in order to amortize the developmental

costs.

6.ction Required:

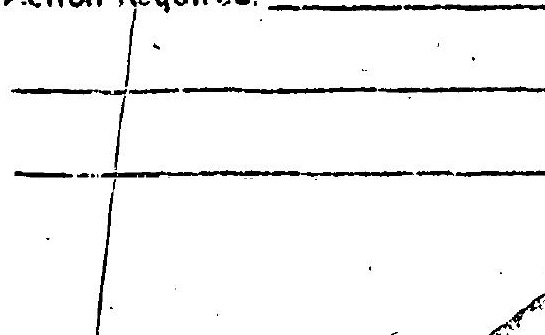

